\title{
"ENTROPIC" SOLUTIONS TO A THERMODYNAMICALLY CONSISTENT PDE SYSTEM FOR PHASE TRANSITIONS AND DAMAGE
}

\author{
ELISABETTA ROCCA AND RICCARDA ROSSI
}

\begin{abstract}
In this paper we analyze a PDE system modelling (non-isothermal) phase transitions and damage phenomena in thermoviscoelastic materials. The model is thermodynamically consistent: in particular, no small perturbation assumption is adopted, which results in the presence of quadratic terms on the right-hand side of the temperature equation, only estimated in $L^{1}$. The whole system has a highly nonlinear character.

We address the existence of a weak notion of solution, referred to as "entropic", where the temperature equation is formulated with the aid of an entropy inequality, and of a total energy inequality. This solvability concept reflects the basic principles of thermomechanics, as well as the thermodynamical consistency of the model. It allows us to obtain global-in-time existence theorems without imposing any restriction on the size of the initial data.

We prove our results by passing to the limit in a time-discretization scheme, carefully tailored to the nonlinear features of the PDE system (with its "entropic" formulation), and of the a priori estimates performed on it. Our time-discrete analysis could be useful towards the numerical study of this model.
\end{abstract}

Key words: damage, phase transitions, thermoviscoelasticity, global-in-time weak solutions, time discretization.

AMS (MOS) subject classification: 35D30, 74G25, 93C55, 82B26, 74A45.

\section{Introduction}

We consider the following PDE system

$$
\begin{aligned}
& \vartheta_{t}+\chi_{t} \vartheta+\rho \vartheta \operatorname{div}\left(\mathbf{u}_{t}\right)-\operatorname{div}(\mathrm{K}(\vartheta) \nabla \vartheta)=g+a(\chi) \varepsilon\left(\mathbf{u}_{t}\right) \mathbb{V} \varepsilon\left(\mathbf{u}_{t}\right)+\left|\chi_{t}\right|^{2} \quad \text { in } \Omega \times(0, T), \\
& \mathbf{u}_{t t}-\operatorname{div}\left(a(\chi) \mathbb{V} \varepsilon\left(\mathbf{u}_{t}\right)+b(\chi) \mathbb{E} \varepsilon(\mathbf{u})-\rho \vartheta \mathbf{1}\right)=\mathbf{f} \quad \text { in } \Omega \times(0, T), \\
& \chi_{t}+\mu \partial I_{(-\infty, 0]}\left(\chi_{t}\right)-\operatorname{div}\left(|\nabla \chi|^{p-2} \nabla \chi\right)+W^{\prime}(\chi) \ni-b^{\prime}(\chi) \frac{\varepsilon(\mathbf{u}) \mathbb{E} \varepsilon(\mathbf{u})}{2}+\vartheta \quad \text { in } \Omega \times(0, T),
\end{aligned}
$$

supplemented with the boundary conditions (here $n$ denotes the outward unit normal to $\partial \Omega$ )

$$
\mathrm{K}(\vartheta) \nabla \vartheta \cdot n=h, \quad \mathbf{u}=0, \quad \partial_{n} \chi=0 \quad \text { on } \partial \Omega \times(0, T) .
$$

Equations (1.1)-(1.3) were derived in [4] according to M. FRÉMOND's modeling approach (see [19, 20]). There, it was shown that this PDE system describes (non-isothermal) phase transitions, or (non-isothermal) damage, in a material body occupying a reference domain $\Omega \subset \mathbb{R}^{d}, d \in\{2,3\}$. We refer to [47 for a quite detailed survey on the literature on phase transition and damage problems in thermoviscoelasticity. In (1.1) - (1.3), the symbols $\vartheta$ and $\mathbf{u}$ respectively denote the absolute temperature of the system and the small displacement vector, while $\chi$ is an internal parameter: its meaning depends on the phenomenon described by (1.1)-(1.3), which also determines the choices of the coefficients $a$ and $b$ in the momentum equation (1.2), and of the constant $\mu \in\{0,1\}$ in (1.3). More precisely,

- the choices $a(\chi)=1-\chi$ and $b(\chi)=\chi$ correspond to the case of phase transitions in thermoviscoelastic materials: in this case, $\chi$ is the order parameter, standing for the local proportion of one of the two phases. We assume that $\chi$ takes values between 0 and 1 , choosing 0 and 1 as reference values: in the case of phase transitions, $\chi=1$ stands for the liquid phase while $\chi=0$ for the solid one and

Date: 19.11.2014. 
one has $0<\chi<1$ in the so-called mushy regions. Unidirectionality, or irreversibility, of the phase transition process may be encompassed in the model by taking $\mu=1$ in (1.3), which "activates" the term $\partial I_{(-\infty, 0]}\left(\chi_{t}\right)$ (i.e. the subdifferential in the sense of convex analysis of the indicator function $I_{(-\infty, 0]}$, evaluated at $\left.\chi_{t}\right)$, yielding the constraint $\chi_{t} \leq 0$ a.e. in $\Omega \times(0, T)$. The meaning of $a(\chi)=1-\chi$ and $b(\chi)=\chi$ in (1.2) is that, in the purely solid phase $\chi=0$ only the elastic energy, in addition to the thermal expansion energy, contributes to the stress $\sigma=a(\chi) \mathbb{V} \varepsilon\left(\mathbf{u}_{t}\right)+b(\chi) \mathbb{E} \varepsilon(\mathbf{u})-\rho \vartheta \mathbf{1}$ (where $\mathbb{E}$ and $\mathbb{V}$ are the elasticity and viscosity tensors, respectively). Instead, in the purely liquid, or "viscous", phase $\chi=1$ only the viscosity contribution remains, whereas in mushy regions both elastic and viscous effects are present.

- The choices $a(\chi)=b(\chi)=\chi$ correspond to damage. In this case, $\chi$ is the damage parameter, assessing the soundness of the material microscopically, around a point in the material domain $\Omega$. In fact, we have $\chi=0$ in the presence of complete damage, while $\chi$ takes the value 1 when the material is fully sound, and $0<\chi<1$ describes partial damage.

The function $\mathrm{K}$ in (1.1) is the heat conductivity, $W$ in (1.3) is a mixing energy density, which we assume of the form

$$
W=\widehat{\beta}+\widehat{\gamma} \quad \text { with } \widehat{\beta}: \operatorname{dom}(\widehat{\beta}) \rightarrow \mathbb{R} \text { convex, possibly nonsmooth, and } \widehat{\gamma} \in \mathrm{C}^{2}(\mathbb{R}),
$$

while $\mathbf{f}$ is a given bulk force, and $g$ and $h$ heat sources. The $p$-Laplacian term in (1.3) reflects the fact that we are within a gradient theory for phase transitions and damage, like in, e.g., [1, 5, 6, 19, 21, 24, 34, 37, 38, 40, where gradient regularizations are adopted in different contexts.

Observe that, in the case when both coefficients $a(\chi)$ and $b(\chi)$ in the momentum equation degenerate to zero (which happens, for instance, with $a(\chi)=b(\chi)=\chi$, when complete damage occurs), the equation for $\mathbf{u}$ looses its elliptic character. This leads to serious troubles as, for instance, no control of the term $b^{\prime}(\chi) \frac{\varepsilon(\mathbf{u}) \mathbb{E} \varepsilon(\mathbf{u})}{2}$ on the right-hand side of (1.3) is possible. That is why, in what follows we shall confine our analysis of system (1.1) - (1.3) only to the case in which the functions $a, b \in \mathrm{C}^{1}(\mathbb{R})$ are bounded from below away from 0 (cf. (2.18) in Sec. 2). We refer the reader to our previous contribution [47, where we deal with complete damage and elliptic degeneracy of the momentum equation, in a simplified case. In fact, in [47] we analyzed the following reduced system

$$
\begin{aligned}
& \vartheta_{t}+\chi_{t} \vartheta+\rho \vartheta \operatorname{div}\left(\mathbf{u}_{t}\right)-\operatorname{div}(\mathrm{K}(\vartheta) \nabla \vartheta)=g \quad \text { in } \Omega \times(0, T), \\
& \mathbf{u}_{t t}-\operatorname{div}\left(a(\chi) \mathbb{V} \varepsilon\left(\mathbf{u}_{t}\right)+b(\chi) \mathbb{E} \varepsilon(\mathbf{u})-\rho \vartheta \mathbf{1}\right)=\mathbf{f} \quad \text { in } \Omega \times(0, T), \\
& \chi_{t}+\mu \partial I_{(-\infty, 0]}\left(\chi_{t}\right)-\operatorname{div}\left(|\nabla \chi|^{p-2} \nabla \chi\right)+W^{\prime}(\chi) \ni-b^{\prime}(\chi) \frac{\varepsilon(\mathbf{u}) \mathbb{E} \varepsilon(\mathbf{u})}{2}+\vartheta \quad \text { in } \Omega \times(0, T),
\end{aligned}
$$

where the quadratic contributions in the velocities on the right-hand side in the internal energy balance (1.1) are neglected by means of the small perturbation assumption (cf. [22]).

Let us also mention that, like in [47] we confine our analysis to the case in which the thermal expansion contribution to the free energy is a linear function of the temperature $\vartheta$, and the thermal expansion coefficient $\rho$ is independent of $\chi$. The case of a $\chi$-dependent coefficient has been treated for similar PDE systems, e.g. in [5], where local-in-time results were obtained, and more recently in [26] where the existence of global-in-time weak solutions has been proved, but under the small perturbation assumption. Nonetheless, let us mention that, especially in case of phase transition phenomena, the choice of a constant $\rho$ is quite reasonable (cf., e.g., 32. for further comments on this topic).

Mathematical difficulties. In this paper, instead, we address the full system (1.1)-(1.3). Let us stress that, since we keep the quadratic terms $a(\chi) \varepsilon\left(\mathbf{u}_{t}\right) \mathbb{V} \varepsilon\left(\mathbf{u}_{t}\right)$ and $\left|\chi_{t}\right|^{2}$ on the right-hand side of (1.1), the model is thermodynamically consistent, as shown in [4]. However,

- the highly nonlinear character of the whole system, with the multivalued term $\partial I_{(-\infty, 0]}\left(\chi_{t}\right)$ and the possibly nonsmooth contribution $\widehat{\beta}$ to the energy $W$;

- the quadratic terms on the right-hand side of (1.1), which make it difficult to get suitable estimates on $(\vartheta, \mathbf{u}, \chi)$, 
bring about severe difficulties in the analysis of (1.1)-(1.3). This is the reason why we are going to develop an existence analysis only for a suitable weak solution concept for (1.1)-(1.3), which we illustrate in the following lines.

The "entropic" formulation. We resort to a weak solution notion for (1.1)-(1.3) partially mutuated from [16. There, a thermodynamically consistent model for phase transitions, consisting of the temperature and of the phase parameter equations, was analyzed: the temperature equation, featuring quadratic terms on its right-hand side, was weakly formulated in terms of an entropy inequality and of a total energy inequality. In the present framework, the pointwise internal energy balance (1.1) is thus replaced by this entropy inequality

$$
\begin{aligned}
& \int_{s}^{t} \int_{\Omega}(\log (\vartheta)+\chi) \varphi_{t} \mathrm{~d} x \mathrm{~d} r+\rho \int_{s}^{t} \int_{\Omega} \operatorname{div}\left(\mathbf{u}_{t}\right) \varphi \mathrm{d} x \mathrm{~d} r-\int_{s}^{t} \int_{\Omega} \mathrm{K}(\vartheta) \nabla \log (\vartheta) \cdot \nabla \varphi \mathrm{d} x \mathrm{~d} r \\
& \leq \int_{\Omega}(\log (\vartheta(t))+\chi(t)) \varphi(t) \mathrm{d} x-\int_{\Omega}(\log (\vartheta(s))+\chi(s)) \varphi(s) \mathrm{d} x-\int_{s}^{t} \int_{\Omega} \mathrm{K}(\vartheta) \frac{\varphi}{\vartheta} \nabla \log (\vartheta) \cdot \nabla \vartheta \mathrm{d} x \mathrm{~d} r \\
& \quad-\int_{s}^{t} \int_{\Omega}\left(g+a(\chi) \varepsilon\left(\mathbf{u}_{t}\right) \mathbb{V} \varepsilon\left(\mathbf{u}_{t}\right)+\left|\chi_{t}\right|^{2}\right) \frac{\varphi}{\vartheta} \mathrm{d} x \mathrm{~d} r-\int_{s}^{t} \int_{\partial \Omega} h \frac{\varphi}{\vartheta} \mathrm{d} S \mathrm{~d} r,
\end{aligned}
$$

where $\varphi$ is a sufficiently regular, positive test function, coupled with the following total energy inequality

$$
\mathscr{E}\left(\vartheta(t), \mathbf{u}(t), \mathbf{u}_{t}(t), \chi(t)\right) \leq \mathscr{E}\left(\vartheta(s), \mathbf{u}(s), \mathbf{u}_{t}(s), \chi(s)\right)+\int_{s}^{t} \int_{\Omega} g \mathrm{~d} x \mathrm{~d} r+\int_{0}^{t} \int_{\partial \Omega} h \mathrm{~d} S \mathrm{~d} r+\int_{s}^{t} \int_{\Omega} \mathbf{f} \cdot \mathbf{u}_{t} \mathrm{~d} x \mathrm{~d} r
$$

where

$$
\mathscr{E}\left(\vartheta, \mathbf{u}, \mathbf{u}_{t}, \chi\right):=\int_{\Omega} \vartheta \mathrm{d} x+\frac{1}{2} \int_{\Omega}\left|\mathbf{u}_{t}\right|^{2} \mathrm{~d} x+\frac{1}{2} \int_{\Omega} b(\chi(t)) \varepsilon(\mathbf{u}(t)) \mathbb{E} \varepsilon(\mathbf{u}(t)) \mathrm{d} x+\frac{1}{p} \int_{\Omega}|\nabla \chi|^{p} \mathrm{~d} x+\int_{\Omega} W(\chi) \mathrm{d} x
$$

Both (1.6) and (1.7) are, in the general case (cf. Remark 2.7later on), required to hold for almost all $t \in(0, T]$ and almost all $s \in(0, t)$, and for $s=0$. This formulation of the heat equation has been first developed in [14, 8] in the framework of heat conduction in fluids, and then applied to a phase transition model, also derived according to FRÉmonD's approach [19, firstly in [16. Successively, the so-called entropic notion of solution has been used to prove global-in-time existence results in models for special materials like liquid crystals (cf. 15, 17, 18), and more recently in the analysis of models for the evolution of non-isothermal binary incompressible immiscible fluids (cf. [13]). This notion of solution for the temperature equation corresponds exactly to the physically meaningful requirement that the system should satisfy the second and first principle of Thermodynamics. Indeed, one of the main advantages of this formulation resides in the fact that the thermodynamically consistency of the model immediately follows from the existence proof. It can be also shown that this solution concept is consistent with the standard one, (cf. the discussion in Sec. 2.3] in particular Remark 2.6, and in [16]).

From an analytical viewpoint, observe that the entropy inequality (1.6) has the advantage that all the troublesome quadratic terms on the right-hand side of (1.1) feature as multiplied by a negative test function. This, and the fact that (1.6) is an inequality, allows for upper semicontinuity arguments in the limit passage in a suitable approximation of (1.6)-(1.8).

In addition to (1.6)-(1.8), the entropic formulation of system (1.1)-(1.3) also consists of the momentum balance (1.2), given pointwise a.e. in $\Omega \times(0, T)$, and of the internal variable equation (1.3). The latter is required to hold pointwise almost everywhere in the reversible case $\mu=0$. In the irreversible case $\mu=1$, we shall confine the analysis to the case in which $\widehat{\beta}$ is the indicator function $I_{[0,+\infty)}$ of $[0,+\infty)$, hence $W(\chi)=I_{[0,+\infty)}(\chi)+\widehat{\gamma}(\chi)$. For reasons expounded in Sec. 2.3. we shall have to weakly formulate (1.3) in terms of the requirement $\chi_{t} \leq 0$ a.e. in $\Omega \times(0, T)$, of the one-sided variational inequality

$$
\int_{\Omega}\left(\chi_{t}-\operatorname{div}\left(|\nabla \chi|^{p-2} \nabla \chi\right)+\xi+\gamma(\chi)+b^{\prime}(\chi) \frac{\varepsilon(\mathbf{u}) \mathbb{E} \varepsilon(\mathbf{u})}{2}-\vartheta\right) \psi \mathrm{d} x \geq 0 \text { for all } \psi \in W^{1, p}(\Omega) \text { with } \psi \leq 0,
$$


almost everywhere in $(0, T)$ (where $\left.\gamma:=\widehat{\gamma}^{\prime}\right)$, and of the energy-dissipation inequality (for the internal variable $\chi)$

$$
\begin{aligned}
\int_{s}^{t} \int_{\Omega}\left|\chi_{t}\right|^{2} \mathrm{~d} x \mathrm{~d} r & +\int_{\Omega}\left(\frac{1}{p}|\nabla \chi(t)|^{p}+W(\chi(t))\right) \mathrm{d} x \\
& \leq \int_{\Omega}\left(\frac{1}{p}|\nabla \chi(s)|^{p}+W(\chi(s))\right) \mathrm{d} x+\int_{s}^{t} \int_{\Omega} \chi_{t}\left(-b^{\prime}(\chi) \frac{\varepsilon(\mathbf{u}) \mathbb{E} \varepsilon(\mathbf{u})}{2}+\vartheta\right) \mathrm{d} x \mathrm{~d} r
\end{aligned}
$$

for all $t \in(0, T]$ and almost all $s \in(0, t)$, with $\xi$ a selection in the (convex analysis) subdifferential $\partial \widehat{\beta}(\chi)=$ $\partial I_{[0,+\infty)}(\chi)$ of $I_{[0,+\infty)}$. In [47, Prop. 2.14] (see also [24]), we prove that, under additional regularity properties any weak solution in fact fulfills (1.3) pointwise.

Let us also mention that other approaches to the weak solvability of coupled PDE systems with an $L^{1}$-righthand side are available in the literature: in particular, we refer here to [54] and [49]. In [54], the notion of renormalized solution has been used in order to prove a global-in-time existence result for a nonlinear system in thermoviscoelasticity. In [49] the focus is on rate-independent processes coupled with viscosity and inertia in the displacement equation, and with the temperature equation. There the internal variable equation is not of gradient-flow type as (1.3), but instead features a 1-positively homogeneous dissipation potential. For the resulting PDE system, a weak solution concept partially mutuated from the theory of rate-independent processes by A. Mielke (cf., e.g., [39]) is analyzed. An existence result is proved combining techniques for rate-independent evolution, with Boccardo-Gallouët type estimates of the temperature gradient in the heat equation with $L^{1}$-right-hand side.

Our existence results. The main results of this paper, Theorems 1 and 2 , state the existence of entropic solutions for system (1.1 1.3), supplemented with the boundary conditions (1.4) (cf. Remark 2.12), in the irreversible $(\mu=1)$ and reversible $(\mu=0)$ cases.

More precisely, in the case of unidirectional evolution for $\chi$ we can prove the existence of a global-intime entropic solution (i.e. satisfying the entropy (1.6) and the total energy (1.7) inequalities, the (pointwise) momentum balance (1.2), the one-sided variational inequality (1.9) and the energy (1.10) inequalities for $\chi$ ). We work under fairly general assumptions on the nonlinear functions in (1.1)-(1.3). More precisely, we require that $a$ and $b$ are sufficiently smooth and bounded from below by a positive constant, $b$ convex, and we standardly assume that $W=I_{[0,+\infty)}+\widehat{\gamma}$, with $\widehat{\gamma}$ smooth and $\lambda$-convex. A crucial role is played by the requirement that the heat conductivity function $\mathrm{K}=\mathrm{K}(\vartheta)$ grows at least like $\vartheta^{\kappa}$ with $\kappa>1$. The reader may consult [53] for various examples in which a superquadratic growth in $\vartheta$ for the heat conductivity $\mathrm{K}$ is imposed, whereas 29 ] discusses experimental findings according to which a class of polymers exhibit a subquadratic growth for $\mathrm{K}$. Another crucial hypothesis is that the exponent $p$ in the gradient regularization of the equation for $\chi$ fulfills $p>d$. Gradient regularizations of $p$-Laplacian type, with $p>d$, have been adopted for several damage models, cf. e.g. [7, 37, 38, 30]. This, mathematically speaking, ensures that $\chi$ is estimated in $W^{1, p}(\Omega) \subset \mathrm{C}^{0}(\bar{\Omega})$. From the viewpoint of physics, since the gradient of $\chi$ accounts for interfacial energy effects in phase transitions, and for the influence of damage at a material point, undamaged in its neighborhood, in damage models, we may observe that the term $\frac{1}{p}|\nabla \chi|^{p}$ models nonlocality of the phase transition or the damage process.

Moreover, under some restriction on $\kappa$ (i.e. $\kappa \in(1,5 / 3)$ for space dimension $d=3$ ), we can also obtain an enhanced regularity for $\vartheta$ and that conclude that the total energy inequality actually holds as an equality.

In the reversible case $(\mu=0)$, instead, under the same assumptions above described (but with a general $\widehat{\beta}$ ), we improve the estimates, hence the regularity, of the internal variable $\chi$. Therefore, we prove the existence of a weak formulation of (1.1)-(1.3), featuring, in addition to (1.6), (1.7), and (1.2), a pointwise formulation of equation (1.3). Again, in the case of the aforementioned restriction on $\kappa$, we enhance the time-regularity of $\vartheta$. What is more, also exploiting the improved formulation of the equation for $\chi$, we are able to conclude existence for a stronger formulation of the heat equation (1.2), of variational type. Instead, a uniqueness result seems to be out of reach, at the moment, not only in the irreversible but also in the reversible cases (cf. Remarks 2.9 
and 2.11). Only for the isothermal reversible system a continuous dependence result, yielding uniqueness, can be proved exactly like in [47, Thm.3].

Finally, in the last Section [6] we address the analysis of system (1.1)-(1.3), with $\mu=1$, in the case the $p$-Laplacian regularization in (1.3) is replaced by the standard Laplacian operator. We approximate it by adding a $p$-Laplacian term, modulated by a small parameter $\delta$, on the left-hand side of (1.3), so that Thm. 2 , guarantees the existence of approximate solutions $\left(\vartheta_{\delta}, \mathbf{u}_{\delta}, \chi_{\delta}\right)$. Then, we let $\delta$ tend to zero. In this context, the enhanced elliptic regularity estimates on the momentum equation exploited in the proof of Thm. 1, and which would here yield some suitable compactness for the quadratic term $a\left(\chi_{\delta}\right) \varepsilon\left(\partial_{t} \mathbf{u}_{\delta}\right) \mathbb{V} \varepsilon\left(\partial_{t} \mathbf{u}_{\delta}\right)$ on the right-hand side of (1.1), are no longer available. In fact, they rely on the requirement $p>d$. A crucial step for proving the existence of (a slightly weaker notion of) entropic solutions to system (1.1)-(1.3) (cf. Theorem 6.2), then consists in deriving some suitable strong convergence for $\left(\partial_{t} \mathbf{u}_{\delta}\right)_{\delta}$ with an ad hoc technique, strongly relying on the fact that $\mu=1$, and on the additional assumption that $b$ is non-decreasing.

Our main existence results Thms. 1 and 2 are proved by passing to the limit in a time-discretization scheme, unique for the reversible and the irreversible cases, carefully tuned to the nonlinear features of the PDE system. In particular, it is devised in such a way as to obtain that the piecewise constant and piecewise linear interpolants of the discrete solutions satisfy the discrete versions of the entropy inequality (1.6), of total energy inequality (1.8), and of the energy inequality (1.10) in the case $\mu=1$. Moreover, with delicate calculations we are also able to translate on the time-discrete level a series of a priori estimates on the heat equation, having a nonlinear character. This detailed time-discrete analysis could be interesting in view of further numerical studies of this model.

For the limit passage we resort to various compactness results available in the literature, and additionally prove the compactness Theorem A.5, based on the theory of Young measures with values in infinite-dimensional (reflexive) Banach spaces.

Plan of the paper. In Section 2 we fix some notation, state some preliminaries that will be used in the rest of the paper, list our assumptions on the data as well as our main global-in-time existence results. In Section 3 we perform a series of formal a-priori estimates on our system. We render them rigorously in Section 4 , where we set up our time-discrete scheme. Theorems 11 and 2 are proved by passing to the limit in the approximated entropy and energy inequality, as well as in the discretized versions of (1.2) and (1.3), throughout Sec. 5. Section 6 is then devoted to the proof of Theorem 6.2. Finally, the Appendix contains a short recap of the theory of Young measures in infinite-dimensional Banach spaces, and the proof of Theorem A.5

\section{Setup and results}

After fixing some notation and results which shall be used throughout the paper, in Section 2.2 we collect our working assumptions on the nonlinear functions $\mathrm{K}, a, b$, and $W$ in the PDE system (1.1)-(1.3), and on the data. Then, in Secs. 2.3 and 2.4 we discuss the weak formulations of (the initial-boundary value problem for) (1.1) -(1.3) in the irreversible and reversible cases, respectively corresponding to $\mu=1$ and $\mu=0$ in (1.3).

\subsection{Preliminaries.}

Notation 2.1. Throughout the paper, given a Banach space $X$ we shall denote by $\|\cdot\|_{X}$ its norm, and use the symbol $\langle\cdot, \cdot\rangle_{X}$ for the duality pairing between $X^{\prime}$ and $X$. Moreover, we shall denote by $\mathrm{BV}([0, T] ; X)$ (by $\mathrm{C}_{\text {weak }}^{0}([0, T] ; X)$, respectively), the space of functions from $[0, T]$ with values in $X$ that are defined at every $t \in[0, T]$ and have bounded variation on $[0, T]$ (and are weakly continuous on $[0, T]$, resp.).

Let $\Omega \subset \mathbb{R}^{d}$ be a bounded domain, $d \in\{2,3\}$. We set $Q:=\Omega \times(0, T)$. We identify both $L^{2}(\Omega)$ and $L^{2}\left(\Omega ; \mathbb{R}^{d}\right)$ with their dual spaces, and denote by $(\cdot, \cdot)$ the scalar product in $\mathbb{R}^{d}$, by $(\cdot, \cdot)_{L^{2}(\Omega)}$ both the scalar product in $L^{2}(\Omega)$, and in $L^{2}\left(\Omega ; \mathbb{R}^{d}\right)$, and by $H_{0}^{1}\left(\Omega ; \mathbb{R}^{d}\right)$ and $H_{\text {Dir }}^{2}\left(\Omega ; \mathbb{R}^{d}\right)$ the spaces

$$
\begin{aligned}
& H_{0}^{1}\left(\Omega ; \mathbb{R}^{d}\right):=\left\{\mathbf{v} \in H^{1}\left(\Omega ; \mathbb{R}^{d}\right): \mathbf{v}=0 \text { on } \partial \Omega\right\}, \text { endowed with the norm }\|\mathbf{v}\|_{H_{0}^{1}\left(\Omega ; \mathbb{R}^{d}\right)}^{2}:=\int_{\Omega} \varepsilon(\mathbf{v}): \varepsilon(\mathbf{v}) \mathrm{d} x, \\
& H_{\mathrm{Dir}}^{2}\left(\Omega ; \mathbb{R}^{d}\right):=\left\{\mathbf{v} \in H^{2}\left(\Omega ; \mathbb{R}^{d}\right): \mathbf{v}=0 \text { on } \partial \Omega\right\} .
\end{aligned}
$$


Note that $\|\cdot\|_{H_{0}^{1}\left(\Omega ; \mathbb{R}^{d}\right)}$ is a norm equivalent to the standard one on $H^{1}\left(\Omega ; \mathbb{R}^{d}\right)$. We will use the symbol $\mathcal{D}(\bar{Q})$ for the space of the $C^{\infty}$-functions with compact support on $Q:=\Omega \times(0, T)$ and for $q>1$ we will adopt the notation

$$
W_{+}^{1, q}(\Omega):=\left\{\zeta \in W^{1, q}(\Omega): \zeta(x) \geq 0 \quad \text { for a.a. } x \in \Omega\right\}, \quad \text { and analogously for } W_{-}^{1, q}(\Omega) .
$$

We denote by $A_{p}$ the $p$-Laplacian operator with zero Neumann boundary conditions, viz.

$$
A_{p}: W^{1, p}(\Omega) \rightarrow W^{1, p}(\Omega)^{\prime} \text { given by }\left\langle A_{p} u, v\right\rangle_{W^{1, p}(\Omega)}:=\int_{\Omega}|\nabla u|^{p-2} \nabla u \cdot \nabla v \mathrm{~d} x .
$$

In the weak formulation of the momentum equation (1.2), besides $\mathcal{V}$ and $\mathcal{E}$ we will also make use of the operator

$$
\mathcal{C}_{\rho}: L^{2}(\Omega) \rightarrow H^{-1}\left(\Omega ; \mathbb{R}^{d}\right) \quad \text { defined by } \quad\left\langle\mathfrak{C}_{\rho}(\theta), \mathbf{v}\right\rangle_{H^{1}\left(\Omega ; \mathbb{R}^{d}\right)}:=-\rho \int_{\Omega} \theta \operatorname{div}(\mathbf{v}) \mathrm{d} x .
$$

Finally, throughout the paper we shall denote by the symbols $c, c^{\prime}, C, C^{\prime}$ various positive constants depending only on known quantities. Furthermore, the symbols $I_{i}, i=0,1, \ldots$, will be used as place-holders for several integral terms popping in the various estimates: we warn the reader that we will not be self-consistent with the numbering, so that, for instance, the symbol $I_{1}$ will occur several times with different meanings.

Recaps of mathematical elasticity. The elasticity and viscosity tensors fulfill

$$
\mathbb{E}=\left(e_{i j k h}\right), \mathbb{V}=\left(v_{i j k h}\right) \in \mathrm{C}^{1}\left(\Omega ; \mathbb{R}^{d \times d \times d \times d}\right)
$$

with coefficients satisfying the classical symmetry and ellipticity conditions (with the usual summation convention)

$$
\begin{aligned}
& e_{i j k h}=e_{j i k h}=e_{k h i j}, \quad v_{i j k h}=v_{j i k h}=v_{k h i j} \\
& \exists \alpha_{0}>0: \quad e_{i j k h} \xi_{i j} \xi_{k h} \geq \alpha_{0} \xi_{i j} \xi_{i j} \quad \forall \xi_{i j}: \xi_{i j}=\xi_{j i} \\
& \exists \beta_{0}>0: \quad v_{i j k h} \xi_{i j} \xi_{k h} \geq \beta_{0} \xi_{i j} \xi_{i j} \quad \forall \xi_{i j}: \xi_{i j}=\xi_{j i} .
\end{aligned}
$$

Observe that with (2.4) we also encompass in our analysis the case of an anisotropic and inhomogeneous material.

In order to give the variational formulation of the momentum equation, we need to introduce the bilinear forms related to the $\chi$-dependent elliptic operators appearing in (1.2). Hence, given a non-negative function $\eta \in L^{\infty}(\Omega)$ (later, $\eta=a(\chi)$ or $\left.\eta=b(\chi)\right)$, let us consider the bilinear symmetric forms $\mathrm{e}(\eta \cdot, \cdot), \mathrm{v}(\eta \cdot, \cdot)$ : $H_{0}^{1}\left(\Omega ; \mathbb{R}^{d}\right) \times H_{0}^{1}\left(\Omega ; \mathbb{R}^{d}\right) \rightarrow \mathbb{R}$ defined for all $\mathbf{u}, \mathbf{v} \in H_{0}^{1}\left(\Omega ; \mathbb{R}^{d}\right)$ by

$$
\begin{aligned}
& \mathrm{e}(\eta \mathbf{u}, \mathbf{v}):=\langle-\operatorname{div}(\eta \mathbb{E} \varepsilon(\mathbf{u})), \mathbf{v}\rangle_{H^{1}\left(\Omega ; \mathbb{R}^{d}\right)}=\sum_{i, j, k, h=1}^{d} \int_{\Omega} \eta e_{i j k h} \varepsilon_{k h}(\mathbf{u}) \varepsilon_{i j}(\mathbf{v}), \\
& \mathbf{v}(\eta \mathbf{u}, \mathbf{v}):=\langle-\operatorname{div}(\eta \mathbb{V} \varepsilon(\mathbf{u})), \mathbf{v}\rangle_{H^{1}\left(\Omega ; \mathbb{R}^{d}\right)}=\sum_{i, j, k, h=1}^{d} \int_{\Omega} \eta v_{i j k h} \varepsilon_{k h}(\mathbf{u}) \varepsilon_{i j}(\mathbf{v}) .
\end{aligned}
$$

Thanks to (2.4) and Korn's inequality (see eg [10, Thm. 6.3-3]), the forms e $(\eta \cdot, \cdot)$ and $\mathrm{v}(\eta \cdot, \cdot)$ fulfill

$$
\exists C_{1}>0 \forall \mathbf{u}, \mathbf{v} \in H_{0}^{1}\left(\Omega ; \mathbb{R}^{d}\right):\left\{\begin{array}{l}
\mathrm{e}(\eta \mathbf{u}, \mathbf{u}) \geq \inf _{x \in \Omega}(\eta(x)) C_{1}\|\mathbf{u}\|_{H^{1}(\Omega)}^{2}, \\
\mathbf{v}(\eta \mathbf{u}, \mathbf{u}) \geq \inf _{x \in \Omega}(\eta(x)) C_{1}\|\mathbf{u}\|_{H^{1}(\Omega)}^{2}
\end{array} .\right.
$$

It follows from (2.3) that they are also continuous, namely

$$
\exists C_{2}>0 \forall \mathbf{u}, \mathbf{v} \in H_{0}^{1}\left(\Omega ; \mathbb{R}^{d}\right): \quad|\mathrm{e}(\eta \mathbf{u}, \mathbf{v})|+|\mathbf{v}(\eta \mathbf{u}, \mathbf{v})| \leq C_{2}\|\eta\|_{L^{\infty}(\Omega)}\|\mathbf{u}\|_{H^{1}(\Omega)}\|\mathbf{v}\|_{H^{1}(\Omega)} .
$$

We shall denote by $\mathcal{E}(\eta \cdot): H_{0}^{1}\left(\Omega ; \mathbb{R}^{d}\right) \rightarrow H^{-1}\left(\Omega ; \mathbb{R}^{d}\right)$ and $\mathcal{V}(\eta \cdot): H_{0}^{1}\left(\Omega ; \mathbb{R}^{d}\right) \rightarrow H^{-1}\left(\Omega ; \mathbb{R}^{d}\right)$ the linear operators associated with the forms $\mathrm{e}(\eta \cdot, \cdot)$ and $\mathrm{v}(\eta \cdot, \cdot)$, respectively, that is

$$
\langle\mathcal{E}(\eta \mathbf{v}), \mathbf{w}\rangle_{H^{1}\left(\Omega ; \mathbb{R}^{d}\right)}:=\mathrm{e}(\eta \mathbf{v}, \mathbf{w}), \quad\langle\mathcal{V}(\eta \mathbf{v}), \mathbf{w}\rangle_{H^{1}\left(\Omega ; \mathbb{R}^{d}\right)}:=\mathbf{v}(\eta \mathbf{v}, \mathbf{w}) \quad \text { for all } \mathbf{v}, \mathbf{w} \in H_{0}^{1}\left(\Omega ; \mathbb{R}^{d}\right) .
$$


Remark 2.2 (A caveat on notation). Actually, it would be more appropriate to use the symbols $\mathrm{e}_{\eta}(\cdot, \cdot)$ and $\mathrm{v}_{\eta}(\cdot, \cdot)$ in place of $\mathrm{e}(\eta \cdot, \cdot), \mathrm{v}(\eta \cdot, \cdot)$ to signify that for fixed $\eta \in L^{\infty}(\Omega)$, the bilinear forms defined in (2.5) act on the pair $(\mathbf{u}, \mathbf{v})$. However, in most occurrences, we would use this notation with $\eta$ replaced by the "heavier" symbols $a(\chi)$ or $b(\chi)$. Thus, for notational simplicity we prefer to stay with the less correct notation from (2.5). The same considerations apply to the operators defined in (2.8).

It can be checked via an approximation argument that the following regularity results hold

$$
\begin{aligned}
& \text { if } \eta \in L^{\infty}(\Omega) \text { and } \mathbf{u} \in H_{0}^{1}\left(\Omega ; \mathbb{R}^{d}\right) \text {, then } \mathcal{E}(\eta \mathbf{u}), \mathcal{V}(\eta \mathbf{u}) \in H^{-1}\left(\Omega ; \mathbb{R}^{d}\right), \\
& \text { if } \eta \in W^{1, d+\epsilon}(\Omega) \text { for some } \epsilon>0 \text { and } \mathbf{u} \in H_{\mathrm{Dir}}^{2}\left(\Omega ; \mathbb{R}^{d}\right) \text {, then } \mathcal{E}(\eta \mathbf{u}), \mathcal{V}(\eta \mathbf{u}) \in L^{2}\left(\Omega ; \mathbb{R}^{d}\right) \text {. }
\end{aligned}
$$

Finally, let us recall the following elliptic regularity result, holding in the case $\Omega$ has a $\mathrm{C}^{2}$-boundary (cf. (2.15) below) and also due to (2.3), namely

$$
\exists C_{3}, C_{4}>0 \quad \forall \mathbf{u} \in H_{\operatorname{Dir}}^{2}\left(\Omega ; \mathbb{R}^{d}\right): \quad\left\{\begin{array}{l}
C_{3}\|\mathbf{u}\|_{H^{2}(\Omega)} \leq\|\operatorname{div}(\mathbb{E} \varepsilon(\mathbf{u}))\|_{L^{2}(\Omega)} \leq C_{4}\|\mathbf{u}\|_{H^{2}(\Omega)} \\
C_{3}\|\mathbf{u}\|_{H^{2}(\Omega)} \leq\|\operatorname{div}(\mathbb{V} \varepsilon(\mathbf{u}))\|_{L^{2}(\Omega)} \leq C_{4}\|\mathbf{u}\|_{H^{2}(\Omega)}
\end{array}\right.
$$

For this, we refer e.g. [41, Lemma 3.2, p. 260]) or [27, Chap. 6, p. 318].

Useful inequalities. In order to make the paper as self-contained as possible, we recall here the GagliardoNirenberg inequality (cf. [42, p. 125]) in a particular case: for all $r, q \in[1,+\infty]$, and for all $v \in L^{q}(\Omega)$ such that $\nabla v \in L^{r}(\Omega)$, there holds

$$
\|v\|_{L^{s}(\Omega)} \leq C_{\mathrm{GN}}\|v\|_{W^{1, r}(\Omega)}^{\theta}\|v\|_{L^{q}(\Omega)}^{1-\theta} \quad \text { with } \frac{1}{s}=\theta\left(\frac{1}{r}-\frac{1}{d}\right)+(1-\theta) \frac{1}{q}, \quad 0 \leq \theta \leq 1,
$$

the positive constant $C_{\mathrm{GN}}$ depending only on $d, r, q, \theta$. Combining the compact embedding

$$
H_{\text {Dir }}^{2}\left(\Omega ; \mathbb{R}^{d}\right) \Subset W^{1, d^{\star}-\eta}\left(\Omega ; \mathbb{R}^{d}\right), \quad \text { with } d^{\star}=\left\{\begin{array}{ll}
\infty & \text { if } d=2, \\
6 & \text { if } d=3,
\end{array} \text { for all } \eta>0,\right.
$$

(where for $d=2$ we mean that $H_{\text {Dir }}^{2}\left(\Omega ; \mathbb{R}^{d}\right) \Subset W^{1, q}\left(\Omega ; \mathbb{R}^{d}\right)$ for all $1 \leq q<\infty$ ), with [33, Thm. 16.4, p. 102], we have

$$
\forall \varrho>0 \quad \exists C_{\varrho}>0 \quad \forall \mathbf{u} \in H_{\text {Dir }}^{2}\left(\Omega ; \mathbb{R}^{d}\right):\|\varepsilon(\mathbf{u})\|_{L^{d^{\star}-\eta}(\Omega)} \leq \varrho\|\mathbf{u}\|_{H^{2}(\Omega)}+C_{\varrho}\|\mathbf{u}\|_{L^{2}(\Omega)} .
$$

We will also use the following nonlinear Poincaré-type inequality (cf. e.g. [23, Lemma 2.2]), with $m(w)$ the mean value of $w$ :

$$
\forall q>0 \quad \exists C_{q}>0 \quad \forall w \in H^{1}(\Omega): \quad\left\||w|^{q} w\right\|_{H^{1}(\Omega)} \leq C_{q}\left(\left\|\nabla\left(|w|^{q} w\right)\right\|_{L^{2}(\Omega)}+|m(w)|^{q+1}\right) .
$$

2.2. Assumptions. In most of this paper, we shall work under the following

Hypothesis (0). We suppose that

$$
\Omega \subset \mathbb{R}^{d}, \quad d \in\{2,3\} \text { is a bounded connected domain, with } \mathrm{C}^{2} \text {-boundary } \partial \Omega
$$

and that the viscosity tensor is given by

$$
\mathbb{V}=\omega \mathbb{E} \quad \text { for a constant } \omega>0 .
$$

Remark 2.3. The smoothness requirement (2.15) will allow us to apply the elliptic regularity results in (2.10).

Concerning (2.16), let us mention in advance that it will only be used in the proof of the $H^{2}\left(\Omega ; \mathbb{R}^{d}\right)$-regularity for the discrete displacements, cf. Lemma 4.4 ahead ensuring the existence of solutions to the time-discretization scheme for system (1.1)-(1.3). As we will see, this regularity property is crucial for the rigorous proof of the elliptic regularity estimate for the displacements, see the Fifth estimate (formally) derived in Sec. 3, which is in turn essential in the proof of our main results, Theorems 1 and 2 ,

Instead, in the proof of Thm.6.2 we will not need to perform the aforementioned elliptic regularity argument, at the price of proving the existence of a weaker notion of solution for the irreversible system (cf. (6.5) and Remark6.3 in Sec. 6). Hence, we will be able to dispense with conditions (2.15) and (2.16). This is the reason 
why, despite $\mathbb{V}$ and $\mathbb{E}$ are a multiple of each other by (2.16), we have kept the two symbols $\mathbb{V}$ and $\mathbb{E}$ throughout the paper.

We list below our basic assumptions on the functions $\mathrm{K}, a, b$, and $W$ in system (1.1)-(1.3).

Hypothesis (I). We suppose that

$$
\begin{aligned}
& \text { the function } \mathrm{K}:[0,+\infty) \rightarrow(0,+\infty) \text { is continuous and } \\
& \exists c_{0}, c_{1}>0 \quad \kappa>1 \quad \forall \vartheta \in[0,+\infty): \quad c_{0}\left(1+\vartheta^{\kappa}\right) \leq \mathrm{K}(\vartheta) \leq c_{1}\left(1+\vartheta^{\kappa}\right) .
\end{aligned}
$$

We will denote by $\widehat{\mathrm{K}}$ the primitive $\widehat{\mathrm{K}}(x):=\int_{0}^{x} \mathrm{~K}(r) \mathrm{d} r$ of $\mathrm{K}$.

Hypothesis (II). We require

$$
a \in \mathrm{C}^{1}(\mathbb{R}), b \in \mathrm{C}^{2}(\mathbb{R}) \text { and } \exists c_{2}>0: \quad a(x), b(x) \geq c_{2} \text { for all } x \in \mathbb{R}
$$

and that the function $b$ is convex. The latter requirement could be weakened to $\lambda$-convexity, i.e. that $b^{\prime \prime}$ is bounded from below (cf. also (2.21)), see. Remark 4.9 later on.

Hypothesis (III). We suppose that the potential $W$ in (1.3) is given by $W=\widehat{\beta}+\widehat{\gamma}$, where

$$
\begin{aligned}
& \widehat{\beta}: \mathbb{R} \rightarrow \mathbb{R} \cup\{+\infty\} \text { has nonempty domain } \operatorname{dom}(\widehat{\beta}), \text { is l.s.c. and convex, } \widehat{\gamma} \in \mathrm{C}^{2}(\mathbb{R}), \\
& \exists c_{W} \in \mathbb{R} \quad W(r) \geq c_{W} \quad \forall r \in \operatorname{dom}(\widehat{\beta}) .
\end{aligned}
$$

Moreover, we impose that

$$
\exists \lambda>0 \quad \forall r \in \mathbb{R}: \quad \widehat{\gamma}^{\prime \prime}(r) \geq-\lambda .
$$

Hereafter, we shall use the notation

$$
\beta:=\partial \widehat{\beta}, \quad \gamma:=\widehat{\gamma}^{\prime} .
$$

Observe that, we have not required that $\operatorname{dom}(\widehat{\beta}) \subset[0,+\infty)$, which would enforce the (physically feasible) positivity of the phase/damage variable $\chi$. In fact, for the analysis of the irreversible case (i.e. with $\mu=1$ ), we will have to confine the discussion to the case $\widehat{\beta}=I_{[0,+\infty)}$, cf. Hypothesis (IV) later on. Instead, in the reversible case $\mu=0$, we will allow for a general $\widehat{\beta}$ (complying with Hypothesis (III)).

Remark 2.4 (A generalization of the $p$-Laplacian). In fact, our analysis of system (1.1)-(1.3) extends to the case that the $p$-Laplacian operator $-\operatorname{div}\left(|\nabla \chi|^{p-2} \nabla \chi\right)$, with $p>d$, is replaced by an elliptic operator $\mathcal{B}: W^{1, p}(\Omega) \rightarrow W^{1, p}(\Omega)^{*}$ of the form

$$
\langle\mathcal{B}(\chi), v\rangle_{W^{1, p}(\Omega)}:=\int_{\Omega} \nabla_{\zeta} \phi(x, \nabla \chi(x)) \cdot \nabla v(x) \mathrm{d} x,
$$

where $\phi: \Omega \times \mathbb{R}^{d} \rightarrow[0,+\infty)$ is a Carathéodory integrand such that

$$
\begin{aligned}
& \text { the map } \phi(x, \cdot): \mathbb{R}^{d} \rightarrow[0,+\infty) \text { is convex, with } \phi(x, 0)=0 \text {, and in } \mathrm{C}^{1}\left(\mathbb{R}^{d}\right) \text { for a.a. } x \in \Omega, \\
& \exists c_{3}, c_{4}, c_{5}>0 \text { for a.a. } x \in \Omega \quad \forall \boldsymbol{\zeta} \in \mathbb{R}^{d}:\left\{\begin{array}{l}
\phi(x, \boldsymbol{\zeta}) \geq c_{3}|\boldsymbol{\zeta}|^{p}-c_{4}, \\
\left|\nabla_{\zeta} \phi(x, \boldsymbol{\zeta})\right| \leq c_{5}\left(1+|\zeta|^{p-1}\right) .
\end{array}\right.
\end{aligned}
$$

This more general framework was analyzed in [4], to which we refer the reader for all details.

Problem and Cauchy data. We suppose that the data $\mathbf{f}, g$, and $h$ fulfill

$$
\begin{aligned}
& \mathbf{f} \in L^{2}\left(0, T ; L^{2}\left(\Omega ; \mathbb{R}^{d}\right)\right), \\
& g \in L^{1}\left(0, T ; L^{1}(\Omega)\right) \cap L^{2}\left(0, T ; H^{1}(\Omega)^{\prime}\right), \quad g \geq 0 \quad \text { a.e. in } \Omega \times(0, T), \\
& h \in L^{1}\left(0, T ; L^{2}(\partial \Omega)\right), \quad h \geq 0 \quad \text { a.e. in } \partial \Omega \times(0, T),
\end{aligned}
$$


and that the initial data comply with

$$
\begin{aligned}
& \vartheta_{0} \in L^{1}(\Omega), \quad \exists \vartheta_{*}>0: \quad \inf _{\Omega} \vartheta_{0} \geq \vartheta_{*}>0, \quad \log \vartheta_{0} \in L^{1}(\Omega), \\
& \mathbf{u}_{0} \in H_{\text {Dir }}^{2}\left(\Omega ; \mathbb{R}^{d}\right), \quad \mathbf{v}_{0} \in H_{0}^{1}\left(\Omega ; \mathbb{R}^{d}\right), \\
& \chi_{0} \in W^{1, p}(\Omega), \quad \widehat{\beta}\left(\chi_{0}\right) \in L^{1}(\Omega) .
\end{aligned}
$$

Let us mention in advance that the strict positivity requirement on $\vartheta_{0}$ and the non-negativity of $g$ and $h$ serve to the purpose of ensuring the existence of an entropic solution $(\vartheta, \mathbf{u}, \chi)$ to (the initial-boundary value problem for system) (1.1)-(1.3), with $\vartheta$ strictly positive. The latter property has a crucial physical meaning, as $\vartheta$ is the absolute temperature of the system. It also underlies our notion of weak solution for the heat equation, involving the term $\log (\vartheta)$.

2.3. A global existence result for the irreversible system. Before stating precisely our notion of weak solution to (the initial-boundary value problem for) system (1.1)-1.3) in the case of unidirectional evolution, let us briefly motivate the weak formulations for the heat balance equation (1.1), and for the phase/damage parameter subdifferential inclusion (1.3) (with $\mu=1$ ). They will be coupled with the pointwise (in time and space) formulation of the momentum equation (1.2) (cf. (2.42) later on).

Entropy and total energy inequalities for the heat equation. For (1.1), we adopt the weak formulation of proposed in [8, 14, 16. It consists of a so-called "entropy inequality", and of a "total energy (in)equality". The former is obtained by formally dividing (1.1) by $\vartheta$, and testing it by a smooth test function $\varphi$. Integrating over space and time leads to

$$
\begin{aligned}
\int_{0}^{T} \int_{\Omega}\left(\partial_{t} \log (\vartheta)+\chi_{t}\right. & \left.+\rho \operatorname{div}\left(\mathbf{u}_{t}\right)\right) \varphi \mathrm{d} x \mathrm{~d} t+\int_{0}^{T} \int_{\Omega} \mathrm{K}(\vartheta) \nabla \log (\vartheta) \nabla \varphi \mathrm{d} x \mathrm{~d} t \\
& -\int_{0}^{T} \int_{\Omega} \mathrm{K}(\vartheta) \frac{\varphi}{\vartheta} \nabla \log (\vartheta) \nabla \vartheta \mathrm{d} x \mathrm{~d} t \\
& =\int_{0}^{T} \int_{\Omega}\left(g+a(\chi) \varepsilon\left(\mathbf{u}_{t}\right) \mathbb{V} \varepsilon\left(\mathbf{u}_{t}\right)+\left|\chi_{t}\right|^{2}\right) \frac{\varphi}{\vartheta} \mathrm{d} x \mathrm{~d} t+\int_{0}^{T} \int_{\partial \Omega} h \frac{\varphi}{\vartheta} \mathrm{d} S \mathrm{~d} t
\end{aligned}
$$

for all $\varphi \in \mathcal{D}(\bar{Q})$. Then, the entropy inequality (2.39) later on follows. The total energy inequality (2.40) associated with system (1.1)-(1.3) is obtained by testing (1.1) by 1 , (1.2) by $\mathbf{u}_{t}$, and (1.3) by $\chi_{t}$.

Let us mention in advance that the entropy inequality (2.39) below has the advantage that all the troublesome quadratic quantities on the right-hand side of (1.1) are tested by the negative function $-\varphi$. This will allow for upper semicontinuity arguments in the limit passage for proving the existence of weak solutions, cf. Sec. 5 later on. Let us also mention in advance that, when dropping the unidirectionality constraint (i.e., in the case $\mu=0$ ), under an additional condition (cf. Hypothesis (V)), we will be able to get an existence result for an improved formulation of (1.1), cf. Theorem 2 below.

Weak formulation of the flow rule for $\chi$. A significant difficulty in the analysis of system (1.1)-(1.3) is due to the triply nonlinear character of (1.3), featuring, in addition to the $p$-Laplacian and to $\beta=\partial \widehat{\beta}$ which contributes to $W^{\prime}$, the (maximal monotone) operator $\partial I_{(-\infty, 0]}$. Since the latter is unbounded, it is not possible to perform comparison estimates in (1.3) and an estimate for the terms $A_{p} \chi$ and $\beta(\chi)$ (treated as single-valued in the context of this heuristic discussion) could be obtained only by testing (1.3) by $\partial_{t}\left(A_{p} \chi+\beta(\chi)\right)$. However, the related calculations, involving an integration by parts in time on the right-hand side of (1.3), cannot be carried out in the present case. That is why, we need to resort to a weak formulation of (1.3) which does not feature the term $A_{p} \chi+\beta(\chi)$. We draw it from [24, 25, and as therein we confine the analysis to the particular case in which

\section{Hypothesis (IV).}

This still ensures the constraint

$$
\widehat{\beta}=I_{[0,+\infty)} .
$$

$$
\chi \in[0,1] \quad \text { a.e. in } \Omega \times(0, T)
$$


provided we start from an initial datum $\chi_{0} \leq 1$ a.e. in $\Omega$, we will obtain by irreversibility that $\chi(t) \leq \chi_{0} \leq 1$ a.e. in $\Omega$, for almost all $t \in(0, T)$.

To motivate the weak formulation of (1.3) from [24, 25, we observe that (1.3) rephrases as

$$
\begin{aligned}
& \chi_{t} \leq 0 \quad \text { in } \Omega \times(0, T), \\
& \left(\chi_{t}-\operatorname{div}\left(|\nabla \chi|^{p-2} \nabla \chi\right)+\xi+\gamma(\chi)+b^{\prime}(\chi) \frac{\varepsilon(\mathbf{u}) \mathbb{E} \varepsilon(\mathbf{u})}{2}-\vartheta\right) \psi \geq 0 \text { for all } \psi \leq 0 \quad \text { in } \Omega \times(0, T), \\
& \left(\chi_{t}-\operatorname{div}\left(|\nabla \chi|^{p-2} \nabla \chi\right)+\xi+\gamma(\chi)+b^{\prime}(\chi) \frac{\varepsilon(\mathbf{u}) \mathbb{E} \varepsilon(\mathbf{u})}{2}-\vartheta\right) \chi_{t} \leq 0 \quad \text { in } \Omega \times(0, T),
\end{aligned}
$$

with $\xi \in \partial I_{[0,+\infty)}(\chi)$ in $\Omega \times(0, T)$. Our weak formulation of (1.3) in fact consists of (2.32a), of the integrated version of (2.32b), with negative test functions from $W^{1, p}(\Omega)$, and of the energy inequality obtained by integrating (2.32c). In [47, Prop. 2.14] (see also [24, Thm. 4.6]), we prove that, under additional regularity properties, any weak solution in the sense of (2.43)-(2.46) in fact fulfills (1.3) pointwise.

We are now in the position to specify our weak solution concept, for which we borrow the terminology from [16].

Definition 2.5 (Entropic solutions to the irreversible system). Let $\mu=1$. Given initial data $\left(\vartheta_{0}, \mathbf{u}_{0}, \mathbf{v}_{0}, \chi_{0}\right)$ fulfilling (2.26) -(2.28), we call a triple $(\vartheta, \mathbf{u}, \chi)$ an entropic solution to the (initial-boundary value problem) for system (1.1)-(1.3), with the boundary conditions (1.4), if

$$
\begin{aligned}
& \vartheta \in L^{2}\left(0, T ; H^{1}(\Omega)\right) \cap L^{\infty}\left(0, T ; L^{1}(\Omega)\right), \\
& \log (\vartheta) \in L^{2}\left(0, T ; H^{1}(\Omega)\right), \\
& \mathbf{u} \in H^{1}\left(0, T ; H_{\text {Dir }}^{2}\left(\Omega ; \mathbb{R}^{d}\right)\right) \cap W^{1, \infty}\left(0, T ; H_{0}^{1}\left(\Omega ; \mathbb{R}^{d}\right)\right) \cap H^{2}\left(0, T ; L^{2}\left(\Omega ; \mathbb{R}^{d}\right)\right), \\
& \chi \in L^{\infty}\left(0, T ; W^{1, p}(\Omega)\right) \cap H^{1}\left(0, T ; L^{2}(\Omega)\right),
\end{aligned}
$$

$(\vartheta, \mathbf{u}, \chi)$ complies with the initial conditions

$$
\begin{array}{ll}
\mathbf{u}(0, x)=\mathbf{u}_{0}(x), \quad \mathbf{u}_{t}(0, x)=\mathbf{v}_{0}(x) & \text { for a.a. } x \in \Omega, \\
\chi(0, x)=\chi_{0}(x) & \text { for a.a. } x \in \Omega,
\end{array}
$$

(while the initial condition for $\vartheta$ is implicitly formulated in (2.40) below), and with the entropic formulation of (1.1)-(1.3), consisting of

- the entropy inequality for almost all $t \in(0, T]$ and almost all $s \in(0, t)$, and for $s=0$ :

$$
\begin{aligned}
& \int_{s}^{t} \int_{\Omega}(\log (\vartheta)+\chi) \varphi_{t} \mathrm{~d} x \mathrm{~d} r-\rho \int_{s}^{t} \int_{\Omega} \operatorname{div}\left(\mathbf{u}_{t}\right) \varphi \mathrm{d} x \mathrm{~d} r-\int_{s}^{t} \int_{\Omega} \mathrm{K}(\vartheta) \nabla \log (\vartheta) \cdot \nabla \varphi \mathrm{d} x \mathrm{~d} r \\
& \leq \int_{\Omega}(\log (\vartheta(t))+\chi(t)) \varphi(t) \mathrm{d} x-\int_{\Omega}(\log (\vartheta(s))+\chi(s)) \varphi(s) \mathrm{d} x-\int_{s}^{t} \int_{\Omega} \mathrm{K}(\vartheta) \frac{\varphi}{\vartheta} \nabla \log (\vartheta) \cdot \nabla \vartheta \mathrm{d} x \mathrm{~d} r \\
& -\int_{s}^{t} \int_{\Omega}\left(g+a(\chi) \varepsilon\left(\mathbf{u}_{t}\right) \mathbb{V} \varepsilon\left(\mathbf{u}_{t}\right)+\left|\chi_{t}\right|^{2}\right) \frac{\varphi}{\vartheta} \mathrm{d} x \mathrm{~d} r-\int_{s}^{t} \int_{\partial \Omega} h \frac{\varphi}{\vartheta} \mathrm{d} S \mathrm{~d} r
\end{aligned}
$$

for all $\varphi$ in $\mathrm{C}^{0}\left([0, T] ; W^{1, d+\epsilon}(\Omega)\right)$ for some $\epsilon>0$, and $\varphi \in H^{1}\left(0, T ; L^{6 / 5}(\Omega)\right)$, with $\varphi \geq 0$;

- the total energy inequality for almost all $t \in(0, T]$ and almost all $s \in(0, t)$, and for $s=0$ :

$$
\mathscr{E}\left(\vartheta(t), \mathbf{u}(t), \mathbf{u}_{t}(t), \chi(t)\right) \leq \mathscr{E}\left(\vartheta(s), \mathbf{u}(s), \mathbf{u}_{t}(s), \chi(s)\right)+\int_{s}^{t} \int_{\Omega} g \mathrm{~d} x \mathrm{~d} r+\int_{s}^{t} \int_{\partial \Omega} h \mathrm{~d} S \mathrm{~d} r+\int_{s}^{t} \int_{\Omega} \mathbf{f} \cdot \mathbf{u}_{t} \mathrm{~d} x \mathrm{~d} r,
$$

where for $s=0$ we read $\vartheta_{0}$, and

$$
\mathscr{E}\left(\vartheta, \mathbf{u}, \mathbf{u}_{t}, \chi\right):=\int_{\Omega} \vartheta \mathrm{d} x+\frac{1}{2} \int_{\Omega}\left|\mathbf{u}_{t}\right|^{2} \mathrm{~d} x+\frac{1}{2} \mathrm{e}(b(\chi) \mathbf{u}, \mathbf{u})+\frac{1}{p} \int_{\Omega}|\nabla \chi|^{p} \mathrm{~d} x+\int_{\Omega} W(\chi) \mathrm{d} x ;
$$


- the momentum equation

$$
\mathbf{u}_{t t}+\mathcal{V}\left(a(\chi) \mathbf{u}_{t}\right)+\mathcal{E}(b(\chi) \mathbf{u})+\mathcal{C}_{\rho}(\vartheta)=\mathbf{f} \quad \text { a.e. in } \Omega \times(0, T) ;
$$

- the weak formulation of (1.3), viz.

$$
\begin{aligned}
& \chi_{t}(x, t) \leq 0 \quad \text { for a.a. }(x, t) \in \Omega \times(0, T), \\
& \int_{\Omega}\left(\chi_{t}(t) \psi+|\nabla \chi(t)|^{p-2} \nabla \chi(t) \cdot \nabla \psi+\xi(t) \psi+\gamma(\chi(t)) \psi+b^{\prime}(\chi(t)) \frac{\varepsilon(\mathbf{u}(t)) \mathbb{E} \varepsilon(\mathbf{u}(t))}{2} \psi-\vartheta(t) \psi\right) \mathrm{d} x \geq 0 \\
& \quad \text { for all } \psi \in W_{-}^{1, p}(\Omega), \quad \text { for a.a.t } \in(0, T),
\end{aligned}
$$

where $\xi \in \partial I_{[0,+\infty)}(\chi)$ in the sense that

$\xi \in L^{1}\left(0, T ; L^{1}(\Omega)\right) \quad$ and $\quad\langle\xi(t), \psi-\chi(t)\rangle_{W^{1, p}(\Omega)} \leq 0 \quad \forall \psi \in W_{+}^{1, p}(\Omega)$, for a.a.t $\in(0, T)$,

as well as the energy-dissipation inequality (for $\chi$ ) for all $t \in(0, T]$, for $s=0$, and for almost all $0<s \leq t$

$$
\begin{aligned}
\int_{s}^{t} \int_{\Omega}\left|\chi_{t}\right|^{2} \mathrm{~d} x \mathrm{~d} r & +\int_{\Omega}\left(\frac{1}{p}|\nabla \chi(t)|^{p}+W(\chi(t))\right) \mathrm{d} x \\
& \leq \int_{\Omega}\left(\frac{1}{p}|\nabla \chi(s)|^{p}+W(\chi(s))\right) \mathrm{d} x+\int_{s}^{t} \int_{\Omega} \chi_{t}\left(-b^{\prime}(\chi) \frac{\varepsilon(\mathbf{u}) \mathbb{E} \varepsilon(\mathbf{u})}{2}+\vartheta\right) \mathrm{d} x \mathrm{~d} r .
\end{aligned}
$$

Remark 2.6 (Consistency of the entropic and the classical formulations of (1.1)). It can be checked that, in case the functions $\vartheta$ and $\chi$ are sufficiently smooth, inequalities (2.39)-(2.40), combined with (1.2) and (1.3), yield the (pointwise formulation of the) heat equation (1.1).

To check this, by contradiction suppose that (the weak formulation of) (1.1) does not hold. Since (1.1) is equivalent to (2.39) with identity sign, we then would have that (2.39) holds with a strict inequality sign. Hence, we could test (1.2) by $\mathbf{u}_{t}$, (1.3) by $\chi_{t}$, and choose $\varphi=\vartheta$ (which is admissible for a sufficiently smooth $\vartheta$ ) in (2.39) (with a strict inequality). Summing up the relations thus obtained, we would conclude the total energy balance (2.40) is not satisfied.

However, at the moment the necessary regularity for $\vartheta$ and $\chi$ to carry out this argument is out of reach.

Remark 2.7 (Validity of the total energy inequality). Let us mention here that originally in [14, 8] the total energy inequality (2.40) was required to be hold as an equality on every sub-interval $(s, t) \subset[0, T]$. However, in the present setting, in general we will be able to obtain it only as an inequality on $(s, t)$ for almost all $s, t \in(0, T)$.

Indeed, we will prove (2.40) by passing to the limit in its time-discrete version, involving an approximate total energy functional evaluated at approximate solutions. Due to the lack of suitable estimates on the latter sequences, and to the presence of nonlinear and nonsmooth terms in the energy (related to the high order and non-smooth nonlinearities in the $\chi$-equation (1.3) $)$, we will be able to prove the pointwise convergence of the approximate total energy functional only almost everywhere on $(0, T)$.

Yet, in the irreversibile case $\mu=1$ we will slightly improve (2.40) under a further condition on $\mathrm{K}$ (see Thm. 11). We will considerably enhance it in the reversible case $\mu=0$ and under suitable growth conditions on the heat conductivity K (cf. Thm. 2).

Remark 2.8 (Total energy inequality and energy-dissipation inequality for $\chi$ ). As already pointed out, the total energy inequality (2.40) (formally) results from testing the heat equation by 1 , the momentum equation by $\mathbf{u}_{t}$, the flow rule for $\chi$ by $\chi_{t}$, and integrating in time. The latter test also gives rise to the energy-dissipation inequality (2.46).

However, let us stress that, in the present setting, (2.40) and (2.46) cannot be obtained one from another. Indeed, to do so, it would be necessary to test the entropy inequality by $\vartheta$ (which would correspond to testing the heat equation by 1), which is not an admissible test function for (2.39) due to its low regularity (2.33). 
We now state our existence result for system (1.1)-(1.3) in the case $\mu=1$. As far as the time-regularity of $\vartheta$ goes, observe that we will just prove BV-in-time regularity for $\log (\vartheta)$ (cf. (2.48) below). Indeed, we will obtain BV-in-time regularity for $\vartheta$, as well, under an additional restriction on the exponent $\kappa$ in Hypothesis (I) (note that the range of the admissible values below depends on the space dimension), viz.

Hypothesis (V). The exponent $\kappa$ in (2.17) satisfies

$$
\kappa \in(1,5 / 3) \quad \text { if } d=3 \text { and } \kappa \in(1,2) \quad \text { if } d=2 .
$$

Theorem 1 (Existence of entropic solutions, $\mu=1$ ). Let $\mu=1$. Assume Hypotheses (0)-(III) and, in addition, (IV) (i.e., $\widehat{\beta}=I_{[0,+\infty)}$ ), as well as conditions (2.23) $(2.28)$ on the data $\mathbf{f}, g, h, \vartheta_{0}, \mathbf{u}_{0}, \mathbf{v}_{0}, \chi_{0}$. Then, there exists an entropic solution (in the sense of Definition 2.5) $(\vartheta, \mathbf{u}, \chi)$ to the initial-boundary value problem for system (1.1)-(1.3), such that

$$
\log (\vartheta) \in L^{\infty}\left(0, T ; W^{1, d+\epsilon}(\Omega)^{*}\right) \quad \text { for all } \epsilon>0,
$$

and:

(1) $\xi$ in (2.45) is given by

$$
\xi(x, t)=-\mathcal{J}_{\{\chi=0\}}(x, t)\left(\gamma(\chi(x, t))+b^{\prime}(\chi(x, t)) \frac{\varepsilon(\mathbf{u}(x, t)) \mathbb{E}(x) \varepsilon(\mathbf{u}(x, t))}{2}-\vartheta(x, t)\right)^{+},
$$

for almost all $(x, t) \in \Omega \times(0, T)$, where $\mathcal{J}_{\{\chi=0\}}$ denotes the characteristic function of the set $\{(x, t) \in$ $\Omega \times(0, T): \chi(x, t)=0\}$,

(2) $\exists \underline{\vartheta}>0$ such that

$$
\vartheta(x, t) \geq \underline{\vartheta}>0 \quad \text { for a.a. }(x, t) \in \Omega \times(0, T) .
$$

Furthermore, if in addition $\mathrm{K}$ satisfies Hypothesis (V), there holds

$$
\vartheta \in \mathrm{BV}\left([0, T] ; W^{2, d+\epsilon}(\Omega)^{*}\right) \quad \text { for every } \epsilon>0,
$$

and the total energy inequality (2.40) holds for all $t \in[0, T]$, for $s=0$, and for almost all $s \in(0, t)$.

Observe that (2.51) yields that there exists $D \subset[0, T]$, at most infinitely countable, such that $\vartheta \in \mathrm{C}^{0}([0, T] \backslash$ $\left.D ; W^{2, d+\epsilon}(\Omega)^{*}\right)$. We will develop the proof in Section 5 by passing to the limit in the time-discretization scheme carefully devised in Section 4

Remark 2.9 (Uniqueness and extensions). (1) Uniqueness of solutions for the irreversible system, even in the isothermal case, is still an open problem. This is mainly due to the doubly nonlinear character of (1.3) (cf. also [11] for non-uniqueness examples for a general doubly nonlinear equation).

(2) Theorem 1 could be easily extended to the case in which the indicator function $I_{(-\infty, 0]}$ in (1.3) is replaced by

$\widehat{\alpha}: \mathbb{R} \rightarrow[0,+\infty]$ convex, 1-positively homogeneous, with $\operatorname{dom}(\widehat{\alpha}) \subset(-\infty, 0]$ and $0 \in \alpha(0)$.

2.4. A global existence result for the reversible system. In the case $\mu=0$, we are able to cope with a weak solvability notion for system (1.1)-(1.3) stronger than the one from Definition 2.5. Indeed, it features a pointwise formulation for the internal parameter equation (1.3), while keeping the entropic formulation for the heat equation (1.1). Under the additional Hypothesis (V), we will also improve the weak formulation of the heat equation (cf. (2.57) below). As a byproduct, we will manage to prove the total energy identity for all $t \in[0, T]$.

Definition 2.10 (Entropic solutions to the reversible system). Let $\mu=0$. Given initial data $\left(\vartheta_{0}, \mathbf{u}_{0}, \mathbf{v}_{0}, \chi_{0}\right)$ fulfilling (2.26)-(2.28), we call a triple $(\vartheta, \mathbf{u}, \chi)$ an entropic solution to the (initial-boundary value problem) for system (1.1)-(1.3), with the boundary conditions (1.4), if it has the regularity (2.33)-(2.36), it complies with the initial conditions (2.37)-(2.38), and with

- the entropy inequality (2.39); 
- the total energy inequality (2.40) for almost all $t \in(0, T]$, for $s=0$, and for almost all $s \in(0, t)$;

- the momentum equation (2.42);

- the internal parameter equation

$$
\chi_{t}+A_{p} \chi+\xi+\gamma(\chi)=-b^{\prime}(\chi) \frac{\varepsilon(\mathbf{u}) \mathbb{E} \varepsilon(\mathbf{u})}{2}+\vartheta \quad \text { a.e. in } \Omega \times(0, T),
$$

with

$$
\xi \in L^{2}\left(0, T ; L^{2}(\Omega)\right) \text { s.t. } \quad \xi(x, t) \in \beta(\chi(x, t)) \text { for a.a. }(x, t) \in \Omega \times(0, T) .
$$

Our second main result states the existence of an entropic solution $(\vartheta, \mathbf{u}, \chi)$ (in the sense of Definition 2.10) to the PDE system (1.1)-(1.3). Furthermore, we show that, under the additional Hypothesis (V), the formulation of the heat equation (1.1) improves to a standard variational formulation (cf. (2.57) below), albeit with suitably smooth test functions, and the total energy inequality (2.40) holds as an equality. We shall refer to the solutions thus obtained as weak.

Theorem 2 (Existence of entropic and weak solutions, $\mu=0$ ). Let $\mu=0$. Assume Hypotheses (0)-(III), and conditions (2.23)-(2.28) on the data $\mathbf{f}, g, h, \vartheta_{0}, \mathbf{u}_{0}, \mathbf{v}_{0}, \chi_{0}$. Then, there exists an entropic solution (in the sense of Definition 2.10) $(\vartheta, \mathbf{u}, \chi)$ to the initial-boundary value problem for system (1.1)-(1.3), such that the strict positivity property (2.50) holds for $\vartheta$, and such that $\chi$ has the enhanced regularity

$$
\chi \in L^{2}\left(0, T ; W^{1+\sigma, p}(\Omega)\right) \quad \text { for all } 0<\sigma<\frac{1}{p} .
$$

Moreover, if $\mathrm{K}$ also complies with Hypothesis ( $\mathbf{V})$, then $\vartheta$ has the enhanced regularity

$$
\vartheta \in W^{1,1}\left(0, T ; W^{2, d+\epsilon}(\Omega)^{*}\right) \quad \text { for every } \epsilon>0
$$

(cf. (2.51) $)$, and the heat equation (1.1) is fulfilled in the following improved form for almost all $t \in(0, T)$

$$
\begin{aligned}
& \left\langle\partial_{t} \vartheta, \varphi\right\rangle_{W^{2, d+\epsilon}(\Omega)}+\int_{\Omega} \chi_{t} \vartheta \varphi \mathrm{d} x+\rho \int_{\Omega} \operatorname{div}\left(\mathbf{u}_{t}\right) \vartheta \varphi \mathrm{d} x+\int_{\Omega} \mathrm{K}(\vartheta) \nabla \vartheta \nabla \varphi \mathrm{d} x \\
& \left.=\int_{\Omega}\left(g+\frac{\varepsilon\left(\mathbf{u}_{t}\right) \mathbb{V} \varepsilon\left(\mathbf{u}_{t}\right)}{2}+\left|\chi_{t}\right|^{2}\right) \varphi \mathrm{d} x+\int_{\partial \Omega} h \varphi \mathrm{d} S \quad \text { for all } \varphi \in W^{2, d+\epsilon}(\Omega)\right) \text { for some } \epsilon>0 .
\end{aligned}
$$

In this case, the triple $(\vartheta, \mathbf{u}, \chi)$ complies with the total energy equality

$$
\mathscr{E}\left(\vartheta(t), \mathbf{u}(t), \mathbf{u}_{t}(t), \chi(t)\right)=\mathscr{E}\left(\vartheta(s), \mathbf{u}(s), \mathbf{u}_{t}(s), \chi(s)\right)+\int_{s}^{t} \int_{\Omega} g \mathrm{~d} x \mathrm{~d} r+\int_{0}^{t} \int_{\partial \Omega} h \mathrm{~d} S \mathrm{~d} r+\int_{s}^{t} \int_{\Omega} \mathbf{f} \cdot \mathbf{u}_{t} \mathrm{~d} x \mathrm{~d} r,
$$

for all $0 \leq s \leq t \leq T$.

The proof will be given in Section 5 , passing to the limit in the time-discretization scheme set up in Sec. 4. We mention in advance that the argument for (2.57) and for the total energy identity (2.58) for all $t \in[0, T]$ relies on obtaining, for the sequence $\left(\mathbf{u}_{k}, \chi_{k}\right)$ of approximate solutions, the strong convergences

$$
\mathbf{u}_{k} \rightarrow \mathbf{u} \quad \text { in } H^{1}\left(0, T ; H_{0}^{1}\left(\Omega ; \mathbb{R}^{d}\right)\right), \quad \chi_{k} \rightarrow \chi \quad \text { in } H^{1}\left(0, T ; L^{2}(\Omega)\right) .
$$

This allows us to pass to the limit on the right-hand side of the approximate version of (2.57). In turn, the proof of (2.59) is based on a limsup-argument, for which it is essential to have preliminarily obtained the pointwise formulation (2.53) of the equation for $\chi$. This is the reason why we have not been able to obtain the improved formulation (2.57) in the irreversible case $\mu=1$.

Remark 2.11 (Uniqueness in the reversible case). As in the irreversible case, a uniqueness result for the full system seems to be out of reach. Instead, for the isothermal case in [47, Thm. 3] we have proved uniqueness and continuous dependence of the solutions on the data. This result has been obtained in the case that the $p$-Laplacian operator $-\operatorname{div}\left(|\nabla \chi|^{p-2} \nabla \chi\right)$ is replaced by an elliptic operator of the type described in Remark 2.4 , fulfilling an additional non-degeneracy condition, cf. Hypothesis (VII) in [47]: for instance, we may consider $-\operatorname{div}\left(\left(1+|\nabla \chi|^{2}\right)^{(p-2) / 2} \nabla \chi\right)$. 
Remark 2.12 (Alternative boundary conditions for the displacement). Our existence results Theorems 1 and 2 carry over to the case of a time-dependent Dirichlet loading $g$ (in place of the homogeneous Dirichlet condition in (1.4) ) for the displacement $\mathbf{u}$, under suitable conditions on $g$. The latter have to ensure the validity of the elliptic regularity estimate on $\mathbf{u}$ (cf. the forthcoming Fifth estimate in Sec. 3), which leads to the regularity (2.35) and plays a crucial role for our analysis.

Moreover, the proofs of Thms. 1 and 2 could be carried out with suitable modifications in the case of Neumann boundary conditions for $\mathbf{u}$ on the whole of $\partial \Omega$, as well. We would also be able to handle the case of Neumann conditions on a portion $\Gamma_{0}$ of $\partial \Omega$ and Dirichlet conditions on $\Gamma_{1}:=\partial \Omega \backslash \Gamma_{0}\left(\left|\Gamma_{0}\right|,\left|\Gamma_{1}\right|>0\right)$, provided that the closures of the sets $\Gamma_{0}$ and $\Gamma_{1}$ do not intersect. Indeed, without the latter geometric condition, the elliptic regularity results at the core of the Fifth estimate and thus of (2.35) may fail to hold, see [10, Chap. VI, Sec. 6.3].

Nonetheless, in Sec.6, where we will address the analysis of system (1.1)-(1.3), with unidirectional evolution $(\mu=1)$, in the case the $p$-Laplacian regularization in (1.3) is replaced by the Laplace operator, more general boundary conditions on $\mathbf{u}$ could be considered. Indeed, therein we will not be in the position to perform any elliptic regularity estimate on $\mathbf{u}$ (and therefore we will conclude the existence of a weaker notion of solution). Hence, mixed Dirichlet-Neumann conditions on $\mathbf{u}$ could be taken into account in that setting (cf. also Remark 6.3).

\section{3. (Formal) A priori estimates}

In this section, we perform a series of formal estimates on system (1.1)-(1.3). All of these estimates will be rigorously justified on the time-discrete approximation scheme proposed in Section 4, with the exception of the Sixth estimate, to be rendered in a weaker version, cf. the comments prior to the statement of Proposition 4.10, and Remark 4.11,

Yet, we believe that, in order to enhance the readability of the paper, it is worthwhile to develop all the significant calculations on the (easier) time-continuous level. This is especially useful for the Second and the Third a priori estimates, which have a non-standard character and are in fact tailored to handle the quadratic terms on the right-hand side of (1.1).

More in detail, we start by showing the strict positivity of the temperature $\vartheta$, via a comparison argument in the same lines as the one for proving positivity in [16, Subsection 4.2.1]. All the ensuing estimates rely on this property, starting from the basic energy estimate (i.e. the one corresponding to the total energy inequality (2.40)). After this, we test (1.1) by $\vartheta^{\alpha-1}$, with $\alpha \in(0,1)$. This enables us somehow to confine the troublesome quadratic terms to the left-hand side. Carefully using the Gagliardo-Nirenberg inequality, we infer a bound for $\vartheta^{\alpha}$ in $L^{2}\left(0, T ; H^{1}(\Omega)\right)$. Ultimately, exploiting the fact that the heat flux $\mathrm{K}$ controls $\vartheta^{\kappa}$ (cf. (2.17)) we conclude an estimate for $\vartheta$ in $L^{2}\left(0, T ; H^{1}(\Omega)\right)$. This being done, we are in the position to perform all the remaining estimates, i.e. subtracting the temperature equation tested by 1 from the total energy inequality (2.40); performing an elliptic regularity estimate on the momentum equation (1.3), and comparison estimates in (1.1) and (1.3).

We mention in advance that, with the exception of the last one, all of the ensuing estimates hold both in the reversible $(\mu=0)$, and in the irreversible $(\mu=1)$ cases. We warn the reader that in what follows we will use the same symbol $C$ for several different constants, even varying from line to line and depending only on the data of the problem, on $\Omega$ and on $T$.

Positivity of $\vartheta[\mu \in\{0,1\}]$. Moving all the quadratic terms in (1.1) to the right-hand side, we obtain

$$
\begin{aligned}
\vartheta_{t}-\operatorname{div}(\mathrm{K}(\vartheta) \nabla \vartheta) & =g+a(\chi) \varepsilon\left(\mathbf{u}_{t}\right) \mathbb{V} \varepsilon\left(\mathbf{u}_{t}\right)+\left|\chi_{t}\right|^{2}-\chi_{t} \vartheta-\rho \vartheta \operatorname{div}\left(\mathbf{u}_{t}\right) \\
& \geq g+c\left|\varepsilon\left(\mathbf{u}_{t}\right)\right|^{2}+\frac{1}{2}\left|\chi_{t}\right|^{2}-C \vartheta^{2} \geq-C \vartheta^{2} \quad \text { a.e. in } \Omega \times(0, T),
\end{aligned}
$$


where we have written (1.1) in a formal way, disregarding the (positive) boundary datum $h$. Indeed, for the first inequality we have used that $\mathbb{V}$ is positive definite, that $a$ is strictly positive, and the fact that

$$
\left|\operatorname{div}\left(\mathbf{u}_{t}\right)\right| \leq c(d)\left|\varepsilon\left(\mathbf{u}_{t}\right)\right| \quad \text { a.e. in } \Omega \times(0, T)
$$

with $c(d)$ a positive constant only depending on the space dimension $d$. The second estimate also relies on the fact that $g \geq 0$ a.e. in $\Omega \times(0, T)$. Therefore we conclude that $v$ solving the Cauchy problem

$$
v_{t}=-\frac{1}{2} v^{2}, \quad v(0)=\vartheta_{*}>0
$$

is a subsolution of (1.1). Hence, a comparison argument yields

$$
\vartheta(\cdot, t) \geq v(t)>\vartheta_{*}>0 \quad \text { for all } t \in[0, T] .
$$

First estimate $[\mu \in\{0,1\}]$. Test (1.1) by 1 , (1.2) by $\mathbf{u}_{t}$, (1.3) by $\chi_{t}$ and integrate over $(0, t), t \in(0, T]$. Adding the resulting equations and taking into account cancellations, we obtain

$$
\begin{aligned}
& \int_{\Omega} \vartheta(t) \mathrm{d} x+\frac{1}{2} \int_{\Omega}\left|\mathbf{u}_{t}(t)\right|^{2} \mathrm{~d} x+\frac{1}{2} \mathrm{e}(b(\chi(t)) \mathbf{u}(t), \mathbf{u}(t))+\frac{1}{p} \int_{\Omega}|\nabla \chi(t)|^{p} \mathrm{~d} x+\int_{\Omega} W(\chi(t)) \mathrm{d} x \\
& =\int_{\Omega} \vartheta_{0} \mathrm{~d} x+\frac{1}{2} \int_{\Omega}\left|\mathbf{v}_{0}\right|^{2} \mathrm{~d} x+\frac{1}{2} \mathrm{e}\left(b\left(\chi_{0}\right) \mathbf{u}_{0}, \mathbf{u}_{0}\right)+\frac{1}{p} \int_{\Omega}\left|\nabla \chi_{0}\right|^{p} \mathrm{~d} x+\int_{\Omega} W\left(\chi_{0}\right) \mathrm{d} x \\
& +\int_{0}^{t} \int_{\Omega} g \mathrm{~d} x \mathrm{~d} s+\int_{0}^{t} \int_{\partial \Omega} h \mathrm{~d} S \mathrm{~d} s+\int_{0}^{t} \int_{\Omega} \mathbf{f} \cdot \mathbf{u}_{t} \mathrm{~d} x \mathrm{~d} s,
\end{aligned}
$$

viz. the total energy equality (2.58). For (3.3), we have also used the integration-by-parts formula

$$
\int_{0}^{t} \mathrm{e}\left(b(\chi(t)) \mathbf{u}(s), \mathbf{u}_{t}(s)\right) \mathrm{d} s+\frac{1}{2} \int_{0}^{t} \int_{\Omega} b^{\prime}(\chi) \chi_{t} \varepsilon(\mathbf{u}) \mathbb{E} \varepsilon(\mathbf{u}) \mathrm{d} x \mathrm{~d} s=\frac{1}{2} \mathrm{e}(b(\chi(t)) \mathbf{u}(t), \mathbf{u}(t))-\frac{1}{2} \mathrm{e}\left(b\left(\chi_{0}\right) \mathbf{u}_{0}, \mathbf{u}_{0}\right)
$$

as well as the fact that $\int_{0}^{t} \int_{\Omega} \partial I_{(-\infty, 0]}\left(\chi_{t}\right) \chi_{t} \mathrm{~d} x \mathrm{~d} s=\int_{0}^{t} \int_{\Omega} I_{(-\infty, 0]}\left(\chi_{t}\right) \mathrm{d} x \mathrm{~d} s=0$ (where we have formally written $\partial I_{(-\infty, 0]}\left(\chi_{t}\right)$ as a single-valued operator). Using (2.23) (2.28) for the data $f, g, h$ and the initial data $\left(\vartheta_{0}, \mathbf{u}_{0}, \chi_{0}\right)$, the positivity of $\vartheta$ (cf. (3.2)), and the fact that $W$ is bounded from below (cf. (2.20) from Hypothesis (III)), also in view of the Poincaré inequality we conclude the following estimate

$$
\|\vartheta\|_{L^{\infty}\left(0, T ; L^{1}(\Omega)\right)}+\|\mathbf{u}\|_{W^{1, \infty}\left(0, T ; L^{2}\left(\Omega ; \mathbb{R}^{d}\right)\right)}+\left\|b(\chi)^{1 / 2} \varepsilon(\mathbf{u})\right\|_{L^{\infty}\left(0, T ; L^{2}\left(\Omega ; \mathbb{R}^{d \times d}\right)\right)}+\|\nabla \chi\|_{L^{p}\left(0, T ; L^{p}(\Omega)\right)} \leq C,
$$

as well as

$$
\|W(\chi)\|_{L^{\infty}\left(0, T ; L^{1}(\Omega)\right)} \leq C .
$$

Second estimate $[\mu \in\{0,1\}]$. Let $F(\vartheta)=\vartheta^{\alpha} / \alpha$, with $\alpha \in(0,1)$. We test (1.1) by $F^{\prime}(\vartheta):=\vartheta^{\alpha-1}$, and integrate on $(0, t)$ with $t \in(0, T]$. We thus have

$$
\begin{aligned}
& \int_{\Omega} F\left(\vartheta_{0}\right) \mathrm{d} x+\int_{0}^{t} \int_{\Omega} g F^{\prime}(\vartheta) \mathrm{d} x \mathrm{~d} s+\int_{0}^{t} \int_{\partial \Omega} h F^{\prime}(\vartheta) \mathrm{d} S \mathrm{~d} s+\int_{0}^{t} \int_{\Omega} a(\chi) \varepsilon\left(\mathbf{u}_{t}\right) \mathbb{V} \varepsilon\left(\mathbf{u}_{t}\right) F^{\prime}(\vartheta) \mathrm{d} x \mathrm{~d} s \\
& +\int_{0}^{t} \int_{\Omega}\left|\chi_{t}\right|^{2} F^{\prime}(\vartheta) \mathrm{d} x \mathrm{~d} s=\int_{\Omega} F(\vartheta(t)) \mathrm{d} x+\int_{0}^{t} \int_{\Omega} \chi_{t} \vartheta F^{\prime}(\vartheta) \mathrm{d} x \mathrm{~d} s+\rho \int_{0}^{t} \int_{\Omega} \vartheta \operatorname{div}\left(\mathbf{u}_{t}\right) F^{\prime}(\vartheta) \mathrm{d} x \mathrm{~d} s \\
& +\int_{0}^{t} \int_{\Omega} \mathrm{K}(\vartheta) \nabla \vartheta \nabla\left(F^{\prime}(\vartheta)\right) \mathrm{d} x \mathrm{~d} s
\end{aligned}
$$

whence (cf. (2.6), (2.4), and the positivity (2.24) and (2.25) of $g$ and $h$ )

$$
\begin{aligned}
& \frac{4(1-\alpha)}{\alpha^{2}} \int_{0}^{t} \int_{\Omega} \mathrm{K}(\vartheta)\left|\nabla\left(\vartheta^{\alpha / 2}\right)\right|^{2} \mathrm{~d} x \mathrm{~d} s+c_{2} \beta_{0} \int_{0}^{t} \int_{\Omega}\left|\varepsilon\left(\mathbf{u}_{t}\right)\right|^{2} F^{\prime}(\vartheta) \mathrm{d} x \mathrm{~d} s+\int_{0}^{t} \int_{\Omega}\left|\chi_{t}\right|^{2} F^{\prime}(\vartheta) \mathrm{d} x \mathrm{~d} s \\
& \leq \int_{\Omega}\left|F\left(\vartheta_{0}\right)\right| \mathrm{d} x+I_{1}+I_{2}+I_{3},
\end{aligned}
$$


where we have used (2.6) (with $\beta_{0}$ from (2.4)), and (2.18). We estimate

$$
I_{1}=\int_{\Omega}|F(\vartheta(t))| \mathrm{d} x \leq \frac{1}{\alpha} \int_{\Omega} \max \{\vartheta(t), 1\}^{\alpha} \mathrm{d} x \leq \frac{1}{\alpha} \int_{\Omega} \max \{\vartheta(t), 1\} \mathrm{d} x \leq C
$$

since $\alpha<1$ and taking into account the previously obtained (3.5). Analogously we can estimate $\int_{\Omega}\left|F\left(\vartheta_{0}\right)\right| \mathrm{d} x$; moreover,

$$
I_{2}=\int_{0}^{t} \int_{\Omega}\left|\chi_{t} \vartheta F^{\prime}(\vartheta)\right| \mathrm{d} x \mathrm{~d} s \leq \frac{1}{4} \int_{0}^{t} \int_{\Omega}\left|\chi_{t}\right|^{2} F^{\prime}(\vartheta) \mathrm{d} x \mathrm{~d} s+\int_{0}^{t} \int_{\Omega} F^{\prime}(\vartheta) \vartheta^{2} \mathrm{~d} x \mathrm{~d} s .
$$

Using (2.18), inequality (3.1), and Young's inequality, we have that

$$
I_{3}=|\rho| \int_{0}^{t} \int_{\Omega}\left|\vartheta \operatorname{div}\left(\mathbf{u}_{t}\right) F^{\prime}(\vartheta)\right| \mathrm{d} x \mathrm{~d} s \leq \frac{\beta_{0} c_{2}}{4} \int_{0}^{t} \int_{\Omega}\left|\varepsilon\left(\mathbf{u}_{t}\right)\right|^{2} F^{\prime}(\vartheta) \mathrm{d} x \mathrm{~d} s+C \int_{0}^{t} \int_{\Omega} F^{\prime}(\vartheta) \vartheta^{2} \mathrm{~d} x \mathrm{~d} s .
$$

All in all, we conclude

$$
\begin{aligned}
& \frac{4(1-\alpha)}{\alpha^{2}} \int_{0}^{t} \int_{\Omega} \mathrm{K}(\vartheta)\left|\nabla\left(\vartheta^{\alpha / 2}\right)\right|^{2} \mathrm{~d} x \mathrm{~d} s+\frac{3 \beta_{0} c_{2}}{4} \int_{0}^{t} \int_{\Omega}\left|\varepsilon\left(\mathbf{u}_{t}\right)\right|^{2} F^{\prime}(\vartheta) \mathrm{d} x \mathrm{~d} s+\frac{3}{4} \int_{0}^{t} \int_{\Omega}\left|\chi_{t}\right|^{2} F^{\prime}(\vartheta) \mathrm{d} x \mathrm{~d} s \\
& \leq C+C \int_{0}^{t} \int_{\Omega} \vartheta^{\alpha+1} \mathrm{~d} x \mathrm{~d} s .
\end{aligned}
$$

Now, we fix $q \geq 4$ and introduce the auxiliary quantity $\eta:=\max \{\vartheta, 1\}$. Observe that $\eta$ is still in $H^{1}(\Omega)$, and that, for $q$ sufficiently big (see below) we have

$$
\frac{\alpha}{2} \geq \frac{\alpha+1}{q} \text { whence } \eta^{(\alpha+1) / q} \leq \eta^{\alpha / 2} \doteq w .
$$

Therefore, taking into account that

$$
\int_{0}^{t} \int_{\Omega} \mathrm{K}(\vartheta)\left|\nabla\left(\vartheta^{\alpha / 2}\right)\right|^{2} \mathrm{~d} x \mathrm{~d} s \geq c_{1} \iint_{\{\vartheta \geq 1\}}\left|\nabla\left(\vartheta^{\alpha / 2}\right)\right|^{2} \mathrm{~d} x \mathrm{~d} s=c_{1} \int_{0}^{t} \int_{\Omega}|\nabla w|^{2} \mathrm{~d} x \mathrm{~d} s,
$$

thanks to (2.17), we infer from (3.7) and (3.8) that

$$
\int_{0}^{t} \int_{\Omega}|\nabla w|^{2} \mathrm{~d} x \mathrm{~d} s \leq C+C \int_{0}^{t}\|w\|_{L^{q}(\Omega)}^{q} \mathrm{~d} s .
$$

We now apply the Gagliardo-Nirenberg inequality for $d=3$ (for $d=2$ even better estimates hold true), yielding

$$
\|w\|_{L^{q}(\Omega)} \leq c_{1}\|\nabla w\|_{L^{2}\left(\Omega ; \mathbb{R}^{d}\right)}^{\theta}\|w\|_{L^{r}(\Omega)}^{1-\theta}+c_{2}\|w\|_{L^{r}(\Omega)}
$$

with $1 \leq r \leq q$ and $\theta$ satisfying $1 / q=\theta / 6+(1-\theta) / r$. Hence $\theta=6(q-r) / q(6-r)$. Observe that $\theta \in(0,1)$ if $q<6$ and that this restriction on $q$ implies that, for (3.8) we need to have $\alpha \in(1 / 2,1)$. Plugging the Gagliardo-Nirenberg estimate into (3.9), using Young's inequality with exponents $2 / \theta q$ and $2 /(2-\theta q)$ and with suitable weights in such a way as to absorb the term $\|\nabla w\|_{L^{2}\left(\Omega ; \mathbb{R}^{d}\right)}^{2}$ into the left-hand side of (3.9)), we ultimately conclude

$$
\frac{c}{2} \int_{0}^{t} \int_{\Omega}|\nabla w|^{2} \mathrm{~d} x \mathrm{~d} s \leq C+C \int_{0}^{t}\|w\|_{L^{r}(\Omega)}^{2 q(1-\theta) /(2-q \theta)} \mathrm{d} s+C^{\prime} \int_{0}^{t}\|w\|_{L^{r}(\Omega)}^{q} \mathrm{~d} s .
$$

Now, choosing $r \leq 2 / \alpha$, we have that

$$
\|w\|_{L^{r}(\Omega)}=\left(\int_{\Omega} \eta^{r \alpha / 2} \mathrm{~d} x\right)^{1 / r} \leq\left(\int_{\Omega} \eta \mathrm{d} x\right)^{1 / r} \leq C\|\vartheta\|_{L^{\infty}\left(0, T ; L^{1}(\Omega)\right)}+|\Omega| \leq C,
$$

where the latter inequality is due to estimate (3.5). Combining the above estimate with (3.11) we infer a bound for $w=\eta^{\alpha / 2}$ in $L^{2}\left(0, T ; H^{1}(\Omega)\right) \cap L^{\infty}\left(0, T ; L^{r}(\Omega)\right)$. Ultimately, also in view of (3.9), we conclude that

$$
\left\|\vartheta^{\alpha / 2}\right\|_{L^{2}\left(0, T ; H^{1}(\Omega)\right) \cap L^{\infty}\left(0, T ; L^{r}(\Omega)\right)} \leq C .
$$


Third estimate $[\mu \in\{0,1\}]$. It follows from (3.7) and (2.17) that

$$
\begin{aligned}
C \geq \int_{0}^{t} \int_{\Omega} \mathrm{K}(\vartheta)\left|\nabla\left(\vartheta^{\alpha / 2}\right)\right|^{2} \mathrm{~d} x \mathrm{~d} s & \geq c_{1} \int_{0}^{t} \int_{\Omega} \vartheta^{\kappa}\left|\nabla\left(\vartheta^{\alpha / 2}\right)\right|^{2} \mathrm{~d} x \mathrm{~d} s \\
& =\int_{0}^{t} \int_{\Omega}\left|\vartheta^{\kappa+\alpha-2}\right||\nabla \vartheta|^{2} \mathrm{~d} x \mathrm{~d} s \\
& =\int_{0}^{t} \int_{\Omega}\left|\nabla\left(\vartheta^{(\kappa+\alpha) / 2}\right)\right|^{2} \mathrm{~d} x \mathrm{~d} s
\end{aligned}
$$

with $\alpha \in[1 / 2,1)$ arbitrary.

From (3.13) and the strict positivity of $\vartheta(3.2)$ it follows that

$$
\int_{0}^{t} \int_{\Omega}|\nabla \vartheta|^{2} \mathrm{~d} x \mathrm{~d} s \leq C
$$

provided that $\kappa+\alpha-2 \geq 0$. Observe that, since $\kappa>1$ we can choose $\alpha \in[1 / 2,1)$ such that this inequality holds. Hence, taking into account estimate (3.5) and applying Poincaré inequality, we deduce

$$
\|\vartheta\|_{L^{2}\left(0, T ; H^{1}(\Omega)\right)} \leq C .
$$

We now interpolate between estimate (3.14) and estimate (3.5) for $\|\vartheta\|_{L^{\infty}\left(0, T ; L^{1}(\Omega)\right)}$, using the GagliardoNirenberg inequality (2.11) that gives $\|\vartheta\|_{L^{h}(\Omega)} \leq\|\vartheta\|_{H^{1}(\Omega)}^{\theta}\|\vartheta\|_{L^{1}(\Omega)}^{1-\theta}$ with $\theta \in(0,1)$ and $h \in[1, \infty]$ related by $\frac{1}{h}=\theta\left(\frac{1}{2}-\frac{1}{d}\right)+1-\theta$. Hence, we get

$$
\|\vartheta\|_{L^{h}(\Omega \times(0, T))} \leq C \quad \text { with } h=8 / 3 \quad \text { if } d=3, \quad h=3 \quad \text { if } d=2 .
$$

For later use, we also point out that

$$
\left\|\nabla \vartheta^{(\kappa-\alpha) / 2}\right\|_{L^{2}\left(0, T ; L^{2}(\Omega)\right)} \leq C
$$

Indeed, it suffices to observe that

$$
\int_{\Omega}\left|\nabla \vartheta^{(\kappa-\alpha) / 2}\right|^{2} \mathrm{~d} x=\int_{\Omega} \vartheta^{\kappa-\alpha-2}|\nabla \vartheta|^{2} \mathrm{~d} x \leq \frac{1}{\vartheta_{*}^{2 \alpha}} \int_{\Omega} \vartheta^{\kappa+\alpha-2}|\nabla \vartheta|^{2} \mathrm{~d} x \leq C,
$$

where the first inequality derives from the positivity property (3.2), and the last one from estimate (3.13). Combining (3.13) and (3.16) with estimate (3.5), and using a nonlinear version of the Poincaré inequality (cf. e.g. (2.14)), we infer

$$
\left\|\vartheta^{(\kappa-\alpha) / 2}\right\|_{L^{2}\left(0, T ; H^{1}(\Omega)\right)},\left\|\vartheta^{(\kappa+\alpha) / 2}\right\|_{L^{2}\left(0, T ; H^{1}(\Omega)\right)} \leq C .
$$

Fourth estimate $[\mu \in\{0,1\}]$. We test (1.1) by 1 , integrate over $(0, t)$, and subtract the resulting identity from the total energy balance (3.3). We thus obtain

$$
\begin{aligned}
& \frac{1}{2} \int_{\Omega}\left|\mathbf{u}_{t}(t)\right|^{2} \mathrm{~d} x+\int_{0}^{t} \mathbf{v}\left(a(\chi) \mathbf{u}_{t}, \mathbf{u}_{t}\right) \mathrm{d} s+\frac{1}{2} \mathrm{e}(b(\chi(t)) \mathbf{u}(t), \mathbf{u}(t))+\int_{0}^{t} \int_{\Omega}\left|\chi_{t}\right|^{2} \mathrm{~d} x \mathrm{~d} s+\int_{\Omega} \frac{1}{p}|\nabla \chi(t)|^{p}+W(\chi(t)) \mathrm{d} x \\
& =\frac{1}{2} \int_{\Omega}\left|\mathbf{u}_{0}\right|^{2} \mathrm{~d} x+\frac{1}{2} \mathrm{e}\left(b\left(\chi_{0}\right) \mathbf{u}_{0}, \mathbf{u}_{0}\right)+\int_{\Omega} \frac{1}{p}\left|\nabla \chi_{0}\right|^{p}+\int_{\Omega} W\left(\chi_{0}\right) \mathrm{d} x+\int_{0}^{t} \int_{\Omega} \vartheta\left(\rho \operatorname{div} \mathbf{u}_{t}+\chi_{t}\right) \mathrm{d} x \mathrm{~d} s \\
& \quad+\int_{0}^{t} \int_{\Omega} \mathbf{f} \mathbf{u}_{t} \mathrm{~d} x \mathrm{~d} s .
\end{aligned}
$$

Using now (2.27)-(2.28) to estimate the initial data $\left(\mathbf{u}_{0}, \chi_{0}\right)$, (2.23) on $\mathbf{f}$, Hyp. (III) (which in particular yields that $W$ is bounded from below), and combining estimate (3.14) on $\vartheta$ with (3.1), we obtain

$$
\left\|\chi_{t}\right\|_{L^{2}(\Omega \times(0, T))}+\left\|a(\chi)^{1 / 2} \varepsilon\left(\mathbf{u}_{t}\right)\right\|_{L^{2}\left(\Omega \times(0, T) ; \mathbb{R}^{d \times d}\right)} \leq C,
$$

whence $\left\|\mathbf{u}_{t}\right\|_{L^{2}\left(0, T ; H_{0}^{1}\left(\Omega ; \mathbb{R}^{d}\right)\right)} \leq C$, by (2.18). Furthermore, in view of (3.5) we also gather

$$
\|\chi\|_{L^{\infty}\left(0, T ; W^{1, p}(\Omega)\right)} \leq C .
$$


Fifth estimate $[\mu \in\{0,1\}]$. We use here the crucial assumption that $p>d$. We test (1.2) by $-\operatorname{div}\left(\mathbb{V} \varepsilon\left(\mathbf{u}_{t}\right)\right)$ and integrate on time (cf. also [47, Sec. 3]), thus obtaining

$$
\begin{aligned}
& -\int_{0}^{t} \mathbf{u}_{t t} \cdot \operatorname{div}\left(\mathbb{V} \varepsilon\left(\mathbf{u}_{t}\right)\right) \mathrm{d} x \mathrm{~d} s+\int_{0}^{t} \int_{\Omega} \operatorname{div}\left(a(\chi) \mathbb{V} \varepsilon\left(\mathbf{u}_{t}\right)\right) \cdot \operatorname{div}\left(\mathbb{V} \varepsilon\left(\mathbf{u}_{t}\right)\right) \mathrm{d} x \mathrm{~d} s \\
& =-\int_{0}^{t} \int_{\Omega} \operatorname{div}(b(\chi) \mathbb{E} \varepsilon(\mathbf{u})) \cdot \operatorname{div}\left(\mathbb{V} \varepsilon\left(\mathbf{u}_{t}\right)\right) \mathrm{d} x \mathrm{~d} s+\rho \int_{0}^{t} \int_{\Omega} \nabla \vartheta \cdot \operatorname{div}\left(\mathbb{V} \varepsilon\left(\mathbf{u}_{t}\right)\right) \mathrm{d} x \mathrm{~d} s-\int_{0}^{t} \int_{\Omega} \mathbf{f} \cdot \operatorname{div}\left(\mathbb{V} \varepsilon\left(\mathbf{u}_{t}\right)\right) \mathrm{d} x \mathrm{~d} s .
\end{aligned}
$$

Then, we consider the occurring terms individually. The kinetic term gives

$$
\int_{0}^{t} \int_{\Omega} \mathbf{u}_{t t} \operatorname{div}\left(\mathbb{V} \varepsilon\left(\mathbf{u}_{t}\right)\right) \mathrm{d} x \mathrm{~d} s=\int_{\Omega} \frac{1}{2} \varepsilon\left(\mathbf{u}_{t}(t)\right) \mathbb{V} \varepsilon\left(\mathbf{u}_{t}(t)\right) \mathrm{d} x-\int_{\Omega} \frac{1}{2} \varepsilon\left(\mathbf{u}_{t}(0)\right) \mathbb{V} \varepsilon\left(\mathbf{u}_{t}(0)\right) \mathrm{d} x .
$$

For the viscous term on the left-hand side of (3.21) we rely on (2.18) and the elliptic regularity result in (2.10) and we obtain

$$
\begin{aligned}
\int_{0}^{t} \int_{\Omega} \operatorname{div}\left(a(\chi) \mathbb{V} \varepsilon\left(\mathbf{u}_{t}\right)\right) \cdot \operatorname{div}\left(\mathbb{V} \varepsilon\left(\mathbf{u}_{t}\right)\right) \mathrm{d} x \mathrm{~d} s= & \int_{0}^{t} \int_{\Omega} a(\chi) \operatorname{div}\left(\mathbb{V} \varepsilon\left(\mathbf{u}_{t}\right)\right) \cdot \operatorname{div}\left(\mathbb{V} \varepsilon\left(\mathbf{u}_{t}\right)\right) \mathrm{d} x \mathrm{~d} s \\
& +\int_{0}^{t} \int_{\Omega} \nabla a(\chi) \mathbb{V} \varepsilon\left(\mathbf{u}_{t}\right) \cdot \operatorname{div}\left(\mathbb{V} \varepsilon\left(\mathbf{u}_{t}\right)\right) \mathrm{d} x \mathrm{~d} s \\
& \geq C \int_{0}^{t} \int_{\Omega}\left|\operatorname{div}\left(\mathbb{V} \varepsilon\left(\mathbf{u}_{t}\right)\right)\right|^{2} \mathrm{~d} x \mathrm{~d} s+I_{1} \\
& \geq c \int_{0}^{t}\left\|\mathbf{u}_{t}\right\|_{H^{2}\left(\Omega ; \mathbb{R}^{d}\right)}^{2} \mathrm{~d} s+I_{1} .
\end{aligned}
$$

We then move $I_{1}$ to the right-hand side of (3.21) and estimate

$$
\begin{aligned}
\left|I_{1}\right| & =\left|\int_{0}^{t} \int_{\Omega} \nabla a(\chi) \mathbb{V} \varepsilon\left(\mathbf{u}_{t}\right) \cdot \operatorname{div}\left(\mathbb{V} \varepsilon\left(\mathbf{u}_{t}\right)\right)\right| \mathrm{d} x \mathrm{~d} s \\
& \leq C \int_{0}^{t}\|\nabla a(\chi)\|_{L^{d+\zeta}\left(\Omega ; \mathbb{R}^{d}\right)}\left\|\varepsilon\left(\mathbf{u}_{t}\right)\right\|_{L^{d^{\star}-\zeta}\left(\Omega ; \mathbb{R}^{d \times d}\right)}\left\|\operatorname{div}\left(\mathbb{V} \varepsilon\left(\mathbf{u}_{t}\right)\right)\right\|_{L^{2}\left(\Omega ; \mathbb{R}^{d}\right)} \mathrm{d} s \\
& \leq \delta \int_{0}^{t}\left\|\mathbf{u}_{t}\right\|_{H^{2}\left(\Omega ; \mathbb{R}^{d}\right)}^{2} \mathrm{~d} s+C_{\delta} \int_{0}^{t}\|\nabla a(\chi)\|_{L^{d+\zeta}\left(\Omega ; \mathbb{R}^{d}\right)}^{2}\left\|\varepsilon\left(\mathbf{u}_{t}\right)\right\|_{L^{d^{\star}-\zeta\left(\Omega ; \mathbb{R}^{d \times d}\right)}}^{2} \mathrm{~d} s \\
& \leq \delta \int_{0}^{t}\left\|\mathbf{u}_{t}\right\|_{H^{2}\left(\Omega ; \mathbb{R}^{d}\right)}^{2} \mathrm{~d} s+C_{\delta} \varrho^{2} \int_{0}^{t}\|\chi\|_{W^{1, p}(\Omega)}^{2}\left\|\mathbf{u}_{t}\right\|_{H^{2}\left(\Omega ; \mathbb{R}^{d}\right)}^{2} \mathrm{~d} s+C_{\delta} C_{\varrho} \int_{0}^{t}\|\chi\|_{W^{1, p}(\Omega)}^{2}\left\|\mathbf{u}_{t}\right\|_{L^{2}\left(\Omega ; \mathbb{R}^{d}\right)}^{2} \mathrm{~d} s,
\end{aligned}
$$

exploiting (2.18) and (2.13), for some positive constants $\delta$ and $\varrho$ that we will choose later and for some $C_{\delta}, C_{\varrho}>0$. For the purely elastic contribution on the right-hand side of (3.21) we argue in this way: using the assumption $p>d$, we can fix $\zeta>0$ such that $p \geq d+\zeta$ and we get, due also to (2.18),

$$
\begin{aligned}
& -\int_{0}^{t} \int_{\Omega} \operatorname{div}(b(\chi) \mathbb{E} \varepsilon(\mathbf{u})) \cdot \operatorname{div}\left(\mathbb{V} \varepsilon\left(\mathbf{u}_{t}\right)\right) \mathrm{d} x \mathrm{~d} s \\
& =-\int_{0}^{t} \int_{\Omega} \nabla b(\chi) \mathbb{E} \varepsilon(\mathbf{u}) \operatorname{div}\left(\mathbb{V} \varepsilon\left(\mathbf{u}_{t}\right)\right) \mathrm{d} x \mathrm{~d} s-\int_{0}^{t} \int_{\Omega} b(\chi) \operatorname{div}(\mathbb{E} \varepsilon(\mathbf{u})) \operatorname{div}\left(\mathbb{V} \varepsilon\left(\mathbf{u}_{t}\right)\right) \mathrm{d} x \mathrm{~d} s \\
& \leq C \int_{0}^{t}\|\nabla b(\chi)\|_{L^{d+\zeta}\left(\Omega ; \mathbb{R}^{d}\right)}\|\varepsilon(\mathbf{u})\|_{L^{d^{\star}-\zeta\left(\Omega ; \mathbb{R}^{d \times d}\right)}}\left\|\operatorname{div}\left(\mathbb{V} \varepsilon\left(\mathbf{u}_{t}\right)\right)\right\|_{L^{2}\left(\Omega ; \mathbb{R}^{d}\right)} \mathrm{d} s+C \int_{0}^{t}\|\mathbf{u}\|_{H^{2}\left(\Omega ; \mathbb{R}^{d}\right)}\left\|\mathbf{u}_{t}\right\|_{H^{2}\left(\Omega ; \mathbb{R}^{d}\right)} \mathrm{d} s \\
& \leq \sigma \int_{0}^{t}\left\|\mathbf{u}_{t}\right\|_{H^{2}\left(\Omega ; \mathbb{R}^{d}\right)}^{2} \mathrm{~d} s+C_{\sigma} \int_{0}^{t}\left(\|\chi\|_{W^{1, p}(\Omega)}^{2}\|\mathbf{u}\|_{H^{2}\left(\Omega ; \mathbb{R}^{d}\right)}^{2}+\|\mathbf{u}\|_{H^{2}\left(\Omega ; \mathbb{R}^{d}\right)}^{2}\right) \mathrm{d} s .
\end{aligned}
$$

Here, $d^{\star}$ is from (2.12) and we have exploited inequality (2.13) with a constant $\sigma$ that we will choose later, and some $C_{\sigma}>0$. Moreover, we have used (2.10) and the fact that $\|\nabla(b(\chi))\|_{L^{d+\zeta}(\Omega)} \leq C\|\chi\|_{W^{1, p}(\Omega)}$. For the 
thermal expansion term we have that

$$
\left|\rho \int_{0}^{t} \int_{\Omega} \nabla \vartheta \cdot \operatorname{div}\left(\mathbb{V} \varepsilon\left(\mathbf{u}_{t}\right)\right) \mathrm{d} x \mathrm{~d} s\right| \leq \eta \int_{0}^{t}\left\|\mathbf{u}_{t}\right\|_{H^{2}\left(\Omega ; \mathbb{R}^{d}\right)}^{2} \mathrm{~d} s+C_{\eta} \int_{0}^{t}\|\nabla \vartheta\|_{L^{2}\left(\Omega ; \mathbb{R}^{d}\right)}^{2} \mathrm{~d} s
$$

holds true for some positive constant $\eta$ to be fixed later and for some $C_{\eta}>0$. Collecting (3.22)-(3.25), the previously proved estimates (3.5), (3.14), and exploiting (2.23) on $\mathbf{f}$ to estimate the last term on the right-hand side of (3.21), we conclude that

$$
\begin{aligned}
\frac{\beta_{0}}{2} \int_{\Omega}\left|\varepsilon\left(\mathbf{u}_{t}(t)\right)\right|^{2} \mathrm{~d} x+c \int_{0}^{t}\left\|\mathbf{u}_{t}\right\|_{H^{2}\left(\Omega ; \mathbb{R}^{d}\right)}^{2} \mathrm{~d} s \leq & C \int_{\Omega}\left|\varepsilon\left(\mathbf{v}_{0}\right)\right|^{2} \mathrm{~d} x+C\|\mathbf{f}\|_{L^{2}\left(0, T ; L^{2}\left(\Omega ; \mathbb{R}^{d}\right)\right)}^{2}+\frac{c}{2} \int_{0}^{t}\left\|\mathbf{u}_{t}\right\|_{H^{2}\left(\Omega ; \mathbb{R}^{d}\right)}^{2} \mathrm{~d} s \\
& +C\left(1+\left\|\mathbf{u}_{0}\right\|_{H^{2}\left(\Omega ; \mathbb{R}^{d}\right)}^{2}+\int_{0}^{t} \int_{0}^{s}\left\|\mathbf{u}_{t}\right\|_{H^{2}\left(\Omega ; \mathbb{R}^{d}\right)}^{2} \mathrm{~d} r \mathrm{~d} s\right)
\end{aligned}
$$

with $\beta_{0}$ from (2.4), where we have used the fact that $\int_{0}^{t}\|\mathbf{u}\|_{H^{2}\left(\Omega ; \mathbb{R}^{d}\right)}^{2} \mathrm{~d} s \leq\left\|\mathbf{u}_{0}\right\|_{H^{2}\left(\Omega ; \mathbb{R}^{d}\right)}^{2}+\int_{0}^{t} \int_{0}^{s}\left\|\mathbf{u}_{t}\right\|_{H^{2}\left(\Omega ; \mathbb{R}^{d}\right)}^{2} \mathrm{~d} r \mathrm{~d} s$ and chosen $\sigma, \delta, \varrho$ and $\eta$ sufficiently small. Taking into account condition (2.23) on $\mathbf{f}$, the assumptions on the initial data (2.27), and using a standard Gronwall lemma, we conclude

$$
\left\|\mathbf{u}_{t}\right\|_{L^{2}\left(0, T ; H_{\mathrm{Dir}}^{2}\left(\Omega ; \mathbb{R}^{d}\right)\right) \cap L^{\infty}\left(0, T ; H_{0}^{1}\left(\Omega ; \mathbb{R}^{d}\right)\right)} \leq C .
$$

By comparison in (1.2), taking into account the regularity property (2.9b), we also get

$$
\left\|\mathbf{u}_{t t}\right\|_{L^{2}\left(0, T ; L^{2}\left(\Omega ; \mathbb{R}^{d}\right)\right)} \leq C .
$$

Sixth estimate $[\mu \in\{0,1\}]$. We multiply (1.1) by $\frac{w}{\vartheta}$, with $w$ a test function in $W^{1, d}(\Omega) \cap L^{\infty}(\Omega)$ (in particular, this is true for $w \in W^{1, d+\epsilon}(\Omega)$ with $\left.\epsilon>0\right)$. We integrate in space, only. Thus, using the place-holders $H:=-\chi_{t}-\rho \operatorname{div}\left(\mathbf{u}_{t}\right)$ and $J:=\frac{1}{\vartheta}\left(g+a(\chi) \varepsilon\left(\mathbf{u}_{t}\right) \mathbb{V} \varepsilon\left(\mathbf{u}_{t}\right)+\left|\chi_{t}\right|^{2}\right)$, we obtain (cf. (2.29) ) that

$$
\begin{aligned}
& \left|\int_{\Omega} \partial_{t} \log (\vartheta) w \mathrm{~d} x\right| \\
& =\left|\int_{\Omega}\left(H w-\frac{\mathrm{K}(\vartheta)}{\vartheta} \nabla \vartheta \cdot \nabla w-\frac{\mathrm{K}(\vartheta)}{\vartheta^{2}}|\nabla \vartheta|^{2} w+J w\right) \mathrm{d} x+\int_{\partial \Omega} h \frac{w}{\vartheta} \mathrm{d} S\right| \\
& \leq\left|\int_{\Omega} H w \mathrm{~d} x\right|+\left|\int_{\Omega} \frac{\mathrm{K}(\vartheta)}{\vartheta} \nabla \vartheta \cdot \nabla w \mathrm{~d} x\right|+\left.\left|\int_{\Omega} \frac{\mathrm{K}(\vartheta)}{\vartheta^{2}}\right| \nabla \vartheta\right|^{2} w \mathrm{~d} x|+| \int_{\Omega} J w \mathrm{~d} x|+| \int_{\partial \Omega} h \frac{w}{\vartheta} \mathrm{d} S \mid \\
& \doteq I_{1}+I_{2}+I_{3}+I_{4}+I_{5} .
\end{aligned}
$$

Estimate (3.19) yields that $\|H\|_{L^{2}\left(0, T ; L^{2}(\Omega)\right)} \leq C$, therefore $\left|I_{1}\right| \leq \mathcal{H}(t)\|w\|_{L^{2}(\Omega)}$ with $\mathcal{H}(t)=\|H(\cdot, t)\| L_{L^{2}(\Omega)} \in$ $L^{2}(0, T)$. Analogously, also in view of (2.24) and of (3.2) we have that

$$
\left|I_{4}\right| \leq \frac{1}{\vartheta_{*}} \mathcal{J}(t)\|w\|_{L^{\infty}(\Omega)} \quad \text { with } \mathcal{J}(t):=\|J(\cdot, t)\|_{L^{1}(\Omega)} \in L^{1}(0, T) .
$$

Moreover, $\left|I_{5}\right| \leq \frac{1}{\vartheta_{*}}\|h(t)\|_{L^{2}(\partial \Omega)}\|w\|_{L^{2}(\partial \Omega)}$, with $\|h(t)\|_{L^{2}(\partial \Omega)} \in L^{1}(0, T)$ thanks to (2.25). Using the growth condition (2.17) for $\mathrm{K}$, we estimate

$$
\left|I_{2}\right| \leq C \int_{\Omega} \vartheta^{\kappa-1}|\nabla \vartheta||\nabla w| \mathrm{d} x+C \int_{\Omega} \frac{1}{\vartheta}|\nabla \vartheta||\nabla w| \mathrm{d} x \doteq I_{2,1}+I_{2,2} .
$$

Thanks to the previously proved positivity (3.2), we have

$$
I_{2,2} \leq \frac{C}{\vartheta^{*}} \mathcal{O}(t)\|\nabla w\|_{L^{2}\left(\Omega ; \mathbb{R}^{d}\right)} \quad \text { with } \mathcal{O}(t):=\|\nabla \vartheta(t)\|_{L^{2}\left(\Omega ; \mathbb{R}^{d}\right)} \in L^{2}(0, T)
$$

by (3.14). We estimate $I_{2,1}$ via the Hölder inequality, taking into account (3.13) and (3.17), whence, for $d \in\{2,3\}$,

$$
\begin{gathered}
I_{2,1} \leq C\left\|\vartheta^{(\kappa+\alpha-2) / 2} \nabla \vartheta\right\|_{L^{2}\left(\Omega ; \mathbb{R}^{d}\right)}\left\|\vartheta^{(\kappa-\alpha) / 2}\right\|_{L^{6}(\Omega)}\|\nabla w\|_{L^{3}\left(\Omega ; \mathbb{R}^{d}\right)} \doteq C \mathcal{O}^{*}(t)\|\nabla w\|_{L^{3}\left(\Omega ; \mathbb{R}^{d}\right)} \\
\text { with } \mathcal{O}^{*}(t):=\left\|\vartheta(t)^{(\kappa+\alpha-2) / 2} \nabla \vartheta(t)\right\|_{L^{2}\left(\Omega ; \mathbb{R}^{d}\right)}\left\|\vartheta(t)^{(\kappa-\alpha) / 2}\right\|_{L^{6}(\Omega)} \in L^{1}(0, T)
\end{gathered}
$$


Finally, we have

$$
\left|I_{3}\right| \leq C \int_{\Omega} \vartheta^{\kappa-2}|\nabla \vartheta|^{2}|w| \mathrm{d} x+C \int_{\Omega} \frac{1}{\vartheta^{2}}|\nabla \vartheta|^{2}|w| \mathrm{d} x \doteq I_{3,1}+I_{3,2} .
$$

The positivity property (3.2) again guarantees

$$
I_{3,2} \leq \frac{C}{\vartheta_{*}^{2}} \mathcal{O}(t)^{2}\|w\|_{L^{\infty}(\Omega)} \quad \text { with } \mathcal{O}(t)^{2} \in L^{1}(0, T)
$$

while, using that $\vartheta^{\kappa-2} \leq c \vartheta^{\kappa+\alpha-2}+c^{\prime}$, we infer

$$
\begin{array}{r}
I_{3,2} \leq\|w\|_{L^{\infty}(\Omega)}\left(c \int_{\Omega} \vartheta^{\kappa+\alpha-2}|\nabla \vartheta|^{2} \mathrm{~d} x+c^{\prime} \int_{\Omega}|\nabla \vartheta|^{2} \mathrm{~d} x\right) \doteq\|w\|_{L^{\infty}(\Omega)} \mathcal{O}_{*}(t) \\
\text { with } \mathcal{O}_{*}(t)=c \int_{\Omega} \vartheta(t)^{\kappa+\alpha-2}|\nabla \vartheta(t)|^{2} \mathrm{~d} x+c^{\prime} \int_{\Omega}|\nabla \vartheta(t)|^{2} \mathrm{~d} x \in L^{1}(0, T),
\end{array}
$$

thanks to (3.13) and (3.14).

Collecting all of the above calculations, we conclude that

$$
\left\|\partial_{t} \log (\vartheta)\right\|_{L^{1}\left(0, T ;\left(W^{1, d}(\Omega) \cap L^{\infty}(\Omega)\right)^{*}\right)} \leq C .
$$

Seventh estimate $[\mu \in\{0,1\}], \kappa \in(1,5 / 3)$ if $d=3$ and $\kappa \in(1,2)$ if $d=2$. Assume in addition Hypothesis (V). We multiply (1.1) by a test function $w \in W^{1, \infty}(\Omega)$ (which e.g. holds if $w \in W^{2, d+\epsilon}(\Omega)$ for $\epsilon>0$ ). By comparison we have

$$
\left|\int_{\Omega} \vartheta_{t} w \mathrm{~d} x\right| \leq\left|\int_{\Omega} L w \mathrm{~d} x\right|+\left|\int_{\Omega} \mathrm{K}(\vartheta) \nabla \vartheta \cdot \nabla w \mathrm{~d} x\right|+\left|\int_{\partial \Omega} h w \mathrm{~d} S\right| \doteq I_{1}+I_{2}+I_{3},
$$

where we have set $L=-\chi_{t} \vartheta-\rho \vartheta \operatorname{div}\left(\mathbf{u}_{t}\right)+g+a(\chi) \varepsilon\left(\mathbf{u}_{t}\right) \mathbb{V} \varepsilon\left(\mathbf{u}_{t}\right)+\left|\chi_{t}\right|^{2}$. Therefore,

$$
\left|I_{1}\right| \leq \mathcal{L}(t)\|w\|_{L^{\infty}(\Omega)} \quad \text { with } \mathcal{L}(t):=\|L(t)\|_{L^{1}(\Omega)} \in L^{1}(0, T), \quad\left|I_{3}\right| \leq\|h(t)\|_{L^{2}(\partial \Omega)}\|w\|_{L^{2}(\partial \Omega)} \text { with } h \in L^{1}(0, T)
$$

thanks to (3.14), (3.19) and (2.25), respectively. As for $I_{2}$, in view of (2.17), taking into account (3.13) and using the Hölder inequality, we obtain

$$
\left|I_{2}\right| \leq C\left\|\vartheta^{(\kappa-\alpha+2) / 2}\right\|_{L^{2}(\Omega)}\left\|\vartheta^{(\kappa+\alpha-2) / 2} \nabla \vartheta\right\|_{L^{2}\left(\Omega ; \mathbb{R}^{d}\right)}\|\nabla w\|_{L^{\infty}\left(\Omega ; \mathbb{R}^{d}\right)}+C\|\nabla \vartheta\|_{L^{2}\left(\Omega ; \mathbb{R}^{d}\right)}\|\nabla w\|_{L^{2}\left(\Omega ; \mathbb{R}^{d}\right)} .
$$

Observe that, since $\alpha$ can be chosen arbitrarily close to 1 , in view of estimate (3.15) we have that $\vartheta^{(\kappa-\alpha+2) / 2}$ is bounded in $L^{2}\left(0, T ; L^{2}(\Omega)\right)$ if and only if $\kappa<\frac{5}{3}$ if $d=3$, and $\kappa<2$ if $d=2$. Under this restriction on $\kappa$, we have that $\left|I_{2}\right| \leq C \mathcal{L}^{*}(t)\|\nabla w\|_{L^{\infty}(\Omega)}$ for some $\mathcal{L}^{*} \in L^{1}(0, T)$. Ultimately, we conclude that

$$
\left\|\vartheta_{t}\right\|_{L^{1}\left(0, T ; W^{1, \infty}(\Omega)^{*}\right)} \leq C .
$$

Eighth estimate $[\mu=0]$. In view of the previously obtained estimates (3.5), (3.14), (3.19), and (3.26), a comparison in equation (1.3) yields that (recall that $\xi$ is a selection in $\beta(\chi)$ a.e. in $\Omega \times(0, T)$ ),

$$
\left\|A_{p}(\chi)+\xi\right\|_{L^{2}\left(0, T ; L^{2}(\Omega)\right)} \leq C .
$$

Now, in view of the monotonicity of the operator $\beta: \mathbb{R} \rightrightarrows \mathbb{R}$ (cf., e.g., [2, Lemma 3.3]), from the above estimate we deduce

$$
\left\|A_{p}(\chi)\right\|_{L^{2}\left(0, T ; L^{2}(\Omega)\right)}+\|\xi\|_{L^{2}\left(0, T ; L^{2}(\Omega)\right)} \leq C .
$$

In view of the regularity results [50, Thm. 2, Rmk. 2.5], we finally infer the enhanced regularity (2.55) for $\chi$.

Remark 3.1 (The $p$-Laplacian regularization). A close perusal at the above calculations shows that the fact that $p>d$ for the $p$-Laplacian term in the $\chi$-equation (1.3) has been used only for carrying out the calculations in the Fifth estimate. All the other estimates do not depend on the condition $p>d$, and would therefore hold if the operator $A_{p}$ in (1.3) were replaced by the Laplacian.

In turn, the Fifth estimate for $\mathbf{u}$ will play a crucial role in the limit passage arguments at the basis of the proofs of Theorems 1 and 2 it will ensure compactness in the strong topology of $H^{1}\left(0, T ; H_{\text {Dir }}^{2}\left(\Omega ; \mathbb{R}^{d}\right)\right.$ ) (cf. 
Lemma 5.1) for the sequences of approximate solutions constructed in Sec. 4. Relying on this, we will be able to pass to the limit with the quadratic term $\left|\varepsilon\left(\mathbf{u}_{t}\right)\right|^{2}$ on the right-hand side of (1.1).

Nonetheless, in Sec. 6 we will show that, in the case $\mu=1$ of unidirectional evolution, it is ultimately possible to drop the constraint $p>d$ and in fact we will obtain an existence result for the entropic formulation of system (1.1)-(1.3), in the case (1.3) simply features the Laplacian (i.e. for $p=2$ ).

\section{Time discretization}

In Section 4.1 we set up a single time-discretization scheme for both the irreversible $(\mu=1)$ and for the reversible $(\mu=0)$ systems. We then show in Section 4.2 that the piecewise constant and piecewise linear interpolants of the discrete solutions satisfy the approximate versions of the total energy inequality, the entropy inequality, and of equations (1.2)-(1.3). Finally, in Section 4.3 we rigorously prove the a priori estimates from Section 3 in the time-discrete context.

Notation 4.1. In what follows, also in view of the extension (2.52) mentioned at the end of Sec. 2.3, we will use $\widehat{\alpha}$ and $\alpha$ as place-holders for $I_{(-\infty, 0]}$ and $\partial I_{(-\infty, 0]}$.

4.1. Setup of the time discretization. We consider an equidistant partition of $[0, T]$, with time-step $\tau>0$ and nodes $t_{\tau}^{k}:=k \tau, k=0, \ldots, K_{\tau}$. In this framework, we approximate the data $\mathbf{f}, g$, and $h$ by local means, i.e. setting for all $k=1, \ldots, K_{\tau}$

$$
\mathbf{f}_{\tau}^{k}:=\frac{1}{\tau} \int_{t_{\tau}^{k-1}}^{t_{\tau}^{k}} \mathbf{f}(s) \mathrm{d} s, \quad g_{\tau}^{k}:=\frac{1}{\tau} \int_{t_{\tau}^{k-1}}^{t_{\tau}^{k}} g(s) \mathrm{d} s, \quad h_{\tau}^{k}:=\frac{1}{\tau} \int_{t_{\tau}^{k-1}}^{t_{\tau}^{k}} h(s) \mathrm{d} s .
$$

Consider the following initial data

$$
\vartheta_{\tau}^{0}:=\vartheta_{0}, \quad \mathbf{u}_{\tau}^{0}:=\mathbf{u}_{0}, \quad \mathbf{u}_{\tau}^{-1}:=\mathbf{u}_{0}-\tau \mathbf{v}_{0}, \quad \chi_{\tau}^{0}:=\chi_{0} .
$$

We construct discrete solutions to system (1.1)-(1.3) by solving the following elliptic system, featuring the operator $\mathcal{A}^{k}: X \rightarrow H^{1}(\Omega)^{*}$, with

$$
\begin{gathered}
X=\left\{\theta \in H^{1}(\Omega): \int_{\Omega} \mathrm{K}(\theta) \nabla \theta \cdot \nabla v \mathrm{~d} x \text { is well defined for all } v \in H^{1}(\Omega)\right\}, \quad \mathcal{A}^{k}: X \rightarrow H^{1}(\Omega)^{*} \text { defined by } \\
\left\langle\left\langle\mathcal{A}^{k}(\theta), v\right\rangle_{H^{1}(\Omega)}:=\int_{\Omega} \mathrm{K}(\theta) \nabla \theta \cdot \nabla v \mathrm{~d} x-\int_{\partial \Omega} h_{\tau}^{k} v \mathrm{~d} S .\right.
\end{gathered}
$$

Problem 4.2. Starting from $\left(\mathbf{u}_{\tau}^{0}, \mathbf{u}_{\tau}^{-1}, \chi_{\tau}^{0}, \vartheta_{\tau}^{0}\right)$ as in (4.2), find $\left\{\vartheta_{\tau}^{k}, \mathbf{u}_{\tau}^{k}, \chi_{\tau}^{k}\right\}_{k=1}^{K_{\tau}} \subset X \times H_{\text {Dir }}^{2}\left(\Omega ; \mathbb{R}^{d}\right) \times W^{1, p}(\Omega)$ fulfilling

$$
\begin{aligned}
& \frac{\vartheta_{\tau}^{k}-\vartheta_{\tau}^{k-1}}{\tau}+\frac{\chi_{\tau}^{k}-\chi_{\tau}^{k-1}}{\tau} \vartheta_{\tau}^{k}+\rho \operatorname{div}\left(\frac{\mathbf{u}_{\tau}^{k}-\mathbf{u}_{\tau}^{k-1}}{\tau}\right) \vartheta_{\tau}^{k}+\mathcal{A}^{k}\left(\vartheta_{\tau}^{k}\right)=g_{\tau}^{k} \\
& \quad+a\left(\chi_{\tau}^{k-1}\right) \varepsilon\left(\frac{\mathbf{u}_{\tau}^{k}-\mathbf{u}_{\tau}^{k-1}}{\tau}\right) \mathbb{V} \varepsilon\left(\frac{\mathbf{u}_{\tau}^{k}-\mathbf{u}_{\tau}^{k-1}}{\tau}\right)+\left|\frac{\chi_{\tau}^{k}-\chi_{\tau}^{k-1}}{\tau}\right|^{2}+\frac{\tau^{1 / 2}}{2}\left|\frac{\chi_{\tau}^{k}-\chi_{\tau}^{k-1}}{\tau}\right|^{2} \quad \text { in } H^{1}(\Omega)^{*} \\
& \frac{\mathbf{u}_{\tau}^{k}-2 \mathbf{u}_{\tau}^{k-1}+\mathbf{u}_{\tau}^{k-2}}{\tau^{2}}+\nu\left(a\left(\chi_{\tau}^{k-1}\right) \frac{\mathbf{u}_{\tau}^{k}-\mathbf{u}_{\tau}^{k-1}}{\tau}\right)+\varepsilon\left(b\left(\chi_{\tau}^{k}\right) \mathbf{u}_{\tau}^{k}\right)+\mathcal{C}_{\rho}\left(\vartheta_{\tau}^{k}\right)=\mathbf{f}_{\tau}^{k} \quad \text { a.e. in } \Omega \\
& \frac{\chi_{\tau}^{k}-\chi_{\tau}^{k-1}}{\tau}+\sqrt{\tau} \frac{\chi_{\tau}^{k}-\chi_{\tau}^{k-1}}{\tau}+\mu \zeta_{\tau}^{k}+A_{p}\left(\chi_{\tau}^{k}\right)+\xi_{\tau}^{k}+\gamma\left(\chi_{\tau}^{k}\right) \ni-b^{\prime}\left(\chi_{\tau}^{k}\right) \frac{\varepsilon\left(\mathbf{u}_{\tau}^{k-1}\right) \mathbb{E} \varepsilon\left(\mathbf{u}_{\tau}^{k-1}\right)}{2}+\vartheta_{\tau}^{k} \quad \text { a.e. in } \Omega
\end{aligned}
$$

where $\mathbb{I} \in \mathbb{R}^{d \times d \times d \times d}$ denotes the identity tensor and

$$
\begin{array}{ll}
\xi_{\tau}^{k} \in \beta\left(\chi_{\tau}^{k}\right) & \text { a.e. in } \Omega, \\
\zeta_{\tau}^{k} \in \alpha\left(\frac{\chi_{\tau}^{k}-\chi_{\tau}^{k-1}}{\tau}\right) & \text { a.e. in } \Omega .
\end{array}
$$


Remark 4.3 (Features of the time-discretization scheme). A few observations on Problem 4.2 are in order.

First of all, let us point out that the scheme is fully implicit and, in particular, (4.6) is coupled to the system (4.4) - 4.5) by the implicit term $\vartheta_{\tau}^{k}$ on the right-hand side. This will be crucial for proving the strict positivity (4.10) below for the discrete temperature $\vartheta_{\tau}^{k}$. Indeed, our argument for (4.10) is the discrete version of the comparison argument developed at the beginning of Section 3 and strongly relies on the structure of the discrete temperature equation (4.4). However, in the case of unidirectional evolution, we could have decoupled the discrete equation for $\chi$ from (4.4)-(4.5), replacing (4.6) by

$$
\frac{\chi_{\tau}^{k}-\chi_{\tau}^{k-1}}{\tau}+\mu \zeta_{\tau}^{k}+A_{p}\left(\chi_{\tau}^{k}\right)+\xi_{\tau}^{k}+\gamma\left(\chi_{\tau}^{k}\right) \ni-b^{\prime}\left(\chi_{\tau}^{k}\right) \frac{\varepsilon\left(\mathbf{u}_{\tau}^{k-1}\right) \mathbb{E} \varepsilon\left(\mathbf{u}_{\tau}^{k-1}\right)}{2}+\vartheta_{\tau}^{k-1} \quad \text { a.e. in } \Omega,
$$

and, accordingly, replacing the coupling term $\frac{\chi_{\tau}^{k}-\chi_{\tau}^{k-1}}{\tau} \vartheta_{\tau}^{k}$ on the left-hand side of (4.4) by $\frac{\chi_{\tau}^{k}-\chi_{\tau}^{k-1}}{\tau} \vartheta_{\tau}^{k-1}$. In Remark 4.5 below, we will show how it is still possible to prove the strict positivity of the discrete temperature for this partially decoupled scheme.

Second, observe that $\frac{\tau^{1 / 2}}{2}\left|\frac{\chi_{\tau}^{k}-\chi_{\tau}^{k-1}}{\tau}\right|^{2}$ appears on the right-hand side of (4.4) and, accordingly, $\sqrt{\tau} \frac{\chi_{\tau}^{k}-\chi_{\tau}^{k-1}}{\tau}$ features on the left-hand side of (4.6). These terms have been added for technical reasons, related to the proof of the discrete version of the total energy inequality (2.40), cf. the comments above Proposition 4.8. Clearly, they will disappear when passing to the limit with $\tau \downarrow 0$.

Because of the implicit character of system (4.4)-(4.6), for the existence proof (cf. Lemma 4.4 below) we shall have to resort to a fixed-point type result from the theory for elliptic systems featuring pseudo-monotone operators, drawn from [48, Chap. II]. Indeed, we will not apply it directly to system (4.4)-(4.6), but to an approximation of (4.4)-(4.6), i.e. system (4.15) (4.17) below, obtained in the following way. We will need to

(1) truncate $\mathrm{K}$, along the lines of [23], in such a way as to have a bounded function in the elliptic operator in the temperature equation (4.4). Therefore, the truncated operator $\mathrm{K}_{M}$, with $M$ a positive parameter, shall be defined on $H^{1}(\Omega)$ (in place of $X$ ), with values in $H^{1}(\Omega)^{*}$ (in place of $X^{*}$ ). Accordingly, we shall truncate all occurrences of $\vartheta$ in a quadratic term;

(2) following [49], add the higher order terms $-\nu \operatorname{div}\left(\left|\varepsilon\left(\mathbf{u}_{\tau}^{k}\right)\right|^{\eta-2} \mathbb{I} \varepsilon\left(\mathbf{u}_{\tau}^{k}\right)\right)$ and $\nu\left|\chi_{\tau}^{k}\right|^{\eta-2} \chi_{\tau}^{k}$, with $\nu>0$ and $\eta>4$, on the left-hand sides of (4.5) and (4.6), respectively. Their role is to compensate the quadratic terms on the right-hand side of (4.4). As a result, both for $d=2$ and for $d=3$ the pseudo-monotone operator by means of which we will rephrase system 4.15) (4.17) will turn out to be coercive, in its $\vartheta$-component, with respect to the $H^{1}(\Omega)$-norm;

(3) in the case $\mu=1$, in order to cope with the (possible) unboundedness of the operator $\alpha$ we will have to replace it with its Yosida-regularization $\alpha_{\nu}$ (cf. 9] ), with $\nu$ the same parameter as above.

Then, in the proof of Lemma 4.4 we will

(1) prove the existence of solutions to the approximate discrete system (4.15)-(4.17);

(2) pass to the limit in (4.15)-(4.17) first as the truncation parameter $M \rightarrow \infty$ and conclude an existence result for an approximation of system (4.4)-(4.6), still depending on the parameter $\nu>0$;

(3) pass to the limit in this approximate system as $\nu \rightarrow 0$ and conclude the existence of solutions to (4.4)- (4.6)

We postpone to Remark 4.6 some comments on the reason why we need to keep the two limit passages as $M \rightarrow \infty$ and $\nu \rightarrow 0$ distinct.

Our existence result for Problem 4.2 reads

Lemma 4.4 (Existence for the time-discrete Problem 4.2, $\mu \in\{0,1\}$ ). Assume Hypotheses (O)-(III), and assumptions (2.23) -(2.28) on the data $\mathbf{f}, g, h, \vartheta_{0}, \mathbf{u}_{0}, \mathbf{v}_{0}, \chi_{0}$. Then, there exists $\bar{\tau}>0$ such that for all $0<\tau \leq \bar{\tau}$ Problem 4.2, admits at least one solution $\left\{\left(\vartheta_{\tau}^{k}, \mathbf{u}_{\tau}^{k}, \chi_{\tau}^{k}\right)\right\}_{k=1}^{K_{\tau}}$.

Furthermore, any solution $\left\{\left(\vartheta_{\tau}^{k}, \mathbf{u}_{\tau}^{k}, \chi_{\tau}^{k}\right)\right\}_{k=1}^{K_{\tau}}$ of Problem 4.2 fulfills

$$
\vartheta_{\tau}^{k}(x) \geq \underline{\vartheta}>0 \quad \text { for a.a. } x \in \Omega
$$


for some $\underline{\vartheta}=\underline{\vartheta}(T)$.

Proof. We split the proof in some steps.

Step 1: approximation. As already mentioned, we construct our approximation of system (4.4)-(4.6) by truncating $\mathrm{K}$ in (4.4) and the quadratic terms in $\vartheta$, replacing $\alpha$ with its Yosida approximation $\alpha_{\nu}$, and adding higher order terms to (4.5) and (4.6). Namely, let

$$
\mathrm{K}_{M}(r):= \begin{cases}\mathrm{K}(-M) & \text { if } r<-M \\ \mathrm{~K}(r) & \text { if }|r| \leq M, \\ \mathrm{~K}(M) & \text { if } r>M\end{cases}
$$

and accordingly introduce the operator

$$
\mathcal{A}_{M}^{k}: H^{1}(\Omega) \rightarrow H^{1}(\Omega)^{*} \text { defined by }\left\langle\mathcal{A}_{M}^{k}(\theta), v\right\rangle_{H^{1}(\Omega)}:=\int_{\Omega} \mathrm{K}_{M}(\theta) \nabla \theta \cdot \nabla v \mathrm{~d} x-\int_{\partial \Omega} h_{\tau}^{k} v \mathrm{~d} S .
$$

Observe that, thanks to (2.17) there still holds $\mathrm{K}_{M}(r) \geq c_{0}$ for all $r \in \mathbb{R}$, and therefore

$$
\left\langle\mathcal{A}_{M}^{k}(\theta), \theta\right\rangle_{H^{1}(\Omega)} \geq c_{0} \int_{\Omega}|\nabla \theta|^{2} \mathrm{~d} x \quad \text { for all } \theta \in H^{1}(\Omega) .
$$

We also introduce the truncation operator $\mathcal{T}_{M}: \mathbb{R} \rightarrow \mathbb{R}$

$$
\mathcal{T}_{M}(r):= \begin{cases}-M & \text { if } r<-M \\ r & \text { if }|r| \leq M \\ M & \text { if } r>M\end{cases}
$$

Furthermore, for a given $\nu>0$ we denote by $\alpha_{\nu}$ the Yosida approximation of $\alpha$ with parameter $\nu$.

Then, we consider the following approximation of system (4.4)-(4.6):

$$
\begin{aligned}
& \frac{\vartheta_{\tau}^{k}-\vartheta_{\tau}^{k-1}}{\tau}+\frac{\chi_{\tau}^{k}-\chi_{\tau}^{k-1}}{\tau} \mathcal{T}_{M}\left(\vartheta_{\tau}^{k}\right)+\rho \operatorname{div}\left(\frac{\mathbf{u}_{\tau}^{k}-\mathbf{u}_{\tau}^{k-1}}{\tau}\right) \mathcal{T}_{M}\left(\vartheta_{\tau}^{k}\right)+\mathcal{A}_{M}^{k}\left(\vartheta_{\tau}^{k}\right)=g_{\tau}^{k} \\
& \quad+a\left(\chi_{\tau}^{k-1}\right) \varepsilon\left(\frac{\mathbf{u}_{\tau}^{k}-\mathbf{u}_{\tau}^{k-1}}{\tau}\right) \mathbb{V} \varepsilon\left(\frac{\mathbf{u}_{\tau}^{k}-\mathbf{u}_{\tau}^{k-1}}{\tau}\right)+\left|\frac{\chi_{\tau}^{k}-\chi_{\tau}^{k-1}}{\tau}\right|^{2}+\frac{\tau^{1 / 2}}{2}\left|\frac{\chi_{\tau}^{k}-\chi_{\tau}^{k-1}}{\tau}\right|^{2} \quad \text { in } H^{1}(\Omega)^{*}, \\
& \frac{\mathbf{u}_{\tau}^{k}-2 \mathbf{u}_{\tau}^{k-1}+\mathbf{u}_{\tau}^{k-2}}{\tau^{2}}+\mathcal{V}\left(a\left(\chi_{\tau}^{k-1}\right) \frac{\mathbf{u}_{\tau}^{k}-\mathbf{u}_{\tau}^{k-1}}{\tau}\right)+\varepsilon\left(b\left(\chi_{\tau}^{k}\right) \mathbf{u}_{\tau}^{k}\right)+\mathcal{C}_{\rho}\left(\mathcal{T}_{M}\left(\vartheta_{\tau}^{k}\right)\right)-\nu \operatorname{div}\left(\left|\varepsilon\left(\mathbf{u}_{\tau}^{k}\right)\right|^{\eta-2} \mathbb{I} \varepsilon\left(\mathbf{u}_{\tau}^{k}\right)\right)=\mathbf{f}_{\tau}^{k} \\
& \text { in } W_{0}^{1, \eta}\left(\Omega ; \mathbb{R}^{d}\right)^{*},
\end{aligned}
$$

$$
\begin{array}{r}
\frac{\chi_{\tau}^{k}-\chi_{\tau}^{k-1}}{\tau}+\sqrt{\tau} \frac{\chi_{\tau}^{k}-\chi_{\tau}^{k-1}}{\tau}+\mu \alpha_{\nu}\left(\frac{\chi_{\tau}^{k}-\chi_{\tau}^{k-1}}{\tau}\right)+A_{p}\left(\chi_{\tau}^{k}\right)+\xi_{\tau}^{k}+\gamma\left(\chi_{\tau}^{k}\right)+\nu\left|\chi_{\tau}^{k}\right|^{\eta-2} \chi_{\tau}^{k} \\
=-b^{\prime}\left(\chi_{\tau}^{k}\right) \frac{\varepsilon\left(\mathbf{u}_{\tau}^{k-1}\right) \mathbb{E} \varepsilon\left(\mathbf{u}_{\tau}^{k-1}\right)}{2}+\mathcal{T}_{M}\left(\vartheta_{\tau}^{k}\right) \quad \text { a.e. in } \Omega,
\end{array}
$$

with $\xi_{\tau}^{k} \in \beta\left(\chi_{\tau}^{k}\right)$ a.e. in $\Omega$. 
Step 2: existence of solutions for the approximate system. Observe that system (4.15)-4.17) can be recast as

$$
\begin{aligned}
& \vartheta_{\tau}^{k}+\left(\chi_{\tau}^{k}-\chi_{\tau}^{k-1}\right) \mathcal{T}_{M}\left(\vartheta_{\tau}^{k}\right)+\rho \operatorname{div}\left(\mathbf{u}_{\tau}^{k}-\mathbf{u}_{\tau}^{k-1}\right) \mathcal{T}_{M}\left(\vartheta_{\tau}^{k}\right)+\tau \mathcal{A}_{M}^{k}\left(\vartheta_{\tau}^{k}\right) \\
& -\tau a\left(\chi_{\tau}^{k-1}\right) \varepsilon\left(\frac{\mathbf{u}_{\tau}^{k}-\mathbf{u}_{\tau}^{k-1}}{\tau}\right) \mathbb{V} \varepsilon\left(\frac{\mathbf{u}_{\tau}^{k}-\mathbf{u}_{\tau}^{k-1}}{\tau}\right)-\tau\left|\frac{\chi_{\tau}^{k}-\chi_{\tau}^{k-1}}{\tau}\right|^{2}-\frac{\tau^{3 / 2}}{2}\left|\frac{\chi_{\tau}^{k}-\chi_{\tau}^{k-1}}{\tau}\right|^{2} \\
& =\vartheta_{\tau}^{k-1}+\tau g_{\tau}^{k} \text { in } H^{1}(\Omega)^{*}, \\
& \mathbf{u}_{\tau}^{k}+\tau \mathcal{V}\left(a\left(\chi_{\tau}^{k-1}\right)\left(\mathbf{u}_{\tau}^{k}-\mathbf{u}_{\tau}^{k-1}\right)\right)+\tau^{2} \mathcal{E}\left(b\left(\chi_{\tau}^{k}\right) \mathbf{u}_{\tau}^{k}\right)+\tau^{2} \mathcal{C}_{\rho}\left(\mathcal{T}_{M}\left(\vartheta_{\tau}^{k}\right)\right)-\nu \tau^{2} \operatorname{div}\left(\left|\varepsilon\left(\mathbf{u}_{\tau}^{k}\right)\right|^{\eta-2} \mathbb{I} \varepsilon\left(\mathbf{u}_{\tau}^{k}\right)\right) \\
& =2 \mathbf{u}_{\tau}^{k-1}-\mathbf{u}_{\tau}^{k-2}+\tau^{2} \mathbf{f}_{\tau}^{k} \quad \text { in } W_{0}^{1, \eta}\left(\Omega ; \mathbb{R}^{d}\right)^{*}, \\
& \chi_{\tau}^{k}+\sqrt{\tau} \chi_{\tau}^{k}+\mu \tau \alpha_{\nu}\left(\frac{\chi_{\tau}^{k}-\chi_{\tau}^{k-1}}{\tau}\right)+\tau A_{p}\left(\chi_{\tau}^{k}\right) \\
& +\tau \xi_{\tau}^{k}+\tau \gamma\left(\chi_{\tau}^{k}\right)+\nu \tau\left|\chi_{\tau}^{k}\right|^{\eta-2} \chi_{\tau}^{k}+\tau b^{\prime}\left(\chi_{\tau}^{k}\right) \frac{\varepsilon\left(\mathbf{u}_{\tau}^{k-1}\right) \mathbb{E} \varepsilon\left(\mathbf{u}_{\tau}^{k-1}\right)}{2}-\tau \mathcal{T}_{M}\left(\vartheta_{\tau}^{k}\right) \\
& =\chi_{\tau}^{k-1}+\sqrt{\tau} \chi_{\tau}^{k-1} \text { a.e. in } \Omega \text {. }
\end{aligned}
$$

Denoting by $\mathcal{R}_{k-1}$ the operator acting on the unknown $\left(\vartheta_{\tau}^{k}, \mathbf{u}_{\tau}^{k}, \chi_{\tau}^{k}\right)$ and by $H_{k-1}$ the vector of the terms on the r.h.s. of the above equations, we can reformulate system (4.18)-(4.20) in the abstract form

$$
\mathcal{R}_{k-1}\left(\vartheta_{\tau}^{k}, \mathbf{u}_{\tau}^{k}, \chi_{\tau}^{k}\right)=H_{k-1} .
$$

It can be checked that $\mathcal{R}_{k-1}$ is a pseudo-monotone operator (according to [48, Chap. II, Def. 2.1]) on $H^{1}(\Omega) \times W_{0}^{1, \eta}\left(\Omega ; \mathbb{R}^{d}\right) \times H^{1}(\Omega)$.

In order to check that $\mathcal{R}_{k-1}$ is coercive on that space, it is sufficient to test (4.18) by $\vartheta_{\tau}^{k}$, (4.19) by $\mathbf{u}_{\tau}^{k}$, (4.20) by $\chi_{\tau}^{k}$ and add the resulting equations. We will not develop all the calculations in full detail, but rather point to the most significant aspects.

Clearly, the term $-\nu \tau^{2} \operatorname{div}\left(\left|\varepsilon\left(\mathbf{u}_{\tau}^{k}\right)\right|^{\eta-2} \mathbb{I} \varepsilon\left(\mathbf{u}_{\tau}^{k}\right)\right)$ tested by $\mathbf{u}_{\tau}^{k}$ provides a bound for $\left\|\mathbf{u}_{\tau}^{k}\right\|_{W^{1, \gamma}\left(\Omega ; \mathbb{R}^{d}\right)}^{\gamma}$ via the Korn inequality. Analogously we control $\left\|\chi_{\tau}^{k}\right\|_{H^{1}(\Omega)}^{2}$. To obtain a bound for $\left\|\vartheta_{\tau}^{k}\right\|_{H^{1}(\Omega)}$ we use that $\mathcal{A}_{M}^{k}$ is coercive (cf. (4.13)). The additional terms $-\nu \operatorname{div}\left(\left|\varepsilon\left(\mathbf{u}_{\tau}^{k}\right)\right|^{\eta-2} \mathbb{I} \varepsilon\left(\mathbf{u}_{\tau}^{k}\right)\right)$ and $\nu\left|\chi_{\tau}^{k}\right|^{\eta-2} \chi_{\tau}^{k}$ in (4.19) and (4.20) enable us to control the quadratic terms on the right-hand side of (4.18). More in detail, the test of (4.18) by $\vartheta_{\tau}^{k}$ gives rise, e.g., to the term $I_{1}:=\int_{\Omega} a\left(\chi_{\tau}^{k-1}\right) \varepsilon\left(\mathbf{u}_{\tau}^{k}\right) \mathbb{V} \varepsilon\left(\mathbf{u}_{\tau}^{k}\right) \vartheta_{\tau}^{k} \mathrm{~d} x$, which can be estimated as follows

$$
\begin{aligned}
\left|I_{1}\right| & \leq C\left\|a\left(\chi_{\tau}^{k-1}\right)\right\|_{L^{\infty}(\Omega)}\left\|\varepsilon\left(\mathbf{u}_{\tau}^{k}\right)\right\|_{L^{4}\left(\Omega ; \mathbb{R}^{d \times d}\right)}^{2}\left\|\vartheta_{\tau}^{k}\right\|_{L^{2}(\Omega)} \\
& \leq \frac{1}{4}\left\|\vartheta_{\tau}^{k}\right\|_{L^{2}(\Omega)}^{2}+C\left\|\varepsilon\left(\mathbf{u}_{\tau}^{k}\right)\right\|_{L^{4}\left(\Omega ; \mathbb{R}^{d \times d}\right)}^{4} \\
& \leq \frac{1}{4}\left\|\vartheta_{\tau}^{k}\right\|_{L^{2}(\Omega)}^{2}+\frac{\nu \tau^{2}}{4}\left\|\varepsilon\left(\mathbf{u}_{\tau}^{k}\right)\right\|_{L^{\eta}\left(\Omega ; \mathbb{R}^{d \times d}\right)}^{\eta}+C
\end{aligned}
$$

where the first estimate follows from the Hölder inequality, the second one from the fact that $\left\|a\left(\chi_{\tau}^{k-1}\right)\right\|_{L^{\infty}(\Omega)} \leq$ $C$ since $\chi_{\tau}^{k-1} \in W^{1, p}(\Omega)$ and $a \in \mathrm{C}^{0}(\mathbb{R})$, and the last one relies on the fact that $\eta>4$. Therefore, for $\tau$ sufficiently small the right-hand side terms can be absorbed by the left-hand side ones, also resulting from the test of (4.19) by $\mathbf{u}_{\tau}^{k}$. With analogous calculations we estimate $I_{2}:=\int_{\Omega}\left(\left|\chi_{\tau}^{k}\right|^{2}+\frac{\tau^{1 / 2}}{2}\left|\chi_{\tau}^{k}\right|^{2}\right) \vartheta_{\tau}^{k} \mathrm{~d} x$, exploiting the term $\nu \tau\left|\chi_{\tau}^{k}\right|^{\eta-2} \chi_{\tau}^{k}$ on the left-hand side of (4.20) which, tested by $\chi_{\tau}^{k}$, gives $\int_{\Omega}\left|\chi_{\tau}^{k}\right|^{\eta} \mathrm{d} x$ on the left-hand side.

Since $\mathcal{R}_{k-1}$ is pseudo-monotone and coercive, the Leray-Lions type existence result of [48, Chap. II, Thm. 2.6] applies, yielding the existence of a solution $\left(\vartheta_{\tau}^{k}, \mathbf{u}_{\tau}^{k}, \chi_{\tau}^{k}\right)$ (whose dependence on the parameters $M$ and $\nu$ is not highlighted, for simplicity) to (4.15)-4.17).

Step 3: proof of the strict positivity (4.10). Observe first that, for $\vartheta_{\tau}^{k}$ solving (4.15)-(4.17) the strict positivity (4.10) holds for $k=0$ with $\underline{\vartheta}:=\vartheta_{*}$ due to (2.26). In order to prove that $\vartheta_{\tau}^{k} \geq \underline{\vartheta}>0$ a.e. in $\Omega$, for every $k \geq 1$, we proceed in the same spirit of the proof of the strict positivity of $\vartheta$ in Sec. 3] (cf. also [31, Sec. $5.2])$. Namely, we start by deducing from (4.4) that

$$
\int_{\Omega} \frac{\vartheta_{\tau}^{k}-\vartheta_{\tau}^{k-1}}{\tau} w \mathrm{~d} x+\int_{\Omega} \mathrm{K}_{M}\left(\vartheta_{\tau}^{k}\right) \nabla \vartheta_{\tau}^{k} \nabla w \mathrm{~d} x \geq-C \int_{\Omega}\left(\vartheta_{\tau}^{k}\right)^{2} w \mathrm{~d} x \quad \text { for every } w \in W_{+}^{1,2}(\Omega)
$$


(cf. (2.1) for $W_{+}^{1,2}(\Omega)$ ), where $C$ is independent of $k$. We now consider the decreasing sequence $\left\{v_{k}\right\} \subseteq \mathbb{R}$ defined recursively as

$$
\frac{v_{k}-v_{k-1}}{\tau}=-C v_{k}^{2}, \quad v_{0}=\vartheta_{*}>0
$$

where $C$ is the same constant of (4.22). We write now (4.23), adding the term $-\operatorname{div}\left(\mathrm{K}_{M}\left(\vartheta_{\tau}^{k}\right) \nabla v_{k}\right)=0$, in the form

$$
\frac{1}{\tau} \int_{\Omega}\left(v_{k}-v_{k-1}\right) w \mathrm{~d} x+\int_{\Omega} \mathrm{K}_{M}\left(\vartheta_{\tau}^{k}\right) \nabla v_{k} \cdot \nabla w \mathrm{~d} x=-C \int_{\Omega} v_{k}^{2} w \mathrm{~d} x \quad \text { for every } w \in W_{+}^{1,2}(\Omega) .
$$

Subtracting (4.22) from (4.23) and testing the difference by $w=H_{\varepsilon}\left(v_{k}-\vartheta_{k}\right)$, where

$$
H_{\varepsilon}(v)= \begin{cases}0 & \text { if } v \leq 0 \\ v / \varepsilon & \text { if } v \in(0, \varepsilon) \\ 1 & \text { if } v \geq \varepsilon\end{cases}
$$

we obtain, since $v_{k}<v_{k-1}$ that

$$
\int_{\Omega}\left(\left(v_{k}-v_{k-1}\right)-\left(\vartheta_{\tau}^{k}-\vartheta_{\tau}^{k-1}\right)\right) H_{\varepsilon}\left(v_{k}-\vartheta_{\tau}^{k}\right) \mathrm{d} x \leq 0 .
$$

Assume now that $\vartheta_{\tau}^{k-1} \geq v_{k-1}$ a.e. in $\Omega$ (which is true for $k=1$ ). Taking $\varepsilon \searrow 0$, (4.24) yields $\vartheta_{\tau}^{k} \geq v_{k}$ a.e. in $\Omega$, and, by induction, $\vartheta_{\tau}^{k} \geq v_{k}>v_{K_{\tau}}$ a.e. in $\Omega$ for every $k=1, \ldots, K_{\tau}$. We now prove that there exists $\underline{\vartheta}>0$ such that $v_{K_{\tau}} \geq \underline{\vartheta}$ a.e. in $\Omega$. To this aim, observe that $v_{K_{\tau}}$ rewrites as $v_{K_{\tau}}=G^{-1}\left(G\left(v_{K_{\tau}}\right)\right)$, where $G(z):=-\int_{z}^{v_{0}} \frac{1}{s^{2}} \mathrm{~d} s$ is monotonically increasing on $\left(0, v_{0}\right], G(0+)=-\infty, G\left(v_{0}\right)=0$, hence, by the mean value theorem, for every $k=1, \ldots, K_{\tau}$ there exists $s_{k} \in\left[v_{k}, v_{k-1}\right]$ such that

$$
\frac{G\left(v_{k}\right)-G\left(v_{k-1}\right)}{v_{k}-v_{k-1}}=G^{\prime}\left(s_{k}\right)=\frac{1}{s_{k}^{2}} \leq \frac{1}{v_{k}^{2}},
$$

from which we deduce, using (4.23),

$$
\frac{G\left(v_{k}\right)-G\left(v_{k-1}\right)}{-C \tau v_{k}^{2}} \leq \frac{1}{v_{k}^{2}} \Longrightarrow G\left(v_{K_{\tau}}\right) \geq-C \tau K_{\tau},
$$

where the implication is also due to the fact that $G\left(v_{0}\right)=0$. Hence, we get

$$
\vartheta_{\tau}^{k}>v_{K_{\tau}}=G^{-1}\left(G\left(v_{K_{\tau}}\right)\right) \geq G^{-1}\left(-C \tau K_{\tau}\right)=G^{-1}(-C T)=: \underline{\vartheta}(T) .
$$

Thus, we conclude (4.10) with $\underline{\vartheta}=G^{-1}(-C T)$.

Step 4: passage to the limit as $M \rightarrow \infty$. We now pass to the limit in (4.15)-(4.17) as $M \rightarrow \infty$, for $\nu>0$ fixed. In this framework, we will denote by $\left(\vartheta_{M}, \mathbf{u}_{M}, \chi_{M}\right)$ the solutions of (4.15)-(4.17), with $\left(\vartheta_{\tau}^{k-1}, \mathbf{u}_{\tau}^{k-1}, \chi_{\tau}^{k-1}\right)$ given and $\nu>0$ fixed. First of all, we derive a bunch of estimates for $\left(\vartheta_{M}, \mathbf{u}_{M}, \chi_{M}\right)_{M}$, holding for constants independent of $M>0$ (but possibly depending on $\tau>0$, as well as on norms of $\left(\vartheta_{\tau}^{k-1}, \mathbf{u}_{\tau}^{k-1}, \chi_{\tau}^{k-1}\right)$ ).

We test (4.15) by 1 , (4.16) by $\frac{\mathbf{u}_{M}-\mathbf{u}_{\tau}^{k-1}}{\tau}$, (4.17) by $\frac{\chi_{M}-\chi_{\tau}^{k-1}}{\tau}$, and add the resulting relations. Taking into account all cancellations, conditions (2.23) $-(2.28)$, as well as the fact that the Yosida approximation $\widehat{\alpha}_{\nu}$ of $\widehat{\alpha}=I_{(\infty, 0]}$ is a positive function, we obtain that

$$
\exists C>0 \forall M>0: \quad\left\|\vartheta_{M}\right\|_{L^{1}(\Omega)}+\left\|\mathbf{u}_{M}\right\|_{H^{1}\left(\Omega ; \mathbb{R}^{d}\right)}+\nu^{1 / \eta}\left\|\varepsilon\left(\mathbf{u}_{M}\right)\right\|_{L^{\eta}\left(\Omega ; \mathbb{R}^{d \times d}\right)}+\left\|\chi_{M}\right\|_{W^{1, p}(\Omega)} \leq C .
$$

We now introduce the notation

$$
\mathcal{S}_{M}:=\left\{x \in \Omega: \vartheta_{M}(x) \leq M\right\}, \quad \mathcal{O}_{M}:=\Omega \backslash \mathcal{S}_{M} .
$$

In view of Markov's inequality and of estimate (4.26), we have that

$$
\left|\mathcal{O}_{M}\right| \leq \int_{\mathcal{O}_{M}} \frac{\vartheta_{M}}{M} \mathrm{~d} x \leq \frac{1}{M}\left\|\vartheta_{M}\right\|_{L^{1}(\Omega)} \leq \frac{C}{M} \rightarrow 0 \text { as } M \rightarrow \infty .
$$


We now test (4.15) by $\mathcal{T}_{M}\left(\vartheta_{M}\right)$. Observing that

$$
\left.\begin{array}{l}
\left.\mathrm{K}_{M}\left(\vartheta_{M}\right) \nabla \vartheta_{M} \nabla\left(\mathcal{T}_{M}\left(\vartheta_{M}\right)\right)=\mathrm{K}\left(\mathcal{T}_{M}\left(\vartheta_{M}\right)\right) \mid \nabla\left(\mathcal{T}_{M}\left(\vartheta_{M}\right)\right)\right)\left.\right|^{2} \\
\vartheta_{M} \mathcal{T}_{M}\left(\vartheta_{M}\right) \geq\left|\mathcal{T}_{M}\left(\vartheta_{M}\right)\right|^{2}
\end{array}\right\} \quad \text { a.e. in } \Omega
$$

we get

$$
\begin{aligned}
\left.\frac{1}{\tau} \int_{\Omega}\left|\mathcal{T}_{M}\left(\vartheta_{M}\right)\right|^{2} \mathrm{~d} x+\int_{\Omega} \mathrm{K}\left(\mathcal{T}_{M}\left(\vartheta_{M}\right)\right) \mid \nabla\left(\mathcal{T}_{M}\left(\vartheta_{M}\right)\right)\right)\left.\right|^{2} \mathrm{~d} x \leq & \int_{\Omega}\left|g_{\tau}^{k}+\vartheta_{\tau}^{k-1}\right|\left|\mathcal{T}_{M}\left(\vartheta_{M}\right)\right| \mathrm{d} x+\int_{\partial \Omega} h_{\tau}^{k}\left|\mathcal{T}_{M}\left(\vartheta_{M}\right)\right| \mathrm{d} S \\
& +\int_{\Omega}\left|\ell_{\tau, M}^{k}\right|\left|\mathcal{T}_{M}\left(\vartheta_{M}\right)\right|^{2} \mathrm{~d} x+\int_{\Omega}\left|j_{\tau, M}^{k}\right|\left|\mathcal{T}_{M}\left(\vartheta_{M}\right)\right| \mathrm{d} x
\end{aligned}
$$

with the place-holders

$$
\begin{aligned}
& \ell_{\tau, M}^{k}:=-\frac{\chi_{M}-\chi_{\tau}^{k-1}}{\tau}-\rho \operatorname{div}\left(\frac{\mathbf{u}_{M}-\mathbf{u}_{\tau}^{k-1}}{\tau}\right), \\
& j_{\tau, M}^{k}:=a\left(\chi_{\tau}^{k-1}\right) \varepsilon\left(\frac{\mathbf{u}_{M}-\mathbf{u}_{\tau}^{k-1}}{\tau}\right) \mathbb{V} \varepsilon\left(\frac{\mathbf{u}_{M}-\mathbf{u}_{\tau}^{k-1}}{\tau}\right)+\left|\frac{\chi_{M}-\chi_{\tau}^{k-1}}{\tau}\right|^{2}+\frac{\tau^{1 / 2}}{2}\left|\frac{\chi_{M}-\chi_{\tau}^{k-1}}{\tau}\right|^{2} .
\end{aligned}
$$

We now deal with the second term on the left-hand side of (4.28) in the same way as in the proof of [47, Thm. 2] (see also [47, Rmk. 2.10] and [23]). In fact, combining the growth condition (2.17) on K with the Poincaré inequality (2.14), and taking into account estimate (4.26), we deduce that

$$
\begin{aligned}
\exists c, C>0 \forall M>0: & \left.\int_{\Omega} \mathrm{K}\left(\mathcal{T}_{M}\left(\vartheta_{M}\right)\right) \mid \nabla\left(\mathcal{T}_{M}\left(\vartheta_{M}\right)\right)\right)\left.\right|^{2} \mathrm{~d} x \\
& \left.\geq c \| \nabla\left(\mathcal{T}_{M}\left(\vartheta_{M}\right)\right)\right)\left\|_{L^{2}\left(\Omega ; \mathbb{R}^{d}\right)}^{2}+\right\| \mathcal{T}_{M}\left(\vartheta_{M}\right) \|_{L^{3 \kappa+6}(\Omega)}^{\kappa+2}-C .
\end{aligned}
$$

Let us now consider the terms on he right-hand side of 4.28). We have

$$
\begin{aligned}
\int_{\Omega}\left|\ell_{\tau, M}^{k} \| \mathcal{T}_{M}\left(\vartheta_{M}\right)\right|^{2} \mathrm{~d} x & \leq\left\|\ell_{\tau, M}^{k}\right\|_{L^{2}(\Omega)}\left\|\mathcal{T}_{M}\left(\vartheta_{M}\right)\right\|_{L^{3}(\Omega)}\left\|\mathcal{T}_{M}\left(\vartheta_{M}\right)\right\|_{L^{6}(\Omega)} \\
& \left.\leq \frac{c}{4} \| \nabla\left(\mathcal{T}_{M}\left(\vartheta_{M}\right)\right)\right)\left\|_{L^{2}\left(\Omega ; \mathbb{R}^{d}\right)}^{2}+C\right\| \mathcal{T}_{M}\left(\vartheta_{M}\right) \|_{L^{3}(\Omega)}^{2} \\
& \left.\leq \frac{c}{2} \| \nabla\left(\mathcal{T}_{M}\left(\vartheta_{M}\right)\right)\right)\left\|_{L^{2}\left(\Omega ; \mathbb{R}^{d}\right)}^{2}+C^{\prime}\right\| \mathcal{T}_{M}\left(\vartheta_{M}\right) \|_{L^{1}(\Omega)}^{2}
\end{aligned}
$$

where we have used that $\sup _{M}\left\|\ell_{\tau, M}^{k}\right\|_{L^{2}(\Omega)} \leq C$ thanks to (4.26). The last inequality with different constant $C^{\prime}$ follows from the fact that $H^{1}(\Omega) \Subset L^{3}(\Omega) \subset L^{1}(\Omega)$, yielding that for all $\rho>0$ there exists $C_{\rho}>0$ such that $\left\|\mathcal{T}_{M}\left(\vartheta_{M}\right)\right\|_{L^{3}(\Omega)} \leq \rho\left\|\mathcal{T}_{M}\left(\vartheta_{M}\right)\right\|_{H^{1}(\Omega)}+C_{\rho}\left\|\mathcal{T}_{M}\left(\vartheta_{M}\right)\right\|_{L^{1}(\Omega)}$. In the same way, estimate (4.26) ensures that $\left\|j_{\tau, M}^{k}\right\|_{L^{2}(\Omega)} \leq C$, whence

$$
\int_{\Omega}\left|j_{\tau, M}^{k}\left\|\mathcal{T}_{M}\left(\vartheta_{M}\right) \mid \mathrm{d} x \leq C\right\| \mathcal{T}_{M}\left(\vartheta_{M}\right) \|_{L^{2}(\Omega)}\right.
$$

All in all, from (4.28), taking into account (4.26) and conditions (2.24) and (2.25) on $g$ and $h$, we deduce that

$$
\exists C>0 \quad \forall M>0: \quad\left\|\mathcal{T}_{M}\left(\vartheta_{M}\right)\right\|_{H^{1}(\Omega)}+\left\|\mathcal{T}_{M}\left(\vartheta_{M}\right)\right\|_{L^{3 \kappa+6}(\Omega)} \leq C,
$$

where the bound for $\left\|\mathcal{T}_{M}\left(\vartheta_{M}\right)\right\|_{L^{3 \kappa+6}(\Omega)}$ is due to (4.29).

Let us finally test (4.15) by $\vartheta_{M}$. We rely on the coercivity (4.13) of $\mathcal{A}_{M}^{k}$, and on the previously obtained estimates (4.26) and (4.30), and we use essentially the same arguments as for treating (4.28), estimating the terms $\ell_{\tau, M}^{k}$ and $j_{\tau, M}^{k}$ by means of (4.26). This leads to

$$
\sup _{M>0}\left(\left\|\vartheta_{M}\right\|_{H^{1}(\Omega)}+\left\|\vartheta_{M}\right\|_{L^{3 \kappa+6}\left(\mathcal{S}_{M}\right)}\right) \leq C
$$

cf. (4.29) for the bound on $\left\|\vartheta_{M}\right\|_{L^{3 \kappa+6}\left(\mathcal{S}_{M}\right)}$. 
In the end, it remains to estimate the terms $\alpha_{\nu}\left(\left(\chi_{M}-\chi_{\tau}^{k-1}\right) / \tau\right), A_{p}\left(\chi_{M}\right)$ and $\xi_{M}$ in (4.6). First of all, we may suppose that the terms $A_{p}\left(\chi_{\tau}^{k-1}\right), \xi_{\tau}^{k-1} \in \beta\left(\chi_{\tau}^{k-1}\right)$ from the previous step are bounded in $L^{2}(\Omega)$ by a constant independent of $M$. Then, we test (4.6) by $\left(A_{p}\left(\chi_{M}\right)-A_{p}\left(\chi_{\tau}^{k-1}\right)+\left(\xi_{M}-\xi_{\tau}^{k-1}\right)\right)$, thus obtaining

$$
\begin{aligned}
& \int_{\Omega} \lambda_{M}\left(A_{p}\left(\chi_{M}\right)-A_{p}\left(\chi_{\tau}^{k-1}\right)+\xi_{M}-\xi_{\tau}^{k-1}\right) \mathrm{d} x+\left\|A_{p}\left(\chi_{M}\right)+\xi_{M}\right\|_{L^{2}(\Omega)}^{2} \\
& =\int_{\Omega}\left(A_{p}\left(\chi_{M}\right)+\xi_{M}\right)\left(A_{p}\left(\chi_{\tau}^{k-1}\right)+\xi_{\tau}^{k-1}\right) \mathrm{d} x+\int_{\Omega} \mu_{M}\left(A_{p}\left(\chi_{M}\right)-A_{p}\left(\chi_{\tau}^{k-1}\right)+\xi_{M}-\xi_{\tau}^{k-1}\right) \mathrm{d} x \doteq I_{1}+I_{2} .
\end{aligned}
$$

Here, we have used the place-holders $\lambda_{M}:=\left(\chi_{M}-\chi_{\tau}^{k-1}\right) / \tau+\sqrt{\tau}\left(\chi_{M}-\chi_{\tau}^{k-1}\right) / \tau+\alpha_{\nu}\left(\left(\chi_{M}-\chi_{\tau}^{k-1}\right) / \tau\right)$ and $\mu_{M}:=\vartheta_{M}-b^{\prime}\left(\chi_{M}\right) \frac{\varepsilon\left(\mathbf{u}_{\tau}^{k-1}\right) \mathbb{E} \varepsilon\left(\mathbf{u}_{\tau}^{k-1}\right)}{2}-\gamma\left(\chi_{M}\right)-\nu\left(\chi_{M}\right)^{\eta-2} \eta$. With monotonicity arguments, we see that the first integral on the left-hand side is positive. We estimate

$$
I_{1} \leq \frac{1}{2}\left\|A_{p}\left(\chi_{M}\right)+\xi_{M}\right\|_{L^{2}(\Omega)}^{2}+\frac{1}{2}\left\|A_{p}\left(\chi_{\tau}^{k-1}\right)+\xi_{\tau}^{k-1}\right\|_{L^{2}(\Omega)}^{2} .
$$

It follows from the estimates on $\mathbf{u}_{\tau}^{k-1}, \chi_{\tau}^{k-1}$, from (4.26) for $\chi_{M}$, and from (4.31) for $\vartheta_{M}$ that $\left\|\mu_{M}\right\|_{L^{2}(\Omega)} \leq C$ for a constant independent of $M>0$. Therefore we have

$$
I_{2} \leq \frac{1}{4}\left\|A_{p}\left(\chi_{M}\right)+\xi_{M}\right\|_{L^{2}(\Omega)}^{2}+\frac{1}{4}\left\|A_{p}\left(\chi_{\tau}^{k-1}\right)+\xi_{\tau}^{k-1}\right\|_{L^{2}(\Omega)}^{2}+C .
$$

With this, we conclude that $\left\|A_{p}\left(\chi_{M}\right)+\xi_{M}\right\|_{L^{2}(\Omega)} \leq C$ for a constant independent of $M$. By the monotonicity of the operator $\beta$ (cf. [2, Lemma 3.3]), we find $\left\|A_{p}\left(\chi_{M}\right)\right\|_{L^{2}(\Omega)} \leq C$ and $\left\|\xi_{M}\right\|_{L^{2}(\Omega)} \leq C$. Then, a comparison argument in (4.6) yields

$$
\mu\left\|\alpha_{\nu}\left(\frac{\chi_{M}-\chi_{\tau}^{k-1}}{\tau}\right)\right\|_{L^{2}(\Omega)}+\left\|A_{p}\left(\chi_{M}\right)\right\|_{L^{2}(\Omega)}+\left\|\xi_{M}\right\|_{L^{2}(\Omega)} \leq C .
$$

Standard compactness arguments together with (4.31) imply that there exists $\vartheta \in H^{1}(\Omega)$ such that, up to a (not relabeled) subsequence,

$$
\vartheta_{M} \rightarrow \vartheta \text { in } H^{1}(\Omega), \quad \vartheta_{M} \rightarrow \vartheta \text { in } L^{q}(\Omega) \text { for all } q< \begin{cases}\infty & \text { if } d=2, \\ 6 & \text { if } d=3 .\end{cases}
$$

In particular, $\vartheta_{M} \rightarrow \vartheta$ in measure. Combine this with (4.27) we infer that $\mathcal{T}_{M}\left(\vartheta_{M}\right) \rightarrow \vartheta$ in measure. Therefore, in view of estimate (4.30) and of the Egorov theorem we ultimately have that

$$
\vartheta \in L^{3 \kappa+6}(\Omega), \quad \mathcal{T}_{M}\left(\vartheta_{M}\right) \rightarrow \vartheta \text { in } H^{1}(\Omega) \cap L^{3 \kappa+6}(\Omega), \quad \mathcal{T}_{M}\left(\vartheta_{M}\right) \rightarrow \vartheta \text { in } L^{q}(\Omega) \text { for all } 1 \leq q<3 \kappa+6 .
$$

Therefore, taking into account the growth condition (2.17) for $\mathrm{K}$, we have

$$
\mathrm{K}_{M}\left(\vartheta_{M}\right)=\mathrm{K}\left(\mathcal{T}_{M}\left(\vartheta_{M}\right)\right) \rightarrow \mathrm{K}(\vartheta) \text { in } L^{q}(\Omega) \text { for all } 1 \leq q<3+\frac{6}{\kappa} .
$$

We combine this with the fact that $\nabla \vartheta_{M} \rightarrow \nabla \vartheta$ in $L^{2}\left(\Omega ; \mathbb{R}^{d}\right)$. On the one hand, we infer that for some sufficiently big $s>0, \mathcal{A}_{M}^{k}\left(\vartheta_{M}\right)$ weakly converges in the space $W^{1, s}(\Omega)^{*}$ to the operator $\widetilde{\mathcal{A}}^{k}(\vartheta)$ defined by $\left\langle\widetilde{\mathcal{A}}^{k}(\vartheta), v\right\rangle_{W^{1, s}(\Omega)}:=\int_{\Omega} \mathrm{K}(\vartheta) \nabla \vartheta \nabla v \mathrm{~d} x-\int_{\partial \Omega} h_{\tau}^{k} v \mathrm{~d} x$ for all $v \in W^{1, s}(\Omega)$. On the other hand, a comparison in (4.15) shows that $\left(\mathcal{A}_{M}^{k}\left(\vartheta_{M}\right)\right)_{M}$ is bounded in $H^{1}(\Omega)^{*}$. Therefore, it is not difficult to infer that the operator $\widetilde{\mathcal{A}^{k}}(\vartheta)$ extends to $H^{1}(\Omega)$ and coincides with the operator $\mathcal{A}^{k}$ from (4.3), and that

$$
\mathcal{A}_{M}^{k}\left(\vartheta_{M}\right) \rightarrow \mathcal{A}^{k}(\vartheta) \quad \text { in } H^{1}(\Omega)^{*} \quad \text { as } M \rightarrow \infty .
$$

This allows us to pass to the limit in the elliptic operator in (4.15). Let us now comment the limit passage in the other nonlinear terms featuring in (4.15)-(4.17).

From estimates (4.26) and (4.32) we also deduce that there exist $\mathbf{u}, \chi, \xi$ and, if $\mu=1, \zeta$ such that, up to a subsequence, $\mathbf{u}_{M} \rightarrow \mathbf{u}$ in $W_{0}^{1, \eta}\left(\Omega ; \mathbb{R}^{d}\right), \chi_{M} \rightarrow \chi$ in $W^{1, p}(\Omega)$ (this follows from the fact that $\left(\chi_{M}\right)_{M}$ is bounded in $W^{1+\sigma, p}(\Omega)$ for all $0<\sigma<\frac{1}{p}$ by [50, Thm. 2, Rmk. 2.5]), $\xi_{M} \rightarrow \xi$ in $L^{2}(\Omega)$, and, if $\mu=1$, $\alpha_{\nu}\left(\left(\chi_{M}-\chi_{\tau}^{k-1}\right) / \tau\right) \rightarrow \zeta$ in $L^{2}(\Omega)$. By the strong-weak closedness in the sense of graphs of $\alpha_{\nu}$ (viewed as a 
maximal monotone graph in $\left.L^{2}(\Omega) \times L^{2}(\Omega)\right)$, we infer, in the case $\mu=1$, that $\zeta=\alpha_{\nu}\left(\left(\chi_{\tau}^{k}-\chi_{\tau}^{k-1}\right) / \tau\right)$ a.e. in $\Omega$. Analogously, the strong-weak closedness property of $\beta$ yields that $\xi \in \beta(\chi)$.

Combining the above convergences with (4.34)-4.35) we conclude that the functions $\vartheta$, u, $\chi$, $\xi, \zeta$ fulfill a.e. in $\Omega$

$\frac{\chi-\chi_{\tau}^{k-1}}{\tau}+\sqrt{\tau} \frac{\chi-\chi_{\tau}^{k-1}}{\tau}+\mu \alpha_{\nu}\left(\left(\chi-\chi_{\tau}^{k-1}\right) / \tau\right)+A_{p}(\chi)+\xi+\gamma(\chi)+\nu|\chi|^{\eta-2} \chi=-b^{\prime}(\chi) \frac{\varepsilon\left(\mathbf{u}_{\tau}^{k-1}\right) \mathbb{E} \varepsilon\left(\mathbf{u}_{\tau}^{k-1}\right)}{2}+\vartheta$

as well as

$$
\begin{aligned}
& \frac{\vartheta-\vartheta_{\tau}^{k-1}}{\tau}+\frac{\chi-\chi_{\tau}^{k-1}}{\tau} \vartheta+\rho \operatorname{div}\left(\frac{\mathbf{u}-\mathbf{u}_{\tau}^{k-1}}{\tau}\right) \vartheta+\mathcal{A}^{k}(\vartheta) \\
&=g_{\tau}^{k}+a\left(\chi_{\tau}^{k-1}\right) \Lambda_{k}+\left|\frac{\chi-\chi_{\tau}^{k-1}}{\tau}\right|^{2}+\frac{\tau^{1 / 2}}{2}\left|\frac{\chi-\chi_{\tau}^{k-1}}{\tau}\right|^{2} \quad \text { in } H^{1}(\Omega)^{*}, \\
& \frac{\mathbf{u}-2 \mathbf{u}_{\tau}^{k-1}+\mathbf{u}_{\tau}^{k-2}}{\tau^{2}}+\mathcal{V}\left(a\left(\chi_{\tau}^{k-1}\right) \frac{\mathbf{u}-\mathbf{u}_{\tau}^{k-1}}{\tau}\right)+\mathcal{E}(b(\chi) \mathbf{u})+\mathcal{C}_{\rho}(\vartheta)-\nu \operatorname{div}\left(\Gamma_{k}\right)=\mathbf{f}_{\tau}^{k} \quad \text { in } W_{0}^{1, \eta}\left(\Omega ; \mathbb{R}^{d}\right)^{*},
\end{aligned}
$$

where $\Lambda_{k}$ denotes the weak limit of $\varepsilon\left(\frac{\mathbf{u}_{M}-\mathbf{u}_{\tau}^{k-1}}{\tau}\right) \mathbb{V} \varepsilon\left(\frac{\mathbf{u}_{M}-\mathbf{u}_{\tau}^{k-1}}{\tau}\right)$ in $L^{2}(\Omega)$, and $\Gamma_{k}$ stands for the weak limit of $\left|\varepsilon\left(\mathbf{u}_{M}\right)\right|^{\eta-2} \mathbb{I} \varepsilon\left(\mathbf{u}_{M}\right)$ in $L^{\eta /(\eta-1)}\left(\Omega ; \mathbb{R}^{d}\right)$. In order to identify them, it is sufficient to test (4.16) by $\mathbf{u}_{M}$ and show that

$$
\begin{aligned}
\limsup _{M \rightarrow \infty}\left\langle-\operatorname{div}\left(\left|\varepsilon\left(\mathbf{u}_{M}\right)\right|^{\eta-2} \mathbb{I} \varepsilon\left(\mathbf{u}_{M}\right)\right), \mathbf{u}_{M}\right\rangle_{W^{1, \eta}\left(\Omega ; \mathbb{R}^{d}\right)} & =\limsup _{M \rightarrow \infty} \int_{\Omega}\left|\varepsilon\left(\mathbf{u}_{M}\right)\right|^{\eta} \mathrm{d} x \\
& \leq\left\langle-\operatorname{div}\left(\Gamma_{k}\right), \mathbf{u}\right\rangle_{W^{1, \eta}\left(\Omega ; \mathbb{R}^{d}\right)},
\end{aligned}
$$

which we can do, exploiting that $\mathbf{u}$ solves (4.38). This enables us to conclude that $\Gamma_{k}=-\operatorname{div}\left(|\varepsilon(\mathbf{u})|^{\eta-2} \mathbb{I} \varepsilon(\mathbf{u})\right)$ and that $\mathbf{u}_{M} \rightarrow \mathbf{u}$ strongly in $W^{1, \eta}\left(\Omega ; \mathbb{R}^{d}\right)$. The latter convergence clearly allows us to conclude that $\Lambda_{k}=$ $\varepsilon\left(\frac{\mathbf{u}-\mathbf{u}_{\tau}^{k-1}}{\tau}\right) \mathbb{V} \varepsilon\left(\frac{\mathbf{u}-\mathbf{u}_{\tau}^{k-1}}{\tau}\right)$. All in all, the triple $(\vartheta, \mathbf{u}, \chi)$ solves the system

$$
\begin{aligned}
& \frac{\vartheta-\vartheta_{\tau}^{k-1}}{\tau}+\frac{\chi-\chi_{\tau}^{k-1}}{\tau} \vartheta+\rho \operatorname{div}\left(\frac{\mathbf{u}-\mathbf{u}_{\tau}^{k-1}}{\tau}\right) \vartheta+\mathcal{A}^{k}(\vartheta)=g_{\tau}^{k} \\
& +a\left(\chi_{\tau}^{k-1}\right) \varepsilon\left(\frac{\mathbf{u}-\mathbf{u}_{\tau}^{k-1}}{\tau}\right) \mathbb{V} \varepsilon\left(\frac{\mathbf{u}-\mathbf{u}_{\tau}^{k-1}}{\tau}\right)+\left(1+\frac{\tau^{1 / 2}}{2}\right)\left|\frac{\chi-\chi_{\tau}^{k-1}}{\tau}\right|^{2} \quad \text { in } H^{1}(\Omega)^{*}, \\
& \frac{\mathbf{u}-2 \mathbf{u}_{\tau}^{k-1}+\mathbf{u}_{\tau}^{k-2}}{\tau^{2}}+\mathcal{V}\left(a\left(\chi_{\tau}^{k-1}\right) \frac{\mathbf{u}-\mathbf{u}_{\tau}^{k-1}}{\tau}\right)+\mathcal{E}(b(\chi) \mathbf{u})+\mathcal{C}_{\rho}(\vartheta)-\nu \operatorname{div}\left(|\varepsilon(\mathbf{u})|^{\eta-2} \mathbb{I} \varepsilon(\mathbf{u})\right)=\mathbf{f}_{\tau}^{k} \\
& \text { in } W_{0}^{1, \eta}\left(\Omega ; \mathbb{R}^{d}\right)^{*} \text {, } \\
& (1+\sqrt{\tau}) \frac{\chi-\chi_{\tau}^{k-1}}{\tau}+\mu \alpha_{\nu}\left(\frac{\chi-\chi_{\tau}^{k-1}}{\tau}\right)+A_{p}(\chi)+\xi+\gamma(\chi)+\nu|\chi|^{\eta-2} \chi \ni-b^{\prime}(\chi) \frac{\varepsilon\left(\mathbf{u}_{\tau}^{k-1}\right) \mathbb{E} \varepsilon\left(\mathbf{u}_{\tau}^{k-1}\right)}{2}+\vartheta
\end{aligned}
$$

a.e. in $\Omega$,

with $\xi \in \beta(\chi)$ a.e. in $\Omega$. It follows from Step 3 and convergences (4.33) that $\vartheta$ also fulfills the strict positivity property (4.10).

Step 5: passage to the limit as $\nu \rightarrow 0$. We now pass to the limit in (4.40)-(4.42) as $\nu \rightarrow 0$. We denote by $\left(\vartheta_{\nu}, \mathbf{u}_{\nu}, \chi_{\nu}\right)$ the solutions of (4.40)-(4.42) and, as before, obtain a series of estimates independent of the parameter $\nu$.

First, we test (4.40) by 1, (4.41) by $\frac{\mathbf{u}_{\nu}-\mathbf{u}_{\tau}^{k-1}}{\tau}$, (4.42) by $\frac{\chi_{\nu}-\chi_{\tau}^{k-1}}{\tau}$, and add the resulting relations. We thus conclude that

$$
\exists C>0 \forall \nu>0: \quad\left\|\vartheta_{\nu}\right\|_{L^{1}(\Omega)}+\left\|\mathbf{u}_{\nu}\right\|_{H^{1}\left(\Omega ; \mathbb{R}^{d}\right)}+\nu^{1 / \eta}\left\|\varepsilon\left(\mathbf{u}_{\nu}\right)\right\|_{L^{\eta}\left(\Omega ; \mathbb{R}^{d}\right)}+\left\|\chi_{\nu}\right\|_{W^{1, p}(\Omega)} \leq C .
$$


Second, we test (4.40) by $\vartheta_{\nu}^{\alpha-1}$, with $\alpha \in(0,1)$. With the very same calculations as for the Second a priori estimates, cf. also the proof of Prop. 4.10 ahead, we conclude that (cf. (3.7)) that

$$
c \int_{\Omega} \mathrm{K}\left(\vartheta_{\nu}\right)\left|\nabla \vartheta_{\nu}^{\alpha / 2}\right|^{2} \mathrm{~d} x+c \int_{\Omega}\left|\varepsilon\left(\frac{\mathbf{u}_{\nu}-\mathbf{u}_{\tau}^{k-1}}{\tau}\right)\right|^{2} \vartheta_{\nu}^{\alpha-1} \mathrm{~d} x+c \int_{\Omega}\left|\frac{\chi_{\nu}-\chi_{\tau}^{k-1}}{\tau}\right|^{2} \vartheta_{\nu}^{\alpha-1} \mathrm{~d} x \leq C+C \int_{\Omega} \vartheta_{\nu}^{\alpha+1} \mathrm{~d} x
$$

whence, with the same arguments as throughout (3.8)-(3.13), we arrive at $\int_{\Omega}\left|\nabla \vartheta_{\nu}^{(\kappa+\alpha) / 2}\right|^{2} \mathrm{~d} x \leq C$ for a constant independent of $\nu$. Then, choosing $\alpha \in(1 / 2,1)$ such that $\kappa+\alpha \geq 2$, we conclude that

$$
\left\|\vartheta_{\nu}\right\|_{H^{1}(\Omega)} \leq C
$$

and, again arguing via the nonlinear Poincaré inequality (2.14), we also have that

$$
\left\|\vartheta_{\nu}^{(\kappa+\alpha) / 2}\right\|_{H^{1}(\Omega)} \leq C .
$$

We then test (4.42) by $\left(A_{p}\left(\chi_{\nu}\right)-A_{p}\left(\chi_{\tau}^{k-1}\right)+\xi_{\nu}-\xi_{\tau}^{k-1}\right)$ and, arguing in the very same way as in Step 4 , conclude that

$$
\mu\left\|\alpha_{\nu}\left(\frac{\chi_{\nu}-\chi_{\tau}^{k-1}}{\tau}\right)\right\|_{L^{2}(\Omega)}+\left\|A_{p}\left(\chi_{\nu}\right)\right\|_{L^{2}(\Omega)}+\left\|\xi_{\nu}\right\|_{L^{2}(\Omega)} \leq C .
$$

We can now pass to the limit in system (4.40) -(4.42) as $\nu \downarrow 0$. It follows from the previously proved a priori estimates and from the same arguments as in Step 4 that, along a (not relabeled) subsequence, $\mathbf{u}_{\nu} \rightarrow \mathbf{u}$ in $H_{0}^{1}\left(\Omega ; \mathbb{R}^{d}\right), \chi_{\nu} \rightarrow \chi$ in $W^{1, p}(\Omega)$, and $\vartheta_{\nu} \rightarrow \vartheta$ in $H^{1}(\Omega)$. Using these convergences, it is not difficult to pass to the limit in (4.41) and conclude that $\mathbf{u}$ fulfills (4.5), with test functions in $W_{0}^{1, \eta}\left(\Omega ; \mathbb{R}^{d}\right)$. We then conclude (4.5) with test functions in $H_{0}^{1}\left(\Omega ; \mathbb{R}^{d}\right)$ by a density argument.

With the same argument as in Step 4 (cf. (4.39)), testing (4.41) by $\mathbf{u}_{\nu}$ we conclude that

$$
\limsup _{\nu \rightarrow 0} \int_{\Omega} \varepsilon\left(\mathbf{u}_{\nu}\right) \mathbb{E} \varepsilon\left(\mathbf{u}_{\nu}\right) \mathrm{d} x \leq \int_{\Omega} \varepsilon(\mathbf{u}) \mathbb{E} \varepsilon(\mathbf{u}) \mathrm{d} x,
$$

yielding that $\mathbf{u}_{\nu} \rightarrow \mathbf{u}$ strongly in $H^{1}\left(\Omega ; \mathbb{R}^{d}\right)$. Therefore,

$$
a\left(\chi_{\tau}^{k-1}\right) \varepsilon\left(\frac{\mathbf{u}_{\nu}-\mathbf{u}_{\tau}^{k-1}}{\tau}\right) \mathbb{V} \varepsilon\left(\frac{\mathbf{u}_{\nu}-\mathbf{u}_{\tau}^{k-1}}{\tau}\right) \rightarrow a\left(\chi_{\tau}^{k-1}\right) \varepsilon\left(\frac{\mathbf{u}-\mathbf{u}_{\tau}^{k-1}}{\tau}\right) \mathbb{V} \varepsilon\left(\frac{\mathbf{u}-\mathbf{u}_{\tau}^{k-1}}{\tau}\right) \quad \text { in } L^{1}(\Omega) .
$$

We use this information to pass to the limit in the heat equation (4.40). Moreover, estimate (4.45) allows us to conclude that, up to a subsequence, $\vartheta_{\nu}^{(\kappa+\alpha) / 2} \rightarrow \vartheta^{(\kappa+\alpha) / 2}$ in $H^{1}(\Omega)$, hence $\vartheta_{\nu}^{(\kappa+\alpha) / 2} \rightarrow \vartheta^{(\kappa+\alpha) / 2}$ in $L^{6-\epsilon}(\Omega)$ for all $\epsilon>0$, whence, taking into account the growth condition on $\mathrm{K}$, that

$$
\mathrm{K}\left(\vartheta_{\nu}\right) \rightarrow \mathrm{K}(\vartheta) \quad \text { in } L^{\gamma}(\Omega) \quad \text { with } \gamma=\frac{(6-\epsilon)(\kappa+\alpha)}{2 \kappa} \quad \text { for all } \epsilon>0 .
$$

This allows us to pass to the limit in the term $\mathrm{K}\left(\vartheta_{\nu}\right) \nabla \vartheta_{\nu}$, tested against $v \in W^{1, s}(\Omega)$ for some sufficiently big $s>0$. All in all, we infer that $(\vartheta, \mathbf{u}, \chi)$ satisfies (4.4) in some dual space $W^{1, s}(\Omega)^{*}$, such that, also, $W^{1, s}(\Omega) \subset L^{\infty}(\Omega)$ in accord with the $L^{1}$-convergence (4.47). Finally, we pass to the limit in the flow rule (4.42). Due to estimate (4.46), we have that there exist $\xi \in L^{2}(\Omega)$ and, if $\mu=1, \zeta \in L^{2}(\Omega)$ such that

$$
\alpha_{\nu}\left(\frac{\chi_{\nu}-\chi_{\tau}^{k-1}}{\tau}\right) \rightarrow \zeta, \quad \xi_{\nu} \rightarrow \xi \quad \text { in } L^{2}(\Omega) .
$$

The strong-weak closedness of $\beta$ yields that $\xi \in \beta(\chi)$ a.e. in $\Omega$. In order to conclude that, in the case $\mu=1$, $\zeta \in \alpha\left(\left(\chi-\chi_{\tau}^{k-1}\right) / \tau\right)$ a.e. in $\Omega$, we show that

$$
\limsup _{\nu \downarrow 0} \int_{\Omega} \alpha_{\nu}\left(\frac{\chi_{\nu}-\chi_{\tau}^{k-1}}{\tau}\right)\left(\frac{\chi_{\nu}-\chi_{\tau}^{k-1}}{\tau}\right) \mathrm{d} x \leq \int_{\Omega} \zeta\left(\frac{\chi-\chi_{\tau}^{k-1}}{\tau}\right) \mathrm{d} x
$$

and invoke well-knows results from the theory of maximal monotone operators.

All in all, we infer that $(\vartheta, \mathbf{u}, \chi)$ solves system (4.4)-(4.6), where the heat equation (4.4) is to be understood in $W^{1, s}(\Omega)^{*}$. 
Step 6: $H^{2}\left(\Omega ; \mathbb{R}^{d}\right)$-regularity for $\mathbf{u}_{\tau}^{k}$ and conclusion. We follows the steps of the regularity argument in the proof of [26, Lemma 4.1] proceed by induction and suppose that $\mathbf{u}_{\tau}^{k-1} \in H_{\mathrm{Dir}}^{2}\left(\Omega ; \mathbb{R}^{d}\right)$. First of all, we rewrite the discrete momentum equation (4.5) in the following form

$$
\int_{\Omega}\left(\tau a\left(\chi_{\tau}^{k-1}\right) \mathbb{V}+\tau^{2} b\left(\chi_{\tau}^{k}\right) \mathbb{E}\right) \varepsilon\left(\mathbf{u}_{\tau}^{k}\right): \varepsilon(\zeta) \mathrm{d} x=\int_{\Omega} \mathbf{h}_{\tau}^{k} \cdot \zeta \mathrm{d} x,
$$

where $\zeta \in H_{0}^{1}\left(\Omega ; \mathbb{R}^{d}\right)$ and the right-hand side (note that $\mathbf{u}_{\tau}^{k-1} \in H_{\mathrm{Dir}}^{2}\left(\Omega ; \mathbb{R}^{d}\right)$ ) is defined as

$$
\mathbf{h}_{\tau}^{k}:=-\mathbf{u}_{\tau}^{k}-\tau a\left(\chi_{\tau}^{k-1}\right) \mathbb{V} \varepsilon\left(\mathbf{u}_{\tau}^{k-1}\right)-\tau^{2} \mathcal{C}_{\rho}\left(\vartheta_{\tau}^{k}\right)+2 \mathbf{u}_{\tau}^{k-1}-\mathbf{u}_{\tau}^{k-2}+\tau^{2} \mathbf{f}_{\tau}^{k} \in L^{2}\left(\Omega ; \mathbb{R}^{d}\right) .
$$

Condition (2.16) shows

$$
\int_{\Omega}\left(\tau a\left(\chi_{\tau}^{k-1}\right) \gamma+\tau^{2} b\left(\chi_{\tau}^{k}\right)\right) \mathbb{E} \varepsilon\left(\mathbf{u}_{\tau}^{k}\right): \varepsilon(\zeta) \mathrm{d} x=\int_{\Omega} \mathbf{h}_{\tau}^{k} \cdot \zeta \mathrm{d} x .
$$

Since the coefficient function $\tau a\left(\chi_{\tau}^{k-1}\right) \gamma+\tau^{2} b\left(\chi_{\tau}^{k}\right) \in W^{1, p}(\Omega)$ in (4.48) is scalar-valued and bounded from below by a positive constant (see (2.18) ), we get $\left(\tau a\left(\chi_{\tau}^{k-1}\right) \gamma+\tau^{2} b\left(\chi_{\tau}^{k}\right)\right)^{-1} \in W^{1, p}(\Omega)$. Testing (4.48) with $\zeta=\left(\tau a\left(\chi_{\tau}^{k-1}\right) \gamma+\tau^{2} b\left(\chi_{\tau}^{k}\right)\right)^{-1} \varphi$ where $\varphi \in H_{0}^{1}\left(\Omega ; \mathbb{R}^{d}\right)$ is another test-function yields

$$
\int_{\Omega} \mathbb{E} \varepsilon\left(\mathbf{u}_{\tau}^{k}\right): \varepsilon(\varphi) \mathrm{d} x=\int_{\Omega} \widehat{\mathbf{h}}_{\tau}^{k} \cdot \varphi \mathrm{d} x
$$

with the new right-hand side

$$
\widehat{\mathbf{h}}_{\tau}^{k}:=\frac{1}{\tau a\left(\chi_{\tau}^{k-1}\right) \gamma+\tau^{2} b\left(\chi_{\tau}^{k}\right)} \mathbf{h}_{\tau}^{k}+\mathbb{E} \varepsilon\left(\mathbf{u}_{\tau}^{k}\right) \cdot \frac{\tau a^{\prime}\left(\chi_{\tau}^{k-1}\right) \gamma+\tau^{2} b^{\prime}\left(\chi_{\tau}^{k}\right)}{\tau a\left(\chi_{\tau}^{k-1}\right) \gamma+\tau^{2} b\left(\chi_{\tau}^{k}\right)} \nabla \chi_{\tau}^{k} .
$$

Since $\nabla \chi_{\tau}^{k} \in L^{p}\left(\Omega ; \mathbb{R}^{d}\right)$ and $\varepsilon\left(\mathbf{u}_{\tau}^{k}\right) \in L^{2}\left(\Omega ; \mathbb{R}^{d \times d}\right)$, we get $\widehat{\mathbf{h}}_{\tau}^{k} \in L^{2 p /(2+p)}\left(\Omega ; \mathbb{R}^{d}\right)$. Now, we can refer to the proof of [26, Lemma 4.1] where an iteration argument leads to $\widehat{\mathbf{h}}_{\tau}^{k} \in L^{2}\left(\Omega ; \mathbb{R}^{d}\right)$. Then, the regularity result [41, Lemma 3.2] yields $\mathbf{u}_{\tau}^{k} \in H_{\text {Dir }}^{2}\left(\Omega ; \mathbb{R}^{d}\right)$ as desired.

In the end, exploiting that $\mathbf{u}_{\tau}^{k} \in H^{2}\left(\Omega ; \mathbb{R}^{d}\right)$, a comparison argument in the heat equation allows us to conclude that $\int_{\Omega} \mathrm{K}(\vartheta) \nabla \vartheta \cdot \nabla v \mathrm{~d} x$ is well defined for all test functions $v \in H^{1}(\Omega)$, hence (4.4) is solved in $H^{1}(\Omega)^{*}$.

Remark 4.5. In the case $\mu=1$, as mentioned in Remark 4.3, the discrete $\chi$-equation could be decoupled from the discrete equations for $\vartheta$ and $\mathbf{u}$, cf. (4.9). This would lead to having the term $\frac{\chi_{\tau}^{k}-\chi_{\tau}^{k-1}}{\tau} \vartheta_{\tau}^{k-1}$. The argument for the strict positivity of $\vartheta_{\tau}^{k}$ in Step 3 in this case would not go through. Nonetheless, it would be possible to prove that $\vartheta_{\tau}^{k} \geq 0$ a.e. in $\Omega$, by testing the discrete heat equation by $-\left(\vartheta_{\tau}^{k}\right)^{-}$, and using that $\int_{\Omega} \frac{\chi_{\tau}^{k}-\chi_{\tau}^{k-1}}{\tau} \vartheta_{\tau}^{k-1}\left(-\left(\vartheta_{\tau}^{k}\right)^{-}\right) \mathrm{d} x \geq 0$ since $\chi_{\tau}^{k} \leq \chi_{\tau}^{k-1}$ a.e. in $\Omega$.

Remark 4.6. We briefly comment on the reason why we need to perform two distinct passages to the limit in the proof of Lemma 4.4. As the above proof shows, in the passage to limit as $\nu \rightarrow 0$ we loose the information that the right-hand side of the equation for $\vartheta$ is estimated in $L^{2}(\Omega)$. Hence, we need to carry out refined estimates on the $\vartheta$-equation (i.e., testing it by $\vartheta^{\alpha-1}$ ), where we fully exploit the growth of $\mathrm{K}$ to carry out the related calculations. Clearly, to do so we first have to pass to the limit with the truncation parameter.

4.2. Approximate entropy and total energy inequalities. Preliminarily, we establish the

Notation 4.7 (Interpolants and discrete integration-by-parts formula). Hereafter, for a given Banach space $B$ and a $K_{\tau}$-tuple $\left(\mathfrak{h}_{\tau}^{k}\right)_{k=0}^{K_{\tau}} \subset B$, we shall use the short-hand notation

$$
\mathrm{D}_{\tau, k}(\mathfrak{h}):=\frac{\mathfrak{h}_{\tau}^{k}-\mathfrak{h}_{\tau}^{k-1}}{\tau}, \quad \mathrm{D}_{\tau, k}^{2}(\mathfrak{h}):=\mathrm{D}_{\tau, k}\left(\mathrm{D}_{\tau, k}(\mathfrak{h})\right)=\frac{\mathfrak{h}_{\tau}^{k}-2 \mathfrak{h}_{\tau}^{k-1}+\mathfrak{h}_{\tau}^{k-2}}{\tau^{2}}
$$

We recall the well-known discrete by-part integration formula for all $\left\{\mathfrak{h}_{\tau}^{k}\right\}_{k=0}^{K_{\tau}} \subset B,\left\{v_{\tau}^{k}\right\}_{k=0}^{K_{\tau}} \subset B^{*}$

$$
\sum_{k=1}^{K_{\tau}} \tau\left\langle v_{\tau}^{k}, \mathrm{D}_{\tau, k}(\mathfrak{h})\right\rangle_{B}=\left\langle v_{\tau}^{K_{\tau}}, \mathfrak{h}_{\tau}^{K_{\tau}}\right\rangle_{B}-\left\langle v_{\tau}^{0}, \mathfrak{h}_{\tau}^{0}\right\rangle_{B}-\sum_{k=1}^{K_{\tau}} \tau\left\langle\mathrm{D}_{\tau, k}(v), \mathfrak{h}_{\tau}^{k-1}\right\rangle_{B}
$$


We introduce the left-continuous and right-continuous piecewise constant, and the piecewise linear interpolants of the values $\left\{\mathfrak{h}_{\tau}^{k}\right\}_{k=1}^{K_{\tau}}$ by

$$
\left.\begin{array}{lll}
\overline{\mathfrak{h}}_{\tau}:(0, T) \rightarrow B & \text { defined by } & \overline{\mathfrak{h}}_{\tau}(t):=\mathfrak{h}_{\tau}^{k}, \\
\mathfrak{h}_{\tau}:(0, T) \rightarrow B & \text { defined by } & \mathfrak{h}_{\tau}(t):=\mathfrak{h}_{\tau}^{k-1}, \\
\mathfrak{h}_{\tau}:(0, T) \rightarrow B & \text { defined by } & \mathfrak{h}_{\tau}(t):=\frac{t-t_{\tau}^{k-1}}{\tau} \mathfrak{h}_{\tau}^{k}+\frac{t_{\tau}^{k}-t}{\tau} \mathfrak{h}_{\tau}^{k-1}
\end{array}\right\} \quad \text { for } t \in\left(t_{\tau}^{k-1}, t_{\tau}^{k}\right]
$$

We also introduce the piecewise linear interpolant of the values $\left\{\left(\mathfrak{h}_{\tau}^{k}-\mathfrak{h}_{\tau}^{k-1}\right) / \tau\right\}_{k=1}^{K_{\tau}}$ (namely, the values taken by the -piecewise constant- function $\mathfrak{h}_{\tau}^{\prime}$ ), viz.

$$
\widehat{\mathfrak{h}}_{\tau}:(0, T) \rightarrow B \quad \widehat{\mathfrak{h}}_{\tau}(t):=\frac{\left(t-t_{\tau}^{k-1}\right)}{\tau} \frac{\mathfrak{h}_{\tau}^{k}-\mathfrak{h}_{\tau}^{k-1}}{\tau}+\frac{\left(t_{\tau}^{k}-t\right)}{\tau} \frac{\mathfrak{h}_{\tau}^{k-1}-\mathfrak{h}_{\tau}^{k-2}}{\tau} \quad \text { for } t \in\left(t_{\tau}^{k-1}, t_{\tau}^{k}\right]
$$

Note that $\widehat{\mathfrak{h}}_{\tau}^{\prime}(t)=\mathrm{D}_{\tau, k}^{2}(\mathfrak{h})$ for $t \in\left(t_{\tau}^{k-1}, t_{\tau}^{k}\right]$.

Furthermore, we denote by $\overline{\mathrm{t}}_{\tau}$ and by $\underline{\mathrm{t}}_{\tau}$ the left-continuous and right-continuous piecewise constant interpolants associated with the partition, i.e. $\overline{\mathbf{t}}_{\tau}(t):=t_{\tau}^{k}$ if $t_{\tau}^{k-1}<t \leq t_{\tau}^{k}$ and $\underline{\mathrm{t}}_{\tau}(t):=t_{\tau}^{k-1}$ if $t_{\tau}^{k-1} \leq t<t_{\tau}^{k}$. Clearly, for every $t \in[0, T]$ we have $\overline{\mathbf{t}}_{\tau}(t) \downarrow t$ and $\underline{\mathbf{t}}_{\tau}(t) \uparrow t$ as $\tau \rightarrow 0$.

In view of (2.23), (2.24), and (2.25), it is easy to check that the piecewise constant interpolants $\left(\overline{\mathbf{f}}_{\tau}\right)_{\tau},\left(\bar{g}_{\tau}\right)_{\tau}$, $\left(\bar{h}_{\tau}\right)_{\tau}$ of the values $\mathbf{f}_{\tau}^{k}, g_{\tau}^{k}, h_{\tau}^{k}$ (4.1) fulfill as $\tau \downarrow 0$

$$
\begin{aligned}
& \overline{\mathbf{f}}_{\tau} \rightarrow \mathbf{f} \text { in } L^{2}\left(0, T ; L^{2}\left(\Omega ; \mathbb{R}^{d}\right)\right), \\
& \bar{g}_{\tau} \rightarrow g \text { in } L^{1}\left(0, T ; L^{1}(\Omega)\right) \cap L^{2}\left(0, T ; H^{1}(\Omega)^{\prime}\right) . \\
& \bar{h}_{\tau} \rightarrow h \text { in } L^{1}\left(0, T ; L^{2}(\partial \Omega)\right) .
\end{aligned}
$$

We now rewrite the discrete equations (4.4)-(4.6) in terms of the interpolants $\bar{\vartheta}_{\tau}, \vartheta_{\tau}, \overline{\mathbf{u}}_{\tau}, \underline{\mathbf{u}}_{\tau}, \mathbf{u}_{\tau}, \widehat{\mathbf{u}}_{\tau}, \bar{\chi}_{\tau}$, $\underline{\chi}_{\tau}, \chi_{\tau}, \bar{\xi}_{\tau}$, and $\bar{\zeta}_{\tau}$ of the elements $\left(\vartheta_{\tau}^{k}, \mathbf{u}_{\tau}^{k}, \chi_{\tau}^{k}, \xi_{\tau}^{k}, \zeta_{\tau}^{k}\right)_{k=1}^{K_{\tau}}$. Indeed, we have for almost all $t \in(0, T)$

$$
\begin{aligned}
& \partial_{t} \vartheta_{\tau}(t)+\partial_{t} \chi_{\tau}(t) \bar{\vartheta}_{\tau}(t)+\rho \operatorname{div}\left(\partial_{t} \mathbf{u}_{\tau}(t)\right) \bar{\vartheta}_{\tau}(t)+\mathcal{A}^{\frac{\overline{\mathrm{t}}_{\tau}(t)}{\tau}}\left(\bar{\vartheta}_{\tau}(t)\right)=\bar{g}_{\tau}(t)+ \\
& +a\left(\underline{\chi}_{\tau}(t)\right) \varepsilon\left(\partial_{t} \mathbf{u}_{\tau}(t)\right) \mathbb{V} \varepsilon\left(\partial_{t} \mathbf{u}_{\tau}(t)\right)+\left(1+\frac{\tau^{1 / 2}}{2}\right)\left|\partial_{t} \chi_{\tau}(t)\right|^{2} \quad \text { in } B^{*}, \\
& \partial_{t} \widehat{\mathbf{u}}_{\tau}(t)+\mathcal{V}\left(a\left(\underline{\chi}_{\tau}(t)\right) \partial_{t} \mathbf{u}_{\tau}(t)\right)+\mathcal{E}\left(b\left(\bar{\chi}_{\tau}(t)\right) \overline{\mathbf{u}}_{\tau}(t)\right)+\mathcal{C}_{\rho}\left(\bar{\vartheta}_{\tau}\right)=\overline{\mathbf{f}}_{\tau}(t) \\
& \text { a.e. in } \Omega \text {, } \\
& (1+\sqrt{\tau}) \partial_{t} \chi_{\tau}(t)+\mu \bar{\zeta}_{\tau}(t)+A_{p} \bar{\chi}_{\tau}(t)+\bar{\xi}_{\tau}(t)+\gamma\left(\bar{\chi}_{\tau}(t)\right) \\
& =-b^{\prime}\left(\bar{\chi}_{\tau}(t)\right) \frac{\varepsilon\left(\underline{\mathbf{u}}_{\tau}(t)\right) \mathbb{E} \varepsilon\left(\underline{\mathbf{u}}_{\tau}(t)\right)}{2}+\bar{\vartheta}_{\tau}(t) \\
& \text { a.e. in } \Omega \text {, }
\end{aligned}
$$

with $\bar{\xi}_{\tau} \in \beta\left(\bar{\chi}_{\tau}\right)$ and $\bar{\zeta}_{\tau} \in \partial I_{(-\infty, 0]}\left(\partial_{t} \chi_{\tau}\right)$ a.e. in $\Omega \times(0, T)$.

Our next result states that the interpolants of suitable discrete solutions to system (4.4)-(4.6) also satisfy the approximate versions of the entropy inequality (2.39) and of the total energy inequality (2.40).

For stating the discrete entropy inequality (4.60) below, we need to introduce discrete test functions. Namely, with every test function $\varphi \in \mathrm{C}^{0}\left([0, T] ; W^{1, d+\epsilon}(\Omega)\right) \cap H^{1}\left(0, T ; L^{6 / 5}(\Omega)\right)$ we associate

$$
\text { for } k=1, \ldots, K_{\tau} \quad \varphi_{\tau}^{k}:=\varphi\left(t_{\tau}^{k}\right)
$$

and consider the piecewise constant and linear interpolants $\bar{\varphi}_{\tau}$ and $\varphi_{\tau}$ of the values $\left(\varphi_{\tau}^{k}\right)_{k=1}^{K_{\tau}}$. It can be shown that the following convergences hold as $\tau \rightarrow 0$

$$
\bar{\varphi}_{\tau} \rightarrow \varphi \quad \text { in } L^{\infty}\left(0, T ; W^{1, d+\epsilon}(\Omega)\right) \text { and } \quad \partial_{t} \varphi_{\tau} \rightarrow \partial_{t} \varphi \quad \text { in } L^{2}\left(0, T ; L^{6 / 5}(\Omega)\right) .
$$

Then, (4.60) is obtained by testing (4.4) by $\frac{\varphi_{\tau}^{k}}{\vartheta_{\tau}^{k}}$, for $k=1, \ldots, K_{\tau}$. 
As for the total energy inequality (4.61) below, let us mention that it results from our carefully designed time-discretization scheme, observing in addition that (4.6) is indeed the Euler-Lagrange equation for a suitable minimum problem, cf. (4.63) below, where the additional term

$$
\frac{\tau^{3 / 2}}{2} \int_{\Omega}\left|\frac{\chi_{\tau}^{k}-\chi_{\tau}^{k-1}}{\tau}\right|^{2} \mathrm{~d} x
$$

has the role to "compensate" for the possible non-convexity of $\int_{\Omega} \widehat{\gamma}(\chi) \mathrm{d} x$. Therefore, to get the discrete total energy inequality (4.61) we have added the term $\frac{\tau^{1 / 2}}{2}\left|\frac{\chi_{\tau}^{k}-\chi_{\tau}^{k-1}}{\tau}\right|^{2}$ to the right-hand side of (4.4). This will lead to the necessary cancellations, cf. (4.71) below.

Proposition 4.8 (Discrete entropy and total energy inequalities, $\mu \in\{0,1\}$ ). Under Hypotheses (I)-(III), for $\tau>0$ sufficiently small, the discrete solutions $\left(\vartheta_{\tau}^{k}, \mathbf{u}_{\tau}^{k}, \chi_{\tau}^{k}\right)_{k=1}^{K_{\tau}}$ to Problem 4.2 fulfill

- the discrete entropy inequality

$$
\begin{aligned}
& \int_{\overline{\mathbf{t}}_{\tau}(s)}^{\overline{\mathrm{t}}_{\tau}(t)} \int_{\Omega}\left(\log \left(\underline{\vartheta}_{\tau}(r)\right)+\underline{\chi}_{\tau}(r)\right) \partial_{t} \varphi_{\tau}(\tau) \mathrm{d} x \mathrm{~d} r+\rho \int_{\overline{\mathrm{t}}_{\tau}(s)}^{\overline{\mathrm{t}}_{\tau}(t)} \int_{\Omega} \operatorname{div}\left(\partial_{t} \mathbf{u}_{\tau}(r)\right) \bar{\varphi}_{\tau}(r) \mathrm{d} x \mathrm{~d} r \\
& -\int_{\overline{\mathrm{t}}_{\tau}(s)}^{\overline{\mathrm{t}}_{\tau}(t)} \int_{\Omega} \mathrm{K}\left(\bar{\vartheta}_{\tau}(r)\right) \nabla \log \left(\bar{\vartheta}_{\tau}(r)\right) \cdot \nabla \bar{\varphi}_{\tau}(r) \mathrm{d} x \mathrm{~d} r \\
& \leq \int_{\Omega}\left(\log \left(\bar{\vartheta}_{\tau}(t)\right)+\bar{\chi}_{\tau}(t)\right) \bar{\varphi}_{\tau}(t) \mathrm{d} x-\int_{\Omega}\left(\log \left(\bar{\vartheta}_{\tau}(s)\right)+\bar{\chi}_{\tau}(s)\right) \bar{\varphi}_{\tau}(s) \mathrm{d} x \\
& -\int_{\overline{\mathrm{t}}_{\tau}(s)}^{\overline{\mathrm{t}}_{\tau}(t)} \int_{\Omega} \mathrm{K}\left(\bar{\vartheta}_{\tau}(r)\right) \frac{\bar{\varphi}_{\tau}(r)}{\bar{\vartheta}_{\tau}(r)} \nabla \log \left(\bar{\vartheta}_{\tau}(r)\right) \cdot \nabla \bar{\vartheta}_{\tau}(r) \mathrm{d} x \mathrm{~d} r \\
& -\int_{\overline{\mathbf{t}}_{\tau}(s)}^{\overline{\mathrm{t}}_{\tau}(t)} \int_{\Omega}\left(\bar{g}_{\tau}(r)+a\left(\underline{\chi}_{\tau}(r)\right) \varepsilon\left(\partial_{t} \mathbf{u}_{\tau}(r)\right) \mathbb{V} \varepsilon\left(\partial_{t} \mathbf{u}_{\tau}(r)\right)+\left|\partial_{t} \chi_{\tau}(r)\right|^{2}+\frac{\tau^{3 / 2}}{2}\left|\partial_{t} \chi_{\tau}(r)\right|^{2}\right) \frac{\bar{\varphi}_{\tau}(r)}{\bar{\vartheta}_{\tau}(r)} \mathrm{d} x \mathrm{~d} r \\
& -\int_{\overline{\mathbf{t}}_{\tau}(s)}^{\overline{\mathrm{t}}_{\tau}(t)} \int_{\partial \Omega} \bar{h}_{\tau}(r) \frac{\bar{\varphi}_{\tau}(r)}{\bar{\vartheta}_{\tau}(r)} \mathrm{d} S \mathrm{~d} r \\
& \text { for all } 0 \leq s \leq t \leq T \text { and for all } \varphi \in \mathrm{C}^{0}\left([0, T] ; W^{1, d+\epsilon}(\Omega)\right) \cap H^{1}\left(0, T ; L^{6 / 5}(\Omega)\right) \text { with } \varphi \geq 0 \text {; } \\
& \text { - the discrete total energy inequality for all } 0 \leq s \leq t \leq T \text {, viz. } \\
& \begin{array}{r}
\mathscr{E}\left(\bar{\vartheta}_{\tau}(t), \overline{\mathbf{u}}_{\tau}(t), \partial_{t} \mathbf{u}_{\tau}(t), \bar{\chi}_{\tau}(t)\right) \leq \mathscr{E}\left(\bar{\vartheta}_{\tau}(s), \overline{\mathbf{u}}_{\tau}(s), \partial_{t} \mathbf{u}_{\tau}(s), \bar{\chi}_{\tau}(s)\right)+ \\
+\int_{\overline{\mathbf{t}}_{\tau}(s)}^{\overline{\mathbf{t}}_{\tau}(t)} \int_{\Omega}\left(\bar{g}_{\tau}+\overline{\mathbf{f}}_{\tau} \cdot \partial_{t} \mathbf{u}_{\tau}\right) \mathrm{d} x \mathrm{~d} r \\
+\int_{\overline{\mathbf{t}}_{\tau}(s)}^{\overline{\mathbf{t}}_{\tau}(t)} \int_{\partial \Omega} \bar{h}_{\tau} \mathrm{d} S \mathrm{~d} r
\end{array}
\end{aligned}
$$

with $\mathscr{E}$ from (2.41).

For the proof of the discrete entropy inequality, we will rely on a crucial inequality satisfied by any concave (differentiable) function $\psi: \operatorname{dom}(\psi) \rightarrow \mathbb{R}$, i.e.

$$
\psi(x)-\psi(y) \leq \psi^{\prime}(y)(x-y) \quad \text { for all } x, y \in \operatorname{dom}(\psi) .
$$

Proof. We split the proof in two steps.

Step 1: proof of the total energy inequality. Let us consider the minimum problem

$$
\begin{array}{r}
\min _{\chi \in W^{1, p}(\Omega)}\left\{\int _ { \Omega } \left(\frac{\tau^{3 / 2}}{2}\left|\frac{\chi-\chi_{\tau}^{k-1}}{\tau}\right|^{2}+\left(\frac{\chi_{\tau}^{k}-\chi_{\tau}^{k-1}}{\tau}\right) \chi\right.\right. \\
+\mu \widehat{\alpha}\left(\frac{\chi-\chi_{\tau}^{k-1}}{\tau}\right)+\frac{|\nabla \chi|^{p}}{p}+\widehat{\beta}(\chi) \\
\left.\left.+\widehat{\gamma}(\chi)+b(\chi) \frac{\varepsilon\left(\mathbf{u}_{\tau}^{k-1}\right) \mathbb{E} \varepsilon\left(\mathbf{u}_{\tau}^{k-1}\right)}{2}-\vartheta_{\tau}^{k} \chi\right) \mathrm{d} x\right\}
\end{array}
$$


where $\chi_{\tau}^{k}$ and $\vartheta_{\tau}^{k}$ are the discrete solutions from Lemma 4.4 and $\mathbf{u}_{\tau}^{k-1}, \chi_{\tau}^{k-1}$ are given from the previous step, and let $\lambda>0$ such that $\widehat{\gamma}^{\prime \prime} \geq-\lambda$ as in (2.21). Then, the function

$$
r \mapsto \widehat{\gamma}(r)+\lambda|r|^{2} \quad \text { is strictly convex. }
$$

Let $\bar{\tau}>0$ such that $\frac{1}{2 \tau}>\lambda$ for all $0<\tau \leq \bar{\tau}$. Adding and subtracting $\int_{\Omega} \lambda\left|\chi-\chi_{\tau}^{k-1}\right|^{2} \mathrm{~d} x$, we may rewrite the minimum problem (4.63) as

$$
\begin{aligned}
\min _{\chi \in W^{1, p}(\Omega)}\left\{\int_{\Omega}(\right. & \left(\frac{1}{2 \sqrt{\tau}}-\lambda\right)\left|\chi-\chi_{\tau}^{k-1}\right|^{2}+\left(\frac{\chi_{\tau}^{k}-\chi_{\tau}^{k-1}}{\tau}\right) \chi+\mu \widehat{\alpha}\left(\frac{\chi-\chi_{\tau}^{k-1}}{\tau}\right)+\frac{|\nabla \chi|^{p}}{p}+\widehat{\beta}(\chi)+\widehat{\gamma}(\chi) \\
& \left.\left.+\lambda|\chi|^{2}+b(\chi) \frac{\varepsilon\left(\mathbf{u}_{\tau}^{k-1}\right) \mathbb{E} \varepsilon\left(\mathbf{u}_{\tau}^{k-1}\right)}{2}-\vartheta_{\tau}^{k} \chi+\lambda\left|\chi_{\tau}^{k-1}\right|^{2}+2 \lambda \chi \chi_{\tau}^{k-1}\right) \mathrm{~d} x\right\} .
\end{aligned}
$$

Observe that the Euler-Lagrange equation for (4.65) is exactly (4.6). Using the convexity of $\widehat{\alpha}, \widehat{\beta}, b$, and the $\lambda$-convexity of $\widehat{\gamma}$ (whence (4.64)), it is not difficult to check that (4.6) has a unique solution. We may thus conclude that the minimum problem (4.65) has a unique solution, which coincides with the discrete solution $\chi_{\tau}^{k}$ from Lemma 4.4

Now, choosing $\chi_{\tau}^{k-1}$ as a competitor for $\chi_{\tau}^{k}$ in the minimum problem (4.63) yields

$$
\begin{gathered}
\tau \int_{\Omega}\left|\frac{\chi_{\tau}^{k}-\chi_{\tau}^{k-1}}{\tau}\right|^{2} \mathrm{~d} x+\int_{\Omega} \frac{\tau^{3 / 2}}{2}\left|\frac{\chi_{\tau}^{k}-\chi_{\tau}^{k-1}}{\tau}\right|^{2} \mathrm{~d} x+\mu \int_{\Omega} \widehat{\alpha}\left(\frac{\chi_{\tau}^{k}-\chi_{\tau}^{k-1}}{\tau}\right) \mathrm{d} x+\int_{\Omega} \frac{\left|\nabla \chi_{\tau}^{k}\right|^{p}}{p} \mathrm{~d} x+\int_{\Omega} \widehat{\beta}\left(\chi_{\tau}^{k}\right) \mathrm{d} x \\
+\int_{\Omega} \widehat{\gamma}\left(\chi_{\tau}^{k}\right) \mathrm{d} x+\int_{\Omega} b\left(\chi_{\tau}^{k}\right) \frac{\varepsilon\left(\mathbf{u}_{\tau}^{k-1}\right) \mathbb{E} \varepsilon\left(\mathbf{u}_{\tau}^{k-1}\right)}{2} \mathrm{~d} x-\int_{\Omega} \vartheta_{\tau}^{k} \chi_{\tau}^{k} \mathrm{~d} x \\
\leq \int_{\Omega} \frac{\left|\nabla \chi_{\tau}^{k-1}\right|^{p}}{p} \mathrm{~d} x+\int_{\Omega} \widehat{\beta}\left(\chi_{\tau}^{k-1}\right) \mathrm{d} x+\int_{\Omega} \widehat{\gamma}\left(\chi_{\tau}^{k-1}\right) \mathrm{d} x \\
+\int_{\Omega} b\left(\chi_{\tau}^{k-1}\right) \frac{\varepsilon\left(\mathbf{u}_{\tau}^{k-1}\right) \mathbb{E} \varepsilon\left(\mathbf{u}_{\tau}^{k-1}\right)}{2} \mathrm{~d} x-\int_{\Omega} \vartheta_{\tau}^{k} \chi_{\tau}^{k-1} \mathrm{~d} x .
\end{gathered}
$$

Hence, we test (4.5) by $\mathbf{u}_{\tau}^{k}-\mathbf{u}_{\tau}^{k-1}$ and observe that, for all $k=1, \ldots, K_{\tau}$,

$$
\begin{aligned}
& \tau \int_{\Omega} \mathrm{D}_{\tau, k}^{2}(\mathbf{u}) \cdot \mathrm{D}_{\tau, k}(\mathbf{u}) \mathrm{d} x \geq \frac{1}{2}\left\|\mathrm{D}_{\tau, k}(\mathbf{u})\right\|_{L^{2}(\Omega)}^{2}-\frac{1}{2}\left\|\mathrm{D}_{\tau, k-1}(\mathbf{u})\right\|_{L^{2}\left(\Omega ; \mathbb{R}^{d}\right)}^{2} \\
& \left\langle\mathcal{V}\left(a\left(\chi_{\tau}^{k-1}\right) \mathrm{D}_{\tau, k}(\mathbf{u})\right), \mathbf{u}_{\tau}^{k}-\mathbf{u}_{\tau}^{k-1}\right\rangle_{H^{1}(\Omega)}=\tau \int_{\Omega} a\left(\chi_{\tau}^{k-1}\right) \varepsilon\left(\frac{\mathbf{u}_{\tau}^{k}-\mathbf{u}_{\tau}^{k-1}}{\tau}\right) \mathbb{V} \varepsilon\left(\frac{\mathbf{u}_{\tau}^{k}-\mathbf{u}_{\tau}^{k-1}}{\tau}\right) \mathrm{d} x .
\end{aligned}
$$

Furthermore, we have

$$
\begin{aligned}
\left\langle\mathcal{E}\left(b\left(\chi_{\tau}^{k}\right) \mathbf{u}_{\tau}^{k}\right), \frac{\mathbf{u}_{\tau}^{k}-\mathbf{u}_{\tau}^{k-1}}{\tau}\right\rangle_{H^{1}\left(\Omega ; \mathbb{R}^{d}\right)} \geq & \frac{1}{2} \int_{\Omega} b\left(\chi_{\tau}^{k}\right) \varepsilon\left(\mathbf{u}_{\tau}^{k}\right) \mathbb{E} \varepsilon\left(\mathbf{u}_{\tau}^{k}\right) \mathrm{d} x-\frac{1}{2} \int_{\Omega} b\left(\chi_{\tau}^{k}\right) \varepsilon\left(\mathbf{u}_{\tau}^{k-1}\right) \mathbb{E} \varepsilon\left(\mathbf{u}_{\tau}^{k-1}\right) \mathrm{d} x \\
= & \frac{1}{2} \int_{\Omega} b\left(\chi_{\tau}^{k}\right) \varepsilon\left(\mathbf{u}_{\tau}^{k}\right) \mathbb{E} \varepsilon\left(\mathbf{u}_{\tau}^{k}\right) \mathrm{d} x-\frac{1}{2} \int_{\Omega} b\left(\chi_{\tau}^{k-1}\right) \varepsilon\left(\mathbf{u}_{\tau}^{k-1}\right) \mathbb{E} \varepsilon\left(\mathbf{u}_{\tau}^{k-1}\right) \mathrm{d} x \\
& -\frac{1}{2} \int_{\Omega}\left(b\left(\chi_{\tau}^{k}\right)-b\left(\chi_{\tau}^{k-1}\right)\right) \varepsilon\left(\mathbf{u}_{\tau}^{k-1}\right) \mathbb{E} \varepsilon\left(\mathbf{u}_{\tau}^{k-1}\right) \mathrm{d} x
\end{aligned}
$$

Finally,

$$
\tau\left\langle\mathcal{C}_{\rho}\left(\vartheta_{\tau}^{k}\right), \frac{\mathbf{u}_{\tau}^{k}-\mathbf{u}_{\tau}^{k-1}}{\tau}\right\rangle_{H^{1}\left(\Omega ; \mathbb{R}^{d}\right)}=-\rho \int_{\Omega} \vartheta_{\tau}^{k} \operatorname{div}\left(\frac{\mathbf{u}_{\tau}^{k}-\mathbf{u}_{\tau}^{k-1}}{\tau}\right) \mathrm{d} x
$$


Next, we multiply (4.4) by $\tau$ and integrate over $\Omega$. We add the resulting relation to the equation obtained testing (4.16) by $\mathbf{u}_{\tau}^{k}-\mathbf{u}_{\tau}^{k-1}$ and to (4.66). The terms

$$
\begin{array}{ll}
\tau \int_{\Omega} \mathrm{D}_{\tau, k}(\chi) \vartheta_{\tau}^{k} \mathrm{~d} x, & \rho \tau \int_{\Omega} \vartheta_{\tau}^{k} \operatorname{div}\left(\mathrm{D}_{\tau, k}(\mathbf{u})\right) \mathrm{d} x, \\
\tau \int_{\Omega} a\left(\chi_{\tau}^{k-1}\right) \varepsilon\left(\frac{\mathbf{u}_{\tau}^{k}-\mathbf{u}_{\tau}^{k-1}}{\tau}\right) \mathbb{V} \varepsilon\left(\frac{\mathbf{u}_{\tau}^{k}-\mathbf{u}_{\tau}^{k-1}}{\tau}\right) \mathrm{d} x, & \tau \int_{\Omega}\left|\frac{\chi_{\tau}^{k}-\chi_{\tau}^{k-1}}{\tau}\right|^{2} \mathrm{~d} x \\
\frac{\tau^{3 / 2}}{2} \int_{\Omega}\left|\frac{\chi_{\tau}^{k}-\chi_{\tau}^{k-1}}{\tau}\right|^{2} \mathrm{~d} x, & \frac{1}{2} \int_{\Omega}\left(b\left(\chi_{\tau}^{k}\right)-b\left(\chi_{\tau}^{k-1}\right)\right) \varepsilon\left(\mathbf{u}_{\tau}^{k-1}\right) \mathbb{E} \varepsilon\left(\mathbf{u}_{\tau}^{k-1}\right) \mathrm{d} x
\end{array}
$$

cancel out.

We sum over the index $k=m, \ldots, j$, for any couple of indexes $1 \leq m<j \leq K_{\tau}$. Taking into account (4.66) - (4.70), we ultimately obtain

$$
\begin{aligned}
& \int_{\Omega}\left(\vartheta_{\tau}^{j}+\frac{1}{2}\left|\mathrm{D}_{\tau, j}(\mathbf{u})\right|^{2}+\frac{1}{2} b\left(\chi_{\tau}^{j}\right) \varepsilon\left(\mathbf{u}_{\tau}^{j}\right) \mathbb{E} \varepsilon\left(\mathbf{u}_{\tau}^{j}\right)+\frac{\left|\nabla \chi_{\tau}^{j}\right|^{p}}{p}+\widehat{\beta}\left(\chi_{\tau}^{j}\right)+\widehat{\gamma}\left(\chi_{\tau}^{j}\right)\right) \mathrm{d} x \\
& \leq \int_{\Omega}\left(\vartheta_{\tau}^{m}+\frac{1}{2}\left|\mathrm{D}_{\tau, m}(\mathbf{u})\right|^{2}+\frac{1}{2} b\left(\chi_{\tau}^{m}\right) \varepsilon\left(\mathbf{u}_{\tau}^{m}\right) \mathbb{E} \varepsilon\left(\mathbf{u}_{\tau}^{m}\right)+\frac{\left|\nabla \chi_{\tau}^{m}\right|^{p}}{p}+\widehat{\beta}\left(\chi_{\tau}^{m}\right)+\widehat{\gamma}\left(\chi_{\tau}^{m}\right)\right) \mathrm{d} x \\
& \quad+\sum_{k=m}^{j} \tau\left(\int_{\Omega}\left(g_{\tau}^{k}+\mathbf{f}_{\tau}^{k} \cdot \mathrm{D}_{\tau, k}(\mathbf{u})\right) \mathrm{d} x+\int_{\partial \Omega} h_{\tau}^{k} \mathrm{~d} S\right),
\end{aligned}
$$

which yields (4.61).

Step 2: proof of the entropy inequality. Let us fix an arbitrary positive test function

$$
\varphi \in \mathrm{C}^{0}\left([0, T] ; W^{1, d+\epsilon}(\Omega)\right) \cap H^{1}\left(0, T ; L^{6 / 5}(\Omega)\right)
$$

with $\left(\varphi_{\tau}^{k}\right)_{k=1}^{K_{\tau}}$ defined by (4.58). We multiply (4.4) by $\frac{\varphi_{\tau}^{k}}{\vartheta_{\tau}^{k}} \in H^{1}(\Omega)$ (hence, an admissible test function for (4.4)) and integrate over $\Omega$. We obtain

$$
\begin{aligned}
& \int_{\Omega}\left(g_{\tau}^{k}+a\left(\chi_{\tau}^{k}\right) \varepsilon\left(\frac{\mathbf{u}_{\tau}^{k}-\mathbf{u}_{\tau}^{k-1}}{\tau}\right) \mathbb{V} \varepsilon\left(\frac{\mathbf{u}_{\tau}^{k}-\mathbf{u}_{\tau}^{k-1}}{\tau}\right)+\left|\frac{\chi_{\tau}^{k}-\chi_{\tau}^{k-1}}{\tau}\right|^{2}+\frac{\tau^{1 / 2}}{2}\left|\frac{\chi_{\tau}^{k}-\chi_{\tau}^{k-1}}{\tau}\right|^{2}\right) \frac{\varphi_{\tau}^{k}}{\vartheta_{\tau}^{k}} \mathrm{~d} x \\
& \quad+\int_{\partial \Omega} h_{\tau}^{k} \frac{\varphi_{\tau}^{k}}{\vartheta_{\tau}^{k}} \mathrm{~d} S \\
& =\int_{\Omega}\left(\frac{\vartheta_{\tau}^{k}-\vartheta_{\tau}^{k-1}}{\tau}+\frac{\chi_{\tau}^{k}-\chi_{\tau}^{k-1}}{\tau} \vartheta_{\tau}^{k}+\rho \operatorname{div}\left(\frac{\mathbf{u}_{\tau}^{k}-\mathbf{u}_{\tau}^{k-1}}{\tau}\right) \vartheta_{\tau}^{k}\right) \frac{\varphi_{\tau}^{k}}{\vartheta_{\tau}^{k}} \mathrm{~d} x+\int_{\Omega} \mathrm{K}\left(\vartheta_{\tau}^{k}\right) \nabla \vartheta_{\tau}^{k} \cdot \nabla\left(\frac{\varphi_{\tau}^{k}}{\vartheta_{\tau}^{k}}\right) \mathrm{d} x \\
& \leq \int_{\Omega}\left(\frac{\log \left(\vartheta_{\tau}^{k}\right)-\log \left(\vartheta_{\tau}^{k-1}\right)}{\tau}+\frac{\chi_{\tau}^{k}-\chi_{\tau}^{k-1}}{\tau}+\rho \operatorname{div}\left(\frac{\mathbf{u}_{\tau}^{k}-\mathbf{u}_{\tau}^{k-1}}{\tau}\right)\right) \varphi_{\tau}^{k} \mathrm{~d} x \\
& \quad+\int_{\Omega}\left(\frac{\mathrm{K}\left(\vartheta_{\tau}^{k}\right)}{\vartheta_{\tau}^{k}} \nabla \vartheta_{\tau}^{k} \cdot \nabla \varphi_{\tau}^{k}-\frac{\mathrm{K}\left(\vartheta_{\tau}^{k}\right)}{\left|\vartheta_{\tau}^{k}\right|^{2}}\left|\nabla \vartheta_{\tau}^{k}\right|^{2} \varphi_{\tau}^{k}\right) \mathrm{d} x
\end{aligned}
$$

where we have used that (cf. (4.62) $)$

$$
\frac{\vartheta_{\tau}^{k}-\vartheta_{\tau}^{k-1}}{\vartheta_{\tau}^{k}} \leq \log \left(\vartheta_{\tau}^{k}\right)-\log \left(\vartheta_{\tau}^{k-1}\right) \quad \text { a.e. in } \Omega .
$$

Note that this inequality is preserved by the positivity of the discrete test function $\varphi_{\tau}^{k}$. We now sum (4.73), multiplied by $\tau$, over $k=m, \ldots, j$, for any couple of indexes $1 \leq m<j \leq K_{\tau}$. We use the discrete by-partintegration formula (4.51), yielding

$$
\begin{aligned}
& \sum_{k=m}^{j} \tau \int_{\Omega} \mathrm{D}_{\tau, k}\left(\log \left(\vartheta_{\tau}^{k}\right)\right) \varphi_{\tau}^{k} \mathrm{~d} x=\int_{\Omega} \log \left(\vartheta_{\tau}^{j}\right) \varphi_{\tau}^{j} \mathrm{~d} x-\int_{\Omega} \log \left(\vartheta_{\tau}^{m}\right) \varphi_{\tau}^{m} \mathrm{~d} x-\sum_{k=m}^{j} \tau \int_{\Omega} \log \left(\vartheta_{\tau}^{k-1}\right) \mathrm{D}_{\tau, k}(\varphi) \mathrm{d} x \\
& \sum_{k=m}^{j} \tau \int_{\Omega} \mathrm{D}_{\tau, k}\left(\chi_{\tau}^{k}\right) \varphi_{\tau}^{k} \mathrm{~d} x=\int_{\Omega} \chi_{\tau}^{j} \varphi_{\tau}^{j} \mathrm{~d} x-\int_{\Omega} \chi_{\tau}^{m} \varphi_{\tau}^{m} \mathrm{~d} x-\sum_{k=m}^{j} \tau \int_{\Omega} \chi_{\tau}^{k-1} \mathrm{D}_{\tau, k}(\varphi) \mathrm{d} x .
\end{aligned}
$$


Inserting the two above inequalities in (4.73) (summed up over $k=m, \ldots, j$ ), rearranging terms, we conclude (4.60).

Remark 4.9. A close perusal of the proof of Proposition 4.8 reveals that, $b$ is only $\lambda$-convex, in place of convex, it is still possible to prove that the discrete equation for $\chi$ (4.6) admits a unique solution, and therefore conclude that $\chi_{\tau}^{k}$ is the unique minimizer for (4.63). This, provided we replace the $p$-Laplacian operator in (4.6) with its non-degenerate version, cf. Remark 2.11.

4.3. A priori estimates revisited. The following result collects all the a priori estimates for the approximate solutions constructed via time discretization. In particular, the proof renders on the discrete level the Second estimate, which has a nonlinear character and thus needs to be suitably translated within the frame of the discrete system (4.4)-(4.6).

Instead, we are not able to render the Sixth estimate. The ultimates reason for this is that this estimate is based on a comparison argument in the heat equation divided by $\vartheta$. On the time-discrete level, the analogue of the latter relation is somehow represented by the entropy inequality (4.60). Essentially, since (4.60) holds as an inequality, only, we are not able to recover from it the full information provided by the rescaled heat equation. Nonetheless, with careful calculations we will deduce from the entropy inequality (4.60) the following weaker version of estimate (3.34), namely

$$
\exists S>0 \forall \tau>0: \quad \sup _{\varphi \in W^{1, d+\epsilon}(\Omega),\|\varphi\|_{W^{1, d+\epsilon}(\Omega)} \leq 1} \operatorname{Var}\left(\left\langle\log \left(\bar{\vartheta}_{\tau}\right), \varphi\right\rangle_{W^{1, d+\epsilon}(\Omega)} ;[0, T]\right) \leq S
$$

for every $\epsilon>0$, where we have used the notation

$$
\begin{aligned}
& \operatorname{Var}\left(\left\langle\log \left(\bar{\vartheta}_{\tau}\right), \varphi\right\rangle_{W^{1, d+\epsilon}(\Omega)} ;[0, T]\right):= \\
& \sup _{0=\sigma_{0}<\sigma_{1}<\ldots<\sigma_{J}=T} \sum_{i=1}^{J}\left|\left\langle\log \left(\bar{\vartheta}_{\tau}\left(\sigma_{i}\right)\right), \varphi\right\rangle_{W^{1, d+\epsilon}(\Omega)}-\left\langle\log \left(\bar{\vartheta}_{\tau}\left(\sigma_{i-1}\right)\right), \varphi\right\rangle_{W^{1, d+\epsilon}(\Omega)}\right| .
\end{aligned}
$$

Thanks to a suitable abstract compactness result proved in the Appendix, Theorem A.5, estimate (4.74) turns out to be sufficient to develop the compactness arguments that will allow us to pass to the timecontinuous limit and thus prove Theorems 1 and 2, We postpone to Remark 4.11 some comments on how the $\operatorname{BV}\left([0, T] ; W^{1, d+\epsilon}(\Omega)^{*}\right)$-estimate for $\partial_{t} \log (\vartheta)$, on the time-continuous level, might be recovered.

Proposition 4.10. Assume Hypotheses (0)-(III) and (2.23)-(2.28). Let $\mu \in\{0,1\}$. Then, there exists a constant $S>0$ such that for all $\tau>0$ the following estimates

$$
\begin{aligned}
& \left\|\overline{\mathbf{u}}_{\tau}\right\|_{L^{\infty}\left(0, T ; H_{\mathrm{Dir}}^{2}\left(\Omega ; \mathbb{R}^{d}\right)\right)} \leq S, \\
& \left\|\mathbf{u}_{\tau}\right\|_{H^{1}\left(0, T ; H_{\mathrm{Dir}}^{2}\left(\Omega ; \mathbb{R}^{d}\right)\right) \cap W^{1, \infty}\left(0, T ; H_{0}^{1}\left(\Omega ; \mathbb{R}^{d}\right)\right)} \leq S, \\
& \left\|\widehat{\mathbf{u}}_{\tau}\right\|_{H^{1}\left(0, T ; L^{2}\left(\Omega ; \mathbb{R}^{d}\right)\right)} \leq S, \\
& \left\|\bar{\chi}_{\tau}\right\|_{L^{\infty}\left(0, T ; W^{1, p}(\Omega)\right)} \leq S \\
& \left\|\chi_{\tau}\right\|_{L^{\infty}\left(0, T ; W^{1, p}(\Omega)\right) \cap H^{1}\left(0, T ; L^{2}(\Omega)\right)} \leq S, \\
& \left\|\log \left(\bar{\vartheta}_{\tau}\right)\right\|_{L^{2}\left(0, T ; H^{1}(\Omega)\right)} \leq S, \\
& \left\|\bar{\vartheta}_{\tau}\right\|_{L^{2}\left(0, T ; H^{1}(\Omega)\right) \cap L^{\infty}\left(0, T ; L^{1}(\Omega)\right)} \leq S,
\end{aligned}
$$

hold, as well as estimate (4.74). Furthermore, under Hypothesis (V) (i.e. if $\kappa$ from (2.17) fulfills $1<\kappa<$ $5 / 3)$, we have in addition

$$
\sup _{\tau>0}\left\|\vartheta_{\tau}\right\|_{\mathrm{BV}\left([0, T] ; W^{2, d+\epsilon}(\Omega)^{*}\right)} \leq S \quad \text { for all } \epsilon>0 .
$$

Finally, if $\mu=0$ we also have

$$
\sup _{\tau>0}\left(\left\|\bar{\chi}_{\tau}\right\|_{L^{2}\left(0, T ; W^{1+\sigma, p}(\Omega)\right)}+\left\|\bar{\xi}_{\tau}\right\|_{L^{2}\left(0, T ; L^{2}(\Omega)\right)}\right) \leq S \quad \text { for all } 1 \leq \sigma<\frac{1}{p} .
$$


We now sketch the proof, showing how the formal a priori estimates in Section 3 can be translated in the framework of the time-discretization scheme; we shall only detail the argument leading to estimate (4.74).

Proof. From the discrete total energy inequality (4.61), arguing in the very same way as for the First a priori estimate, we deduce

$$
\left\|\bar{\vartheta}_{\tau}\right\|_{L^{\infty}\left(0, T ; L^{1}(\Omega)\right)}+\left\|\mathbf{u}_{\tau}\right\|_{W^{1, \infty}\left(0, T ; L^{2}\left(\Omega ; \mathbb{R}^{d}\right)\right)}+\left\|\nabla \bar{\chi}_{\tau}\right\|_{L^{\infty}\left(0, T ; L^{p}(\Omega)\right)} \leq C
$$

We also infer that $\left\|b\left(\bar{\chi}_{\tau}\right)^{1 / 2} \varepsilon\left(\overline{\mathbf{u}}_{\tau}\right)\right\|_{L^{\infty}\left(0, T ; L^{2}\left(\Omega ; \mathbb{R}^{d \times d}\right)\right)} \leq C$ which gives, via (2.18) and Korn's inequality, that

$$
\left\|\overline{\mathbf{u}}_{\tau}\right\|_{L^{\infty}\left(0, T ; H_{0}^{1}\left(\Omega ; \mathbb{R}^{d}\right)\right)} \leq C
$$

Next, along the lines of the Second a priori estimate, we test (4.4) by $F^{\prime}\left(\vartheta_{\tau}^{k}\right)=\left(\vartheta_{\tau}^{k}\right)^{\alpha-1}$, with $\alpha \in(0,1)$. Since $F(\vartheta)=\vartheta^{\alpha} / \alpha$ is concave, by (4.62) we have

$$
\left(\vartheta_{\tau}^{k}-\vartheta_{\tau}^{k-1}\right) F^{\prime}\left(\vartheta_{\tau}^{k}\right) \leq F\left(\vartheta_{\tau}^{k}\right)-F\left(\vartheta_{\tau}^{k-1}\right) \quad \text { a.e. in } \Omega
$$

therefore we obtain

$$
\begin{aligned}
& \int_{\Omega}\left(g_{\tau}^{k}+a\left(\chi_{\tau}^{k}\right) \varepsilon\left(\frac{\mathbf{u}_{\tau}^{k}-\mathbf{u}_{\tau}^{k-1}}{\tau}\right) \mathbb{V} \varepsilon\left(\frac{\mathbf{u}_{\tau}^{k}-\mathbf{u}_{\tau}^{k-1}}{\tau}\right)+\left(1+\frac{\tau^{1 / 2}}{2}\right)\left|\frac{\chi_{\tau}^{k}-\chi_{\tau}^{k-1}}{\tau}\right|^{2}\right) F^{\prime}\left(\vartheta_{\tau}^{k}\right) \mathrm{d} x+\int_{\partial \Omega} h_{\tau}^{k} F^{\prime}\left(\vartheta_{\tau}^{k}\right) \mathrm{d} S \\
& \leq \int_{\Omega}\left(\frac{F\left(\vartheta_{\tau}^{k}\right)-F\left(\vartheta_{\tau}^{k-1}\right)}{\tau}+\frac{\chi_{\tau}^{k}-\chi_{\tau}^{k-1}}{\tau} \vartheta_{\tau}^{k} F^{\prime}\left(\vartheta_{\tau}^{k}\right)+\rho \operatorname{div}\left(\frac{\mathbf{u}_{\tau}^{k}-\mathbf{u}_{\tau}^{k-1}}{\tau}\right) \vartheta_{\tau}^{k} F^{\prime}\left(\vartheta_{\tau}^{k}\right)+\mathrm{K}\left(\vartheta_{\tau}^{k}\right) \nabla \vartheta_{\tau}^{k} \nabla\left(F^{\prime}\left(\vartheta_{\tau}^{k}\right)\right)\right) \mathrm{d} x .
\end{aligned}
$$

Then, we multiply (4.78) by $\tau$. Summing over the index $k$ and recalling that $g \geq 0$ and $h \geq 0$, we obtain for all $t \in(0, T]$

$$
\begin{aligned}
& \frac{4(1-\alpha)}{\alpha^{2}} \int_{0}^{\overline{\mathrm{t}}_{\tau}(t)} \int_{\Omega} \mathrm{K}\left(\bar{\vartheta}_{\tau}\right)\left|\nabla\left(\left(\bar{\vartheta}_{\tau}\right)^{\alpha / 2}\right)\right|^{2} \mathrm{~d} x \mathrm{~d} s \\
& \quad+\int_{0}^{\overline{\mathbf{t}}_{\tau}(t)} \int_{\Omega}\left(c_{2}\left|\varepsilon\left(\partial_{t} \mathbf{u}_{\tau}\right)\right|^{2} F^{\prime}\left(\bar{\vartheta}_{\tau}\right)+\left(1+\frac{\tau^{1 / 2}}{2}\right)\left|\partial_{t} \chi_{\tau}\right|^{2} F^{\prime}\left(\bar{\vartheta}_{\tau}\right)\right) \mathrm{d} x \mathrm{~d} s \\
& \leq \int_{\Omega} F\left(\bar{\vartheta}_{\tau}(t)\right) \mathrm{d} x-\int_{\Omega} F\left(\vartheta_{0}\right) \mathrm{d} x+\int_{0}^{\overline{\mathrm{t}}_{\tau}(t)} \int_{\Omega}\left(\partial_{t} \chi_{\tau} \bar{\vartheta}_{\tau} F^{\prime}\left(\bar{\vartheta}_{\tau}\right)+\rho \operatorname{div}\left(\partial_{t} \mathbf{u}_{\tau}\right) \bar{\vartheta}_{\tau} F^{\prime}\left(\bar{\vartheta}_{\tau}\right)\right) \mathrm{d} x \mathrm{~d} s
\end{aligned}
$$

Starting from this inequality, we develop calculations completely analogous to the ones in Section 3 for the Second a priori estimate. In particular, we conclude that

$$
\int_{0}^{\overline{\mathrm{t}}_{\tau}(t)} \int_{\Omega} \mathrm{K}\left(\bar{\vartheta}_{\tau}\right)\left|\nabla\left(\left(\bar{\vartheta}_{\tau}\right)^{\alpha / 2}\right)\right|^{2} \mathrm{~d} x \mathrm{~d} s \leq C .
$$

The same calculations as for the Third estimate allow us then to deduce from (4.79) and (4.77) estimate 4.76g). As a byproduct of these calculations, we again have for all $\alpha \in(1 / 2,1)$

$$
\left\|\left(\bar{\vartheta}_{\tau}\right)^{(\kappa-\alpha) / 2}\right\|_{L^{2}\left(0, T ; H^{1}(\Omega)\right)},\left\|\left(\bar{\vartheta}_{\tau}\right)^{(\kappa+\alpha) / 2}\right\|_{L^{2}\left(0, T ; H^{1}(\Omega)\right)} \leq C
$$

Moreover, since

$$
\bar{\vartheta}_{\tau}(t) \geq \underline{\vartheta} \quad \text { a.e. in } \Omega \quad \text { for all } t \in[0, T]
$$

(with $\underline{\vartheta}$ from (4.25)), we also have 4.76f). 
As for the Fourth estimate, we subtract from the discrete total energy inequality (4.61) the discrete heat equation (4.4) multiplied by $\tau$ and summed over the index $k$. Therefore, we obtain for all $t \in[0, T]$

$$
\begin{aligned}
& \frac{1}{2} \int_{\Omega}\left|\partial_{t} \mathbf{u}_{\tau}\left(\overline{\mathrm{t}}_{\tau}(t)\right)\right|^{2} \mathrm{~d} x+\int_{0}^{\overline{\mathrm{t}}_{\tau}(t)} \mathrm{v}\left(a\left(\underline{\chi}_{\tau}\right) \partial_{t} \mathbf{u}_{\tau}, \partial_{t} \mathbf{u}_{\tau}\right) \mathrm{d} s+\frac{1}{2} \mathrm{e}\left(b\left(\bar{\chi}_{\tau}\left(\overline{\mathrm{t}}_{\tau}(t)\right)\right) \overline{\mathbf{u}}_{\tau}\left(\overline{\mathrm{t}}_{\tau}(t)\right), \overline{\mathbf{u}}_{\tau}\left(\overline{\mathrm{t}}_{\tau}(t)\right)\right) \\
& \quad+\left(1+\frac{\tau^{1 / 2}}{2}\right) \int_{0}^{\overline{\mathbf{t}}_{\tau}(t)} \int_{\Omega}\left|\partial_{t} \chi_{\tau}\right|^{2} \mathrm{~d} x \mathrm{~d} s+\int_{\Omega} \frac{1}{p}\left|\nabla \bar{\chi}_{\tau}\left(\overline{\mathrm{t}}_{\tau}(t)\right)\right|^{p}+W\left(\bar{\chi}_{\tau}\left(\overline{\mathrm{t}}_{\tau}(t)\right)\right) \mathrm{d} x \\
& =I_{0}+\int_{0}^{\overline{\mathrm{t}}_{\tau}(t)} \int_{\Omega} \bar{\vartheta}_{\tau}\left(\rho \operatorname{div}\left(\partial_{t} \mathbf{u}_{\tau}\right)+\partial_{t} \chi_{\tau}\right) \mathrm{d} x \mathrm{~d} s+\int_{0}^{\overline{\mathrm{t}}_{\tau}(t)} \int_{\Omega} \overline{\mathbf{f}}_{\tau} \cdot \partial_{t} \mathbf{u}_{\tau} \mathrm{d} x \mathrm{~d} s
\end{aligned}
$$

where we have used the place-holder $I_{0}=\frac{1}{2} \mathrm{e}\left(b\left(\chi_{0}\right) \mathbf{u}_{0}, \mathbf{u}_{0}\right)+\int_{\Omega}\left(\frac{1}{2}\left|\mathbf{v}_{0}\right|^{2}+\frac{1}{p}\left|\nabla \chi_{0}\right|^{p}+W\left(\chi_{0}\right)\right) \mathrm{d} x$. Exploiting (2.23) and estimate (4.76g), we control the second term on the right-hand side with $\int_{0}^{t} \int_{\Omega}\left|\partial_{t} \chi_{\tau}\right|^{2} \mathrm{~d} x \mathrm{~d} s$ and the second term on the left-hand side, which bounds $\int_{0}^{\bar{t}_{\tau}(t)}\left\|\partial_{t} \mathbf{u}_{\tau}\right\|_{H^{1}\left(\Omega ; \mathbb{R}^{d}\right)}^{2} \mathrm{~d} s$ thanks to (2.6). Therefore, we conclude that $\left\|\partial_{t} \mathbf{u}_{\tau}\right\|_{L^{2}\left(0, T ; H^{1}\left(\Omega ; \mathbb{R}^{d}\right)\right)} \leq C$, as well as estimates (4.76d)-(4.76e).

The Fifth estimate is performed on the time-discretization scheme by testing (4.5) by $-\operatorname{div}\left(\mathbb{V} \varepsilon\left(\mathbf{u}_{\tau}^{k}-\mathbf{u}_{\tau}^{k-1}\right)\right)$. For all the calculations, we refer to [47, (3.61)-(3.67)]: therein, the equation for $\mathbf{u}$ was the same as our own (1.2), but the elasticity and viscosity tensors $\mathbb{E}$ and $\mathbb{V}$ were assumed to be independent of the space variable $x$. Nonetheless, the computations from [47 carry over to the present setting, cf. also the formal calculations for the Fourth a priori estimate in Sec. 33. Therefore, we conclude estimates (4.76a) and (4.76b). A comparison argument in (4.5), joint with (2.9b), yields (4.76c).

We will now render the weaker version (4.74) of the Sixth estimate in the time-discrete setting. To do so, let us fix a partition $0=\sigma_{0}<\sigma_{1}<\ldots<\sigma_{J}=T$ of the interval $[0, T]$. Preliminarily, from the discrete entropy inequality (4.60), written on the interval $\left[\sigma_{i-1}, \sigma_{i}\right]$ and for a constant-in-time test function $\varphi \in W^{1, d+\epsilon}(\Omega)$ for some $\epsilon>0$, we deduce that

$$
\begin{aligned}
& \int_{\Omega}\left(\mathfrak{h}_{i, \tau}-\mathfrak{h}_{i-1, \tau}\right) \varphi \mathrm{d} x+\Lambda_{i, \tau}(\varphi) \geq 0 \quad \text { for all } \varphi \in W_{+}^{1, d+\epsilon}(\Omega), \\
& \int_{\Omega}\left(\mathfrak{h}_{i-1, \tau}-\mathfrak{h}_{i, \tau}\right) \varphi \mathrm{d} x-\Lambda_{i, \tau}(\varphi) \geq 0 \quad \text { for all } \varphi \in W_{-}^{1, d+\epsilon}(\Omega),
\end{aligned}
$$

where we have used the place-holders

$$
\begin{aligned}
\mathfrak{h}_{i, \tau}=\log \left(\bar{\vartheta}_{\tau}\left(\sigma_{i}\right)\right)+\bar{\chi}_{\tau}\left(\sigma_{i}\right), \\
\Lambda_{i, \tau}(\varphi)=\int_{\overline{\mathfrak{t}}_{\tau}\left(\sigma_{i-1}\right)}^{\overline{\mathfrak{t}}_{\tau}\left(\sigma_{i}\right)} \int_{\Omega} \mathrm{K}\left(\bar{\vartheta}_{\tau}\right) \nabla \log \left(\bar{\vartheta}_{\tau}\right) \cdot \nabla \varphi \mathrm{d} x \mathrm{~d} r-\rho \int_{\overline{\mathfrak{t}}_{\tau}\left(\sigma_{i-1}\right)}^{\overline{\mathfrak{t}}_{\tau}\left(\sigma_{i}\right)} \int_{\Omega} \operatorname{div}\left(\partial_{t} \mathbf{u}_{\tau}\right) \varphi \mathrm{d} x \mathrm{~d} r \\
\quad-\int_{\overline{\mathbf{t}}_{\tau}\left(\sigma_{i-1}\right)}^{\overline{\mathfrak{t}}_{\tau}\left(\sigma_{i}\right)} \int_{\Omega} \mathrm{K}\left(\bar{\vartheta}_{\tau}\right) \frac{\varphi}{\bar{\vartheta}_{\tau}} \nabla\left(\log \left(\bar{\vartheta}_{\tau}\right)\right) \nabla \bar{\vartheta}_{\tau} \mathrm{d} x \mathrm{~d} r-\int_{\overline{\mathbf{t}}_{\tau}\left(\sigma_{i-1}\right)}^{\overline{\mathbf{t}}_{\tau}\left(\sigma_{i}\right)} \int_{\partial \Omega} \bar{h}_{\tau} \frac{\varphi}{\bar{\vartheta}_{\tau}} \mathrm{d} S \mathrm{~d} r \\
\quad-\int_{\overline{\mathfrak{t}}_{\tau}\left(\sigma_{i-1}\right)}^{\overline{\mathfrak{t}}_{\tau}\left(\sigma_{i}\right)} \int_{\Omega}\left(\bar{g}_{\tau}+a\left(\underline{\chi}_{\tau}\right) \varepsilon\left(\partial_{t} \mathbf{u}_{\tau}\right) \mathbb{V} \varepsilon\left(\partial_{t} \mathbf{u}_{\tau}\right)+\left(1+\frac{\tau^{1 / 2}}{2}\right)\left|\partial_{t} \chi_{\tau}\right|^{2}\right) \frac{\varphi}{\bar{\vartheta}_{\tau}} \mathrm{d} x \mathrm{~d} r .
\end{aligned}
$$

For later use, we also introduce the place-holder

$$
\mathcal{R}_{\tau}:=\rho \operatorname{div}\left(\partial_{t} \mathbf{u}_{\tau}\right)+\mathrm{K}\left(\bar{\vartheta}_{\tau}\right)\left|\nabla\left(\log \left(\bar{\vartheta}_{\tau}\right)\right)\right|^{2}+\left(\bar{g}_{\tau}+a\left(\underline{\chi}_{\tau}\right) \varepsilon\left(\partial_{t} \mathbf{u}_{\tau}\right) \mathbb{V} \varepsilon\left(\partial_{t} \mathbf{u}_{\tau}\right)+\left(1+\frac{\tau^{1 / 2}}{2}\right)\left|\partial_{t} \chi_{\tau}\right|^{2}\right) \frac{1}{\bar{\vartheta}_{\tau}},
$$

so that $\Lambda_{i, \tau}(\varphi)$ rewrites as

$$
\Lambda_{i, \tau}(\varphi)=\int_{\overline{\mathbf{t}}_{\tau}\left(\sigma_{i-1}\right)}^{\overline{\mathbf{t}}_{\tau}\left(\sigma_{i}\right)} \int_{\Omega}\left(\mathrm{K}\left(\bar{\vartheta}_{\tau}\right) \nabla \log \left(\bar{\vartheta}_{\tau}\right) \cdot \nabla \varphi-\mathcal{R}_{\tau} \varphi\right) \mathrm{d} x \mathrm{~d} r-\int_{\overline{\mathbf{t}}_{\tau}\left(\sigma_{i-1}\right)}^{\overline{\mathbf{t}}_{\tau}\left(\sigma_{i}\right)} \int_{\partial \Omega} \bar{h}_{\tau} \frac{\varphi}{\bar{\vartheta}_{\tau}} \mathrm{d} S \mathrm{~d} r .
$$


We now estimate the total variation $\operatorname{Var}\left(\left\langle\log \left(\bar{\vartheta}_{\tau}\right)+\bar{\chi}_{\tau}, \varphi\right\rangle_{W^{1, d+\epsilon}(\Omega)} ;[0, T]\right)$ cf. (4.75), for $\varphi \in W^{1, d+\epsilon}(\Omega)$ with $\|\varphi\|_{W^{1, d+\epsilon}(\Omega)} \leq 1$, by proceeding as follows. We observe that for every fixed $\varphi \in W^{1, d+\epsilon}(\Omega)$ there holds

$$
\begin{aligned}
\left|\left\langle\mathfrak{h}_{i, \tau}-\mathfrak{h}_{i-1, \tau}, \varphi\right\rangle_{W^{1, d+\epsilon}(\Omega)}\right| \leq & \left|\int_{\Omega}\left(\mathfrak{h}_{i, \tau}-\mathfrak{h}_{i-1, \tau}\right) \varphi^{+} \mathrm{d} x+\Lambda_{i, \tau}\left(\varphi^{+}\right)\right|+\left|\Lambda_{i, \tau}\left(\varphi^{+}\right)\right| \\
& +\left|\int_{\Omega}\left(\mathfrak{h}_{i-1, \tau}-\mathfrak{h}_{i, \tau}\right)\left(-\varphi^{-}\right) \mathrm{d} x-\Lambda_{i, \tau}\left(-\varphi^{-}\right)\right|+\left|\Lambda_{i, \tau}\left(\varphi^{-}\right)\right| \\
& =\int_{\Omega}\left(\mathfrak{h}_{i, \tau}-\mathfrak{h}_{i-1, \tau}\right)|\varphi| \mathrm{d} x+\Lambda_{i, \tau}(|\varphi|)+\left|\Lambda_{i, \tau}\left(\varphi^{+}\right)\right|+\left|\Lambda_{i, \tau}\left(\varphi^{-}\right)\right|,
\end{aligned}
$$

where $\varphi^{+}\left(\varphi^{-}\right.$, resp.) denotes the positive (negative) part of $\varphi$. The last equality ensues from (4.82)-(4.83), allowing us to remove the absolute values in the first and second lines of (4.85), and from the linearity of the $\operatorname{map} \varphi \mapsto \Lambda_{i, \tau}(\varphi)$, yielding $\Lambda_{i, \tau}\left(\varphi^{+}\right)-\Lambda_{i, \tau}\left(-\varphi^{-}\right)=\Lambda_{i, \tau}(|\varphi|)$. Therefore,

$$
\begin{aligned}
& \sum_{i=1}^{J}\left|\left\langle\mathfrak{h}_{i, \tau}-\mathfrak{h}_{i-1, \tau}, \varphi\right\rangle_{W^{1, d+\epsilon}(\Omega)}\right| \\
& \stackrel{(1)}{\leq} \sum_{i=1}^{J} \int_{\Omega}\left(\mathfrak{h}_{i, \tau}-\mathfrak{h}_{i-1, \tau}\right)|\varphi| \mathrm{d} x+\Lambda_{i, \tau}(|\varphi|)+\left|\Lambda_{i, \tau}\left(\varphi^{+}\right)\right|+\left|\Lambda_{i, \tau}\left(\varphi^{-}\right)\right| .
\end{aligned}
$$

Next, rewriting $\Lambda_{i}(|\varphi|)$ by means of (4.84) we find that

$$
\begin{aligned}
\Lambda_{i}(|\varphi|)= & \sum_{i=1}^{J} \int_{\overline{\mathbf{t}}_{\tau}\left(\sigma_{i-1}\right)}^{\overline{\mathbf{t}}_{\tau}\left(\sigma_{i}\right)} \int_{\Omega} \mathrm{K}\left(\bar{\vartheta}_{\tau}\right) \nabla \log \left(\bar{\vartheta}_{\tau}\right) \cdot \nabla(|\varphi|) \mathrm{d} x \mathrm{~d} r \\
& \quad-\sum_{i=1}^{J} \int_{\overline{\mathbf{t}}_{\tau}\left(\sigma_{i-1}\right)}^{\overline{\mathfrak{t}}_{\tau}\left(\sigma_{i}\right)} \int_{\partial \Omega} \bar{h}_{\tau} \frac{|\varphi|}{\bar{\vartheta}_{\tau}} \mathrm{d} S \mathrm{~d} r-\sum_{i=1}^{J} \int_{\overline{\mathbf{t}}_{\tau}\left(\sigma_{i-1}\right)}^{\overline{\mathbf{t}}_{\tau}\left(\sigma_{i}\right)} \int_{\Omega} \mathcal{R}_{\tau}|\varphi| \mathrm{d} x \mathrm{~d} r \doteq I_{1}-I_{2}-I_{3} .
\end{aligned}
$$

We observe that (due to Hypothesis (I))

$$
\begin{aligned}
& \left|I_{1}\right| \leq \sum_{i=1}^{J} \sup _{\|\varphi\|_{W^{1, d+\epsilon}(\Omega)} \leq 1}\left|\int_{\overline{\mathbf{t}}_{\tau}\left(\sigma_{i-1}\right)}^{\overline{\mathbf{t}}_{\tau}\left(\sigma_{i}\right)} \int_{\Omega} \mathrm{K}\left(\bar{\vartheta}_{\tau}\right) \nabla \log \left(\bar{\vartheta}_{\tau}\right) \cdot \nabla(|\varphi|) \mathrm{d} x \mathrm{~d} r\right| \\
& \leq \sum_{i=1}^{J} \sup _{\|\varphi\|_{W^{1, d+\epsilon(\Omega)}} \leq 1}\|\varphi\|_{W^{1,3}(\Omega)} \int_{\overline{\mathbf{t}}_{\tau}\left(\sigma_{i-1}\right)}^{\overline{\mathfrak{t}}_{\tau}\left(\sigma_{i}\right)}\left\|\left(\bar{\vartheta}_{\tau}\right)^{(\kappa+\alpha-2) / 2} \nabla \bar{\vartheta}_{\tau}\right\|_{L^{2}\left(\Omega ; \mathbb{R}^{d}\right)}\left\|\left(\bar{\vartheta}_{\tau}\right)^{(\kappa-\alpha) / 2}\right\|_{L^{6}(\Omega)} \mathrm{d} s \\
& \leq C \int_{0}^{T}\left\|\left(\bar{\vartheta}_{\tau}\right)^{(\kappa+\alpha-2) / 2} \nabla \bar{\vartheta}_{\tau}\right\|_{L^{2}\left(\Omega ; \mathbb{R}^{d}\right)}\left\|\left(\bar{\vartheta}_{\tau}\right)^{(\kappa-\alpha) / 2}\right\|_{L^{6}(\Omega)} \mathrm{d} s,
\end{aligned}
$$

while we note that $-I_{2} \leq 0$ by the positivity of $h$. Moreover, taking into account the definition of $\mathcal{R}_{\tau}$, the fact that $\left|1 \bar{\vartheta}_{\tau}\right| \leq C$ a.e. in $\Omega \times(0, T)$ by (4.81), and the continuous embedding $W^{1, d+\epsilon}(\Omega) \subset L^{\infty}(\Omega)$, and arguing as for (3.31), we find

$$
\begin{aligned}
& \left|I_{3}\right| \leq \sum_{i=1}^{J} \sup _{\|\varphi\|_{W^{1, d+\epsilon}(\Omega)} \leq 1}\left|\int_{\overline{\mathfrak{t}}_{\tau}\left(\sigma_{i-1}\right)}^{\overline{\mathfrak{t}}_{\tau}\left(\sigma_{i}\right)} \int_{\Omega} \mathcal{R}_{\tau}\right| \varphi|\mathrm{d} x \mathrm{~d} r| \\
& \leq C \int_{0}^{T}\left(\left\|\partial_{t} \mathbf{u}_{\tau}\right\|_{H^{1}\left(\Omega ; \mathbb{R}^{d}\right)}+\int_{\Omega}\left|\bar{\vartheta}_{\tau}\right|^{\kappa+\alpha-2}\left|\nabla \bar{\vartheta}_{\tau}\right|^{2} \mathrm{~d} x+\int_{\Omega}\left|\nabla \bar{\vartheta}_{\tau}\right|^{2} \mathrm{~d} x\right. \\
& \left.\quad+\left\|\bar{g}_{\tau}\right\|_{L^{1}(\Omega)}+\left\|\varepsilon\left(\partial_{t} \mathbf{u}_{\tau}\right)\right\|_{L^{2}\left(\Omega ; \mathbb{R}^{d \times d}\right)}^{2}+\left\|\partial_{t} \chi_{\tau}\right\|_{L^{2}(\Omega)}^{2}\right) \mathrm{d} s .
\end{aligned}
$$


With the same calculations as throughout (4.87)-(4.89) we also estimate the terms $\left|\Lambda_{i}\left(\varphi^{+}\right)\right|$and $\left|\Lambda_{i}\left(\varphi^{-}\right)\right|$. Inserting the above estimates into (4.86), we find for every $\varphi \in W^{1, d+\epsilon}(\Omega)$ with $\|\varphi\|_{W^{1, d+\epsilon}(\Omega)} \leq 1$,

$$
\begin{aligned}
\sum_{i=1}^{J}\left|\left\langle\mathfrak{h}_{i, \tau}-\mathfrak{h}_{i-1, \tau}, \varphi\right\rangle_{W^{1, d+\epsilon}(\Omega)}\right| & \stackrel{(1)}{\leq} \int_{\Omega} \sum_{i=1}^{J}\left(\mathfrak{h}_{i, \tau}-\mathfrak{h}_{i-1, \tau}\right)|\varphi| \mathrm{d} x+\bar{C} \\
& =\int_{\Omega}\left(\log \left(\bar{\vartheta}_{\tau}(T)\right)+\bar{\chi}_{\tau}(T)-\log \left(\vartheta_{0}\right)-\chi_{0}\right)|\varphi| \mathrm{d} x+\bar{C} \stackrel{(2)}{\leq} C .
\end{aligned}
$$

Here, (1) with a positive constant $\bar{C}$, uniform with respect to $\varphi$, follows from the previously proved estimates (4.76b), (4.76d), (4.76e), (4.76g), and (4.79). Finally, (2) is due to (4.76d) and to the fact that $\left|\log \left(\bar{\vartheta}_{\tau}(t)\right)\right| \leq$ $C\left(\left|\bar{\vartheta}_{\tau}(t)\right|+\frac{1}{\left|\bar{\vartheta}_{\tau}(t)\right|}\right) \leq C\left(\left|\bar{\vartheta}_{\tau}(t)\right|+\frac{1}{\underline{\vartheta}(T)}\right)$ a.e. in $\Omega$ for all $t \in[0, T]$ thanks to (4.81). Using that $\left(\bar{\vartheta}_{\tau}\right)_{\tau}$ is bounded in $L^{\infty}\left(0, T ; L^{1}(\Omega)\right)$ by (4.77), we ultimately conclude that

$$
\exists C>0 \quad \forall \tau>0: \quad \sup _{\varphi \in W^{1, d+\epsilon}(\Omega),\|\varphi\|_{W^{1, d+\epsilon}(\Omega)} \leq 1} \operatorname{Var}\left(\left\langle\log \left(\bar{\vartheta}_{\tau}\right)+\bar{\chi}_{\tau}, \varphi\right\rangle_{W^{1, d+\epsilon}(\Omega)} ;[0, T]\right) \leq C .
$$

Therefore, (4.74) follows, taking into account estimate (4.76e), which in particular yields a bound for $\left(\bar{\chi}_{\tau}\right)_{\tau}$ in $\mathrm{BV}\left([0, T] ; L^{2}(\Omega)\right)$.

Under the additional Hypothesis (V), the same comparison argument in (4.4) as for the Seventh estimate yields (4.76h).

For the Eighth estimate, in the case $\mu=0$ we perform a comparison in (4.6). Based on (4.76a), (4.76d), (4.76e), and (4.76g) we conclude

$$
\sup _{\tau>0}\left(\left\|A_{p}\left(\bar{\chi}_{\tau}\right)\right\|_{L^{2}\left(0, T ; L^{2}(\Omega)\right)}+\left\|\bar{\xi}_{\tau}\right\|_{L^{2}\left(0, T ; L^{2}(\Omega)\right)}\right) \leq C
$$

whence (4.76i) by the aforementioned regularity results from [50].

Remark 4.11. Since we are not able to obtain an estimate in $\operatorname{BV}\left([0, T] ; W^{1, d+\epsilon}(\Omega)^{*}\right)$ for the family $\left(\log \left(\bar{\vartheta}_{\tau}\right)\right)_{\tau}$, in the time continuous limit the (albeit poor) regularity information

$$
\log (\vartheta) \in \mathrm{BV}\left([0, T] ; W^{1, d+\epsilon}(\Omega)^{*}\right)
$$

will be lost. Observe that it cannot be recovered from a comparison argument in the rescaled heat equation, since we will only obtain the entropic formulation of (1.1).

Still, the formal calculations from the Sixth estimate in Section 3 suggest that it should be possible to recover (4.90). Possibly, this could be done via a double approximation procedure, where one first passes to the limit in a suitable modified version of the time-discrete scheme (4.4)-(4.6) and obtains in the time-continuous limit a regularized version of system (1.1)-(1.3), allowing for a rigorous test of the heat equation by $\frac{1}{\vartheta}$. Thus, in the frame of this approximation of (1.1)-(1.3) it would be possible to prove the Sixth estimate, and hence to conclude (4.90) by a further limit passage.

\section{PASSAge to The Limit}

Let $\left(\bar{\vartheta}_{\tau}, \vartheta_{\tau}, \overline{\mathbf{u}}_{\tau}, \underline{\mathbf{u}}_{\tau}, \mathbf{u}_{\tau}, \widehat{\mathbf{u}}_{\tau}, \bar{\chi}_{\tau}, \underline{\chi}_{\tau}, \chi_{\tau}\right)_{\tau}$ be a family of approximate solutions, fulfilling the discrete version of (4.55) - (4.57) of system (1.1)-(1.3), the discrete entropy inequality (4.60) and the discrete total energy inequality (4.61): its existence is ensured by Proposition 4.8. We derive a preliminary compactness result, relying on the a priori estimates from Prop.4.10 and on an auxiliary compactness result, Theorem A.5 proved in the Appendix.

Lemma 5.1 (Compactness, $\mu \in\{0,1\})$. Under Hypotheses (O)-(III) and conditions (2.23) $-(2.28)$ on the data $\mathbf{f}, g, h, \vartheta_{0}, \mathbf{u}_{0}, \mathbf{v}_{0}, \chi_{0}$, for any sequence $\left(\tau_{k}\right)_{k} \subset(0,+\infty)$ with $\tau_{k} \downarrow 0$ as $k \rightarrow \infty$, there exist a (not relabeled) 
subsequence, and a triple $(\vartheta, \mathbf{u}, \chi)$ such that the following convergences hold

$$
\begin{aligned}
& \mathbf{u}_{\tau_{k}} \rightarrow^{*} \mathbf{u} \quad \text { in } H^{1}\left(0, T ; H_{\mathrm{Dir}}^{2}\left(\Omega ; \mathbb{R}^{d}\right)\right) \cap W^{1, \infty}\left(0, T ; H_{0}^{1}\left(\Omega ; \mathbb{R}^{d}\right)\right) \text {, } \\
& \overline{\mathbf{u}}_{\tau_{k}}, \underline{\mathbf{u}}_{\tau_{k}} \rightarrow \mathbf{u} \quad \text { in } L^{\infty}\left(0, T ; H^{2-\epsilon}\left(\Omega ; \mathbb{R}^{d}\right)\right) \text { for all } \epsilon \in(0,1] \text {, } \\
& \mathbf{u}_{\tau_{k}} \rightarrow \mathbf{u} \quad \text { in } \mathrm{C}^{0}\left([0, T] ; H^{2-\epsilon}\left(\Omega ; \mathbb{R}^{d}\right)\right) \text { for all } \epsilon \in(0,1] \text {, } \\
& \partial_{t} \widehat{\mathbf{u}}_{\tau_{k}} \rightarrow \mathbf{u}_{t t} \quad \text { in } L^{2}\left(0, T ; L^{2}\left(\Omega ; \mathbb{R}^{d}\right)\right), \\
& \partial_{t} \mathbf{u}_{\tau_{k}} \rightarrow \mathbf{u}_{t} \quad \text { in } L^{2}\left(0, T ; H^{1}\left(\Omega ; \mathbb{R}^{d}\right)\right) \text {, } \\
& \bar{\chi}_{\tau_{k}}, \underline{\chi}_{\tau_{k}}, \chi_{\tau_{k}} \rightarrow \chi \quad \text { in } L^{\infty}\left(0, T ; W^{1, p}(\Omega)\right) \cap H^{1}\left(0, T ; L^{2}(\Omega)\right) \text {, } \\
& \chi_{\tau_{k}} \rightarrow \chi \\
& \bar{\chi}_{\tau_{k}}, \underline{\chi}_{\tau_{k}} \rightarrow \chi \\
& \bar{\vartheta}_{\tau_{k}} \rightarrow \vartheta \\
& \log \left(\bar{\vartheta}_{\tau_{k}}\right) \rightarrow^{*} \log (\vartheta) \\
& \text { in } \mathrm{C}^{0}([0, T] ; X) \text { for all } X \text { such that } W^{1, p}(\Omega) \Subset X \subset L^{2}(\Omega) \text {, } \\
& \text { in } L^{\infty}(0, T ; X) \text { for all } X \text { such that } W^{1, p}(\Omega) \Subset X \subset L^{2}(\Omega) \text {, } \\
& \text { in } L^{2}\left(0, T ; H^{1}(\Omega)\right) \text {, } \\
& \log \left(\bar{\vartheta}_{\tau_{k}}\right) \rightarrow \log (\vartheta) \\
& \text { in } L^{2}\left(0, T ; H^{1}(\Omega)\right) \cap L^{\infty}\left(0, T ; W^{1, d+\epsilon}(\Omega)\right) \quad \text { for every } \epsilon>0 \text {, } \\
& \log \left(\bar{\vartheta}_{\tau_{k}}(t)\right) \rightarrow \log (\vartheta(t)) \\
& \text { in } L^{2}\left(0, T ; L^{s}(\Omega)\right) \text { for all } s \in[1,6) \text { if } d=3 \text {, and all } s \in[1, \infty) \text { if } d=2 \text {, } \\
& \bar{\vartheta}_{\tau_{k}} \rightarrow \vartheta \\
& \text { in } H^{1}(\Omega) \text { for almost all } t \in(0, T) \text {, }
\end{aligned}
$$

and $\vartheta$ also fulfills

(with $\underline{\vartheta}$ from (4.10) ).

$$
\vartheta \in L^{\infty}\left(0, T ; L^{1}(\Omega)\right), \quad \vartheta \geq \underline{\vartheta} \text { a.e. in } \Omega \times(0, T)
$$

Under the additional Hypothesis $(\boldsymbol{V})$, we also have $\vartheta \in \mathrm{BV}\left([0, T] ; W^{2, d+\epsilon}(\Omega)^{*}\right)$ for all $\epsilon>0$, and

$$
\begin{array}{ll}
\bar{\vartheta}_{\tau_{k}} \rightarrow \vartheta & \text { in } L^{2}(0, T ; Y) \text { for all } Y \text { such that } H^{1}(\Omega) \Subset Y \subset W^{2, d+\epsilon}(\Omega)^{*}, \\
\bar{\vartheta}_{\tau_{k}}(t) \rightarrow \vartheta(t) & \text { in } W^{2, d+\epsilon}(\Omega)^{*} \text { for all } t \in[0, T] .
\end{array}
$$

Proof. Due to due to estimates (4.76b) and (4.76c), there holds

$$
\begin{aligned}
&\left\|\mathbf{u}_{\tau}-\overline{\mathbf{u}}_{\tau}\right\|_{L^{\infty}\left(0, T ; H_{\mathrm{Dir}}^{2}\left(\Omega ; \mathbb{R}^{d}\right)\right)} \leq \tau^{1 / 2}\left\|\partial_{t} \mathbf{u}_{\tau}\right\|_{L^{2}\left(0, T ; H_{\mathrm{Dir}}^{2}\left(\Omega ; \mathbb{R}^{d}\right)\right)} \leq S \tau^{1 / 2} \\
&\left\|\widehat{\mathbf{u}}_{\tau}-\partial_{t} \mathbf{u}_{\tau}\right\|_{L^{\infty}\left(0, T ; L^{2}\left(\Omega ; \mathbb{R}^{d}\right)\right)} \leq \tau^{1 / 2}\left\|\partial_{t} \widehat{\mathbf{u}}_{\tau}\right\|_{L^{2}\left(0, T ; L^{2}\left(\Omega ; \mathbb{R}^{d}\right)\right)} \leq S \tau^{1 / 2}
\end{aligned}
$$

Taking into account estimates (4.76a), 4.76b), 4.76c), applying well-known weak and strong compactness results (for the latter, cf. e.g. [51]), also relying on (5.17) we conclude convergences (5.1)-(5.5). The same kind of arguments yields (5.6) $-(5.8)$ on account of estimates (4.76d) and (4.76e).

Concerning the convergence of the temperature variables, observe that the forthcoming Theorem A.5 applies to the family $\left(\log \left(\bar{\vartheta}_{\tau}\right)\right)_{\tau}$, with the choices $V=H^{1}(\Omega), p=2, Y=W^{1, d+\epsilon}(\Omega)$. Hence we conclude that, up to a subsequence the functions $\log \left(\bar{\vartheta}_{\tau_{k}}\right)$ weakly* converge to some $\lambda \in L^{2}\left(0, T ; H^{1}(\Omega)\right) \cap L^{\infty}\left(0, T ; W^{1, d+\epsilon}(\Omega)^{*}\right)$ for all $\epsilon>0$, and that $\log \left(\bar{\vartheta}_{\tau_{k}}(t)\right) \rightarrow \lambda(t)$ in $H^{1}(\Omega)$ for almost all $t \in(0, T)$. Therefore, up to a further subsequence we have $\log \left(\bar{\vartheta}_{\tau_{k}}(\cdot, t)\right) \rightarrow \lambda(\cdot, t)$ almost everywhere in $\Omega$. Thus,

$$
\bar{\vartheta}_{\tau_{k}} \rightarrow \vartheta:=e^{\lambda} \quad \text { for a.a. }(t, x) \in \Omega \times(0, T) .
$$

Writing $\lambda=\log (\vartheta)$, we immediately deduce (5.10) and (5.12). Convergence (5.11) follows from this argument: from (5.12) we gather that for almost all $t \in(0, T) \log \left(\bar{\vartheta}_{\tau_{k}}(t)\right) \rightarrow \log (\vartheta(t))$ in every Banach space $Z$ such that $H^{1}(\Omega) \Subset Z$, in particular in $L^{s}(\Omega)$ with $s$ as in (5.11). From the bound of $\left(\log \left(\bar{\vartheta}_{\tau_{k}}\right)\right)_{k}$ in $L^{2}\left(0, T ; H^{1}(\Omega)\right) \cap L^{\infty}\left(0, T ; W^{1, d+\epsilon}(\Omega)^{*}\right)$, combined with the interpolation inequality (cf. e.g. [51, Lemma 8])

$$
\forall \eta>0 \quad \exists C_{\eta}>0 \quad \forall \theta \in H^{1}(\Omega):\|\theta\|_{L^{s}(\Omega)} \leq \eta\|\theta\|_{H^{1}(\Omega)}+C_{\eta}\|\theta\|_{W^{1, d+\epsilon}(\Omega)^{*}},
$$

we also infer that the sequence $\left(\log \left(\bar{\vartheta}_{\tau_{k}}\right)\right)_{k}$ is uniformly integrable in $L^{2}\left(0, T ; L^{s}(\Omega)\right)$. Then, by e.g. 12 , Thm. III.3.6] the desired (5.11) ensues. 
Furthermore, from the bound 4.76g) for $\left(\bar{\vartheta}_{\tau_{k}}\right)_{k}$ we deduce by interpolation (cf. (3.15)) that the sequence $\left(\bar{\vartheta}_{\tau_{k}}\right)_{k}$ is uniformly integrable in $L^{h}(\Omega \times(0, T))$ for all $h \in[1,8 / 3)$ for $d=3$ and all $h \in[1,3)$ if $d=2$. Combining this with (5.18) we deduce convergence (5.13). By weak compactness arguments, (4.76g) gives the weak convergence (5.9). With a lower semicontinuity argument one also has that $\vartheta \in L^{\infty}\left(0, T ; L^{1}(\Omega)\right)$. Relying on (5.13) and on the approximate positivity property (4.81), we also conclude the last of (5.14).

Finally, under the additional Hypothesis $(\mathrm{V})$, we also dispose of the BV-estimate (4.76h) for $\left(\bar{\vartheta}_{\tau}\right)_{\tau}$. Combining this with 4.76g) and applying an Aubin-Lions type compactness result for BV-functions (see, for instance, [51, Cor. 4] or [48, Chap. 7, Cor. 4.9]) we conclude (5.15). The pointwise convergence (5.16) ensues from, e.g., [39, Thm. 6.1].

We are now in the position to develop the Proof of Theorem 1, by passing to the limit in the time-discrete

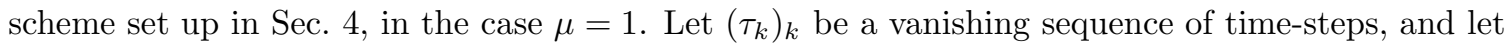

$$
\left(\bar{\vartheta}_{\tau_{k}}, \vartheta_{\tau_{k}}, \overline{\mathbf{u}}_{\tau_{k}}, \underline{\mathbf{u}}_{\tau_{k}}, \mathbf{u}_{\tau_{k}}, \widehat{\mathbf{u}}_{\tau_{k}}, \bar{\chi}_{\tau_{k}}, \underline{\chi}_{\tau_{k}}, \chi_{\tau_{k}}\right)_{k}
$$

be a sequence of approximate solutions. We can exploit the compactness results from Lemma 5.1. We split the limit passage in the following steps.

Ad the weak momentum equation (2.42). Relying on convergences (5.1), (5.4)-(5.5), on (5.8) which yields that $a\left(\underline{\chi}_{\tau_{k}}\right) \rightarrow a(\chi)$ and $b\left(\bar{\chi}_{\tau_{k}}\right) \rightarrow b(\chi)$ in $L^{p}(\Omega \times(0, T))$ for every $1 \leq p<\infty$, and on (5.9), as well as on (4.52) for $\left(\overline{\mathbf{f}}_{\tau_{k}}\right)_{k}$, we pass to the limit in the discrete momentum equation (4.56) and conclude that the triple $(\vartheta, \mathbf{u}, \chi)$ fulfills (2.42).

Ad the weak formulation (2.43) -(2.46) of the equation for $\chi, \mu=1$. The argument for obtaining (2.43)(2.46) in the limit follows exactly the same lines as the proof of [24, Thms. 4.4, 4.6] (see also [47, Thm. 3]). Therefore we only recapitulate it, referring to the latter papers for all details.

First of all, as we have pointed out in the proof of Proposition 4.8, the discrete flow rule (4.6) for $\chi$ can be interpreted as the Euler-Lagrange equation for the minimum problem (4.63), i.e. (recall that here $\mu=1$ and that $\widehat{\alpha}=I_{(-\infty, 0]}$ and $\left.\widehat{\beta}=I_{[0,+\infty)}\right)$

$$
\begin{aligned}
\min _{\chi \in W^{1, p}(\Omega)}\left\{\int _ { \Omega } \left(\frac{\tau^{3 / 2}}{2}\left|\frac{\chi-\chi_{\tau}^{k-1}}{\tau}\right|^{2}+\left(\frac{\chi_{\tau}^{k}-\chi_{\tau}^{k-1}}{\tau}\right) \chi\right.\right. & +I_{(-\infty, 0]}\left(\frac{\chi-\chi_{\tau}^{k-1}}{\tau}\right)+\frac{|\nabla \chi|^{p}}{p}+I_{[0,+\infty)}(\chi) \\
& \left.\left.+\widehat{\gamma}(\chi)+b(\chi) \frac{\varepsilon\left(\mathbf{u}_{\tau}^{k-1}\right) \mathbb{E} \varepsilon\left(\mathbf{u}_{\tau}^{k-1}\right)}{2}-\vartheta_{\tau}^{k} \chi\right) \mathrm{d} x\right\}
\end{aligned}
$$

Writing necessary optimality conditions for the minimum problem (5.19), with the very same calculations as in the proof of [47, Thm. 3], we arrive at

$$
\int_{\Omega}\left(\partial_{t} \chi_{\tau}(t) \psi+\sqrt{\tau} \partial_{t} \chi_{\tau}(t) \psi+\left|\nabla \bar{\chi}_{\tau}(t)\right|^{p-2} \nabla \bar{\chi}_{\tau}(t) \cdot \nabla \psi+\gamma\left(\bar{\chi}_{\tau}(t)\right) \psi+\bar{j}_{\tau}(t) \psi\right) \mathrm{d} x \geq 0
$$

for all $t \in[0, T]$ and all $\psi \in W^{1, p}(\Omega)$ s.t. there exists $\nu>0$ with $0 \leq \nu \psi+\bar{\chi}_{\tau}(t) \leq \underline{\chi}_{\tau}(t)$ a.e. in $\Omega$,

where where we have used the place-holder

$$
\bar{j}_{\tau}:=b^{\prime}\left(\bar{\chi}_{\tau}\right) \frac{\varepsilon\left(\underline{\mathbf{u}}_{\tau}\right) \mathbb{E} \varepsilon\left(\underline{\mathbf{u}}_{\tau}\right)}{2}-\bar{\vartheta}_{\tau} .
$$

Choosing $\psi=-\partial_{t} \chi_{\tau}(t)$ in (5.20) and summing over the index $k$ we deduce the discrete version of the energydissipation inequality (2.46) for $\chi$, holding for all $0 \leq s \leq t \leq T$, viz.

$$
\begin{aligned}
& \left.\int_{\overline{\mathbf{t}}_{\tau}(s)}^{\overline{\mathbf{t}}_{\tau}(t)} \int_{\Omega}\left(1+\tau^{1 / 2}\right)\left|\partial_{t} \chi_{\tau}\right|^{2} \mathrm{~d} x \mathrm{~d} r+\left.\int_{\Omega}\left(\frac{1}{p} \mid \nabla \bar{\chi}_{\tau}\left(\overline{\mathrm{t}}_{\tau}(t)\right)\right)\right|^{p}+W\left(\chi_{\tau}\left(\mathbf{t}_{\tau}(t)\right)\right)\right) \mathrm{d} x \\
& \leq \int_{\Omega}\left(\frac{1}{p}\left|\nabla \bar{\chi}_{\tau}\left(\overline{\mathrm{t}}_{\tau}(s)\right)\right|^{p}+W\left(\chi_{\tau}\left(\mathbf{t}_{\tau}(s)\right)\right)\right) \mathrm{d} x \\
& \quad+\int_{\overline{\mathbf{t}}_{\tau}(s)}^{\overline{\mathbf{t}}_{\tau}(t)} \int_{\Omega} \partial_{t} \chi_{\tau}\left(-b^{\prime}\left(\bar{\chi}_{\tau}\right) \frac{\varepsilon\left(\underline{\mathbf{u}}_{\tau}\right) \mathbb{E} \varepsilon\left(\underline{\mathbf{u}}_{\tau}\right)}{2}+\bar{\vartheta}_{\tau}\right) \mathrm{d} x \mathrm{~d} r+C \tau\left\|\partial_{t} \chi_{\tau}\right\|_{L^{2}\left(0, T ; L^{2}(\Omega)\right)}^{2},
\end{aligned}
$$


where we have used that

$$
\int_{\overline{\mathfrak{t}}_{\tau}(s)}^{\overline{\mathfrak{t}}_{\tau}(t)} \gamma\left(\bar{\chi}_{\tau}\right) \partial_{t} \chi_{\tau} \mathrm{d} x \mathrm{~d} r=\int_{\overline{\mathbf{t}}_{\tau}(s)}^{\overline{\mathfrak{t}}_{\tau}(t)} \gamma\left(\chi_{\tau}\right) \partial_{t} \chi_{\tau} \mathrm{d} x \mathrm{~d} r+\int_{\overline{\mathfrak{t}}_{\tau}(s)}^{\overline{\mathfrak{t}}_{\tau}(t)}\left(\gamma\left(\bar{\chi}_{\tau}\right)-\gamma\left(\chi_{\tau}\right)\right) \partial_{t} \chi_{\tau} \mathrm{d} x \mathrm{~d} r \doteq I_{1}+I_{2}
$$

and that

$$
\left.I_{1} \stackrel{(1)}{=} \int_{\Omega} \widehat{\gamma}\left(\chi_{\tau}\left(\overline{\mathrm{t}}_{\tau}(t)\right)\right) \mathrm{d} x-\int_{\Omega} \widehat{\gamma}\left(\chi_{\tau}\left(\overline{\mathrm{t}}_{\tau}(s)\right)\right) \mathrm{d} x \stackrel{(2)}{=} \int_{\Omega} W\left(\chi_{\tau}\left(\overline{\mathrm{t}}_{\tau}(t)\right)\right) \mathrm{d} x-\int_{\Omega} W\left(\chi_{\tau} \overline{\mathrm{t}}_{\tau}(s)\right)\right) \mathrm{d} x
$$

where (1) follows from the chain rule and (2) from the fact that $W=\widehat{\beta}+\widehat{\gamma}$ with $\widehat{\beta}=I_{[0,+\infty)}$. Finally,

$$
I_{2} \leq\left\|\partial_{t} \chi_{\tau}\right\|_{L^{2}\left(0, T ; L^{2}(\Omega)\right)}\left\|\gamma\left(\chi_{\tau}\right)-\gamma\left(\bar{\chi}_{\tau}\right)\right\|_{L^{2}\left(0, T ; L^{2}(\Omega)\right)} \leq C \tau\left\|\partial_{t} \chi_{\tau}\right\|_{L^{2}\left(0, T ; L^{2}(\Omega)\right)}^{2}
$$

thanks to the Lipschitz continuity of $\gamma$.

Second, repeating the "recovery sequence" argument from [24, proof of Thm. 4.4], we improve the weak convergence (5.6) to

$$
\bar{\chi}_{\tau_{k}} \rightarrow \chi \quad \text { in } L^{p}\left(0, T ; W^{1, p}(\Omega)\right) .
$$

We refer to [24] and 47] for all the related calculations.

We are now in the position to take the limit as $\tau_{k} \downarrow 0$ in the approximate energy-dissipation energy inequality (5.22). We pass to the limit on the left-hand side by lower semicontinuity, relying on convergences (5.6) -(5.7) and on the fact that $\bar{\chi}_{\tau_{k}}(t) \rightarrow \chi(t)$ in $W^{1, p}(\Omega)$ for all $t \in[0, T]$.

For the right-hand side, we exploit the strong convergence (5.23), yielding that $\bar{\chi}_{\tau_{k}}(s) \rightarrow \chi(s)$ in $W^{1, p}(\Omega)$, whence $\bar{\chi}_{\tau_{k}}(s) \rightarrow \chi(s)$ in $\mathrm{C}^{0}(\bar{\Omega})$, for almost all $s \in(0, T)$. It follows from $\widehat{\gamma} \in \mathrm{C}^{2}(\mathbb{R})$ that $\widehat{\gamma}$ has at most quadratic growth on bounded subsets of $\mathbb{R}$. We combine this with the uniform convergence of $\left(\bar{\chi}_{\tau_{k}}(s)\right)_{k}$ to conclude that $\int_{\Omega} \widehat{\gamma}\left(\bar{\chi}_{\tau_{k}}(s)\right) \mathrm{d} x \rightarrow \int_{\Omega} \widehat{\gamma}(\chi(s)) \mathrm{d} x$ for almost all $s \in(0, T)$. Since $\widehat{\beta}=I_{[0,+\infty)}$, we have $\int_{\Omega} W\left(\bar{\chi}_{\tau_{k}}(s)\right) \mathrm{d} x \rightarrow$ $\int_{\Omega} W(\chi(s)) \mathrm{d} x$ for almost all $s \in(0, T)$. Since $\left(\chi_{\tau}\right)_{\tau}$ is bounded in $H^{1}\left(0, T ; L^{2}(\Omega)\right)$, we also have

$$
\sqrt{\tau_{k}} \partial_{t} \chi_{\tau_{k}} \rightarrow 0 \text { in } L^{2}\left(0, T ; L^{2}(\Omega)\right) .
$$

Combining the weak convergence (5.6) with the strong ones (5.2), (5.8) (yielding that $b^{\prime}\left(\bar{\chi}_{\tau_{k}}\right) \rightarrow b^{\prime}(\chi)$ in $L^{p}(\Omega \times(0, T))$ for all $\left.1 \leq p<\infty\right)$, and (5.13), we also pass to the limit in the second integral term on the righthand side of (5.22). The last summand obviously tends to zero. Therefore, we conclude the energy-dissipation inequality (2.46).

Clearly, convergence (5.6) and the fact that $\partial_{t} \chi_{\tau} \leq 0$ a.e. in $\Omega \times(0, T)$ ensure that $\chi_{t} \leq 0$.e. in $\Omega \times(0, T)$, i.e. (2.43). To obtain the variational inequality (2.44), together with (2.45), we proceed exactly as in [24, 47 . The main steps are as follows: passing to the limit in (5.20) as $\tau_{k} \downarrow 0$ with suitable test functions from 24, Lemma 5.2], also relying on (5.24), we prove that for almost all $t \in(0, T)$

$$
\begin{array}{r}
\left.\left.\int_{\Omega}\left(\chi_{t}(t) \tilde{\psi}+\mid \nabla \chi(t)\right)\right|^{p-2} \nabla \chi(t) \cdot \nabla \tilde{\psi}+\gamma(\chi(t)) \tilde{\varphi}+b^{\prime}(\chi(t)) \frac{\varepsilon(\mathbf{u}(t)) \mathbb{E} \varepsilon(\mathbf{u}(t))}{2} \tilde{\psi}-\vartheta(t) \tilde{\psi}\right) \mathrm{d} x \geq 0 \\
\text { for all } \tilde{\psi} \in W_{-}^{1, p}(\Omega) \text { with }\{\tilde{\psi}=0\} \supset\{\chi(t)=0\},
\end{array}
$$

where we have used the short-hand notation $\{f=0\}$ for $\{x \in \Omega: f(x)=0\}$. From this, arguing as in the proof of [24, Thm. 4.4] we deduce that for almost all $t \in(0, T)$

$$
\begin{aligned}
& \left.\left.\int_{\Omega}\left(\chi_{t}(t) \psi+\mid \nabla \chi(t)\right)\right|^{p-2} \nabla \chi(t) \cdot \nabla \psi+\gamma(\chi(t)) \varphi+b^{\prime}(\chi(t)) \frac{\varepsilon(\mathbf{u}(t)) \mathbb{E} \varepsilon(\mathbf{u}(t))}{2} \psi-\vartheta(t) \psi\right) \mathrm{d} x \\
& \geq \int_{\left\{\chi_{(t)=0\}}\right.}\left(\gamma(\chi(t))+b^{\prime}(\chi(t)) \frac{\varepsilon(\mathbf{u}(t)) \mathbb{E} \varepsilon(\mathbf{u}(t))}{2}-\vartheta(t)\right)^{+} \psi \mathrm{d} x \text { for all } \psi \in W_{-}^{1, p}(\Omega) .
\end{aligned}
$$

Relying on (5.25), it is possible to check that the function $\xi$ from (2.49) complies with (2.44) and (2.45), cf. 24] for all details. 
Ad the entropy inequality (2.39). Let us fix a test function $\varphi \in \mathrm{C}^{0}\left([0, T] ; W^{1, d+\epsilon}(\Omega)\right) \cap H^{1}\left(0, T ; L^{6 / 5}(\Omega)\right)$ (for some $\epsilon>0$ ), for the entropy inequality (2.39). We pass to the limit as $\tau_{k} \downarrow 0$ in the discrete entropy inequality (4.60), with the discrete test functions constructed from $\varphi$ in (4.58). In order to pass to the limit in the first two integral terms on the left-hand side of (4.60), we combine convergences (5.1), (5.8), and (5.12), with the convergence (4.59) for the test functions. In order to deal with the last integral on the left-hand side, we observe that the family

$$
\left(\mathrm{K}\left(\bar{\vartheta}_{\tau}\right) \nabla \log \left(\bar{\vartheta}_{\tau}\right)\right)_{\tau} \text { is bounded in } L^{1+\delta}\left(Q ; \mathbb{R}^{d}\right) \text { for some } \delta>0 \text {. }
$$

Indeed, the growth condition (2.17) implies that

$$
\left|\mathrm{K}\left(\bar{\vartheta}_{\tau}\right) \nabla \log \left(\bar{\vartheta}_{\tau}\right)\right| \leq C\left(\left|\bar{\vartheta}_{\tau}\right|^{\kappa-1}+\frac{1}{\bar{\vartheta}_{\tau}}\right)\left|\nabla \bar{\vartheta}_{\tau}\right| \leq\left|\leq C\left(\left|\bar{\vartheta}_{\tau}\right|^{\kappa-1}+\frac{1}{\underline{\vartheta}(T)}\right)\right| \nabla \bar{\vartheta}_{\tau} \mid \quad \text { a.e. in } \Omega \times(0, T)
$$

(also due to the strict positivity (4.81)). Thus, it remains to bound the term $\left|\bar{\vartheta}_{\tau}\right|^{\kappa-1}\left|\nabla \bar{\vartheta}_{\tau}\right|$. To do so, we observe

$$
\begin{aligned}
\iint_{Q}\left(\left|\bar{\vartheta}_{\tau}\right|^{\kappa-1}\left|\nabla \bar{\vartheta}_{\tau}\right|\right)^{r} \mathrm{~d} x \mathrm{~d} t & \leq\left\|\left(\left|\bar{\vartheta}_{\tau}\right|^{(\kappa-\alpha) / 2}\right)^{r}\right\|_{L^{2 /(2-r)}(Q)}\left\|\left(\left|\bar{\vartheta}_{\tau}\right|^{(\kappa+\alpha-2) / 2} \mid \nabla \bar{\vartheta}_{\tau_{k}}\right)^{r}\right\|_{L^{2 / r}\left(Q ; \mathbb{R}^{d}\right)} \\
& \leq C\left\|\left(\left|\bar{\vartheta}_{\tau}\right|^{(\kappa-\alpha) / 2}\right)^{r}\right\|_{L^{2 /(2-r)}(Q)}
\end{aligned}
$$

for some $r>0$ (to be chosen below), where we have exploited that $\left(\left|\bar{\vartheta}_{\tau}\right|^{(\kappa+\alpha-2) / 2} \nabla \bar{\vartheta}_{\tau}\right)_{\tau}$ is bounded in $L^{2}\left(Q ; \mathbb{R}^{d}\right)$ thanks to (3.17) (cf. also (3.13)). Indeed the latter estimate yields that $\left(\left(\bar{\vartheta}_{\tau}\right)^{(\kappa+\alpha) / 2}\right)_{\tau}$ is bounded in $L^{2}(Q)$, hence that $\left(\left(\bar{\vartheta}_{\tau}\right)^{(\kappa-\alpha) / 2}\right)_{\tau}$ is bounded in $L^{2(\kappa+\alpha) /(\kappa-\alpha)}(Q)$. Therefore, it is sufficient to choose in (55.27) $r$ such that $2 r /(2-r)=2(\kappa+\alpha) /(\kappa-\alpha)$, i.e. $r=(\kappa+\alpha) / \kappa$, which is strictly bigger than 1 . Hence, up to some subsequence $\mathrm{K}\left(\bar{\vartheta}_{\tau_{k}}\right) \nabla \log \left(\bar{\vartheta}_{\tau_{k}}\right)$ weakly converges to some $\eta$ in $L^{1+\delta}\left(Q ; \mathbb{R}^{d}\right)$. In order to identify $\eta$ as $\mathrm{K}(\vartheta) \nabla \log (\vartheta)$, we use these facts. We first show that

$$
\left|\bar{\vartheta}_{\tau_{k}}\right|^{(\kappa+\alpha-2) / 2} \nabla \bar{\vartheta}_{\tau_{k}} \rightarrow|\vartheta|^{(\kappa+\alpha-2) / 2} \nabla \vartheta \quad \text { in } L^{2}\left(Q ; \mathbb{R}^{d}\right) .
$$

Indeed, on the one hand, (5.9) gives

$$
\nabla \bar{\vartheta}_{\tau_{k}} \rightarrow \nabla \vartheta \quad \text { in } L^{2}\left(0, T ; L^{2}\left(\Omega ; \mathbb{R}^{d}\right)\right) .
$$

On the other hand, the pointwise convergence $\bar{\vartheta}_{\tau_{k}} \rightarrow \vartheta$ a.e. in $\Omega \times(0, T)$ combined with the fact that $\left(\bar{\vartheta}_{\tau_{k}}\right)_{k}$ is bounded in $L^{\kappa+\alpha}(\Omega)$ yields that $\bar{\vartheta}_{\tau_{k}} \rightarrow \vartheta$ in $L^{\kappa+\alpha-\epsilon}(\Omega)$ for all $\epsilon>0$. Therefore $\left|\bar{\vartheta}_{\tau}\right|^{(\kappa+\alpha-2) / 2} \rightarrow|\vartheta|^{(\kappa+\alpha-2) / 2}$ in $L^{\eta_{\epsilon}}(\Omega)$, with $\eta_{\epsilon}:=\frac{2(\kappa+\alpha)}{\kappa+\alpha-2}-\epsilon$, for all $\epsilon>0$. We may then choose $\epsilon>0$ such that $\eta_{\epsilon}>2$ and combine this with (5.29) to conclude (5.28), taking into account that $\left(\left|\bar{\vartheta}_{\tau_{k}}\right|^{(\kappa+\alpha-2) / 2} \nabla \bar{\vartheta}_{\tau_{k}}\right)_{k}$ is bounded in $L^{2}\left(Q ; \mathbb{R}^{d}\right)$. Second, we have that

$$
\left|\bar{\vartheta}_{\tau_{k}}\right|^{(\kappa-\alpha) / 2} \rightarrow \vartheta^{(\kappa-\alpha) / 2} \text { in } L^{2(\kappa+\alpha) /(\kappa-\alpha)-\epsilon}(\Omega) \text { for all } \epsilon>0,
$$

again due to the pointwise convergence of $\bar{\vartheta}_{\tau_{k}}$ and to the fact $\left(\bar{\vartheta}_{\tau_{k}}\right)_{k}$ is bounded in $L^{\kappa+\alpha}(\Omega)$. It follows from (5.28), (5.30), the growth condition on $\mathrm{K}$, and the Lebesgue Theorem, that

$$
\mathrm{K}\left(\bar{\vartheta}_{\tau_{k}}\right) \nabla \log \left(\bar{\vartheta}_{\tau_{k}}\right) \rightarrow \mathrm{K}(\vartheta) \nabla \log (\vartheta) \quad \text { in } L^{1+\delta}\left(Q ; \mathbb{R}^{d}\right) .
$$

This and convergence (4.59) for the discrete test functions enables us to take the limit in third term on the left-hand side of (4.60). The passage to the limit in the first two integrals on the right-hand side results from convergences (5.7), (5.12) and again (4.59). For the third term, we use that

$$
\begin{aligned}
& \limsup _{k \rightarrow \infty}\left(-\int_{\overline{\mathfrak{t}}_{\tau_{k}}(s)}^{\overline{\mathfrak{t}}_{\tau_{k}}(t)} \int_{\Omega} \mathrm{K}\left(\bar{\vartheta}_{\tau_{k}}(r)\right) \frac{\bar{\varphi}_{\tau_{k}}(r)}{\bar{\vartheta}_{\tau_{k}}(r)} \nabla \log \left(\bar{\vartheta}_{\tau_{k}}(r)\right) \cdot \nabla \bar{\vartheta}_{\tau_{k}}(r) \mathrm{d} x \mathrm{~d} r\right) \\
& =-\liminf _{k \rightarrow \infty} \int_{\overline{\mathfrak{t}}_{\tau_{k}}(s)}^{\overline{\mathfrak{t}}_{\tau_{k}}(t)} \int_{\Omega} \mathrm{K}\left(\bar{\vartheta}_{\tau_{k}}(r)\right) \bar{\varphi}_{\tau_{k}}(r)\left|\nabla \log \left(\bar{\vartheta}_{\tau_{k}}(r)\right)\right|^{2} \mathrm{~d} x \mathrm{~d} r \leq-\int_{s}^{t} \int_{\Omega} \mathrm{K}(\vartheta(r)) \varphi(r)|\nabla \log (\vartheta(r))|^{2} \mathrm{~d} x \mathrm{~d} r
\end{aligned}
$$


which results from the weak convergence (5.10), combined with the pointwise convergence $\bar{\vartheta}_{\tau_{k}} \rightarrow \vartheta$ a.e. in $\Omega \times(0, T)$, (4.59) for the discrete test functions, applying the Ioffe theorem [28]. With analogous lower semicontinuity arguments we pass to the limit in the last two integrals on the right-hand side of (4.60).

Ad the total energy inequality (2.40). It follows from passing to the limit as $\tau_{k} \downarrow 0$ in the discrete total energy inequality (4.61), based on convergences (4.52)-(4.54) for $\overline{\mathbf{f}}_{\tau_{k}}, \bar{g}_{\tau_{k}}, \bar{h}_{\tau_{k}}$, and on (5.2), (5.5), (5.7), and on the pointwise convergence (5.13). Observe that convergences (5.2), (5.5), and (5.7) are sufficient to pass to the limit on the left-hand side of (4.61), by lower semicontinuity, for all $t \in[0, T]$. However, (5.13) only guarantees that $\bar{\vartheta}_{\tau_{k}}(t) \rightarrow \vartheta(t)$ in $L^{1}(\Omega)$ for almost all $t \in(0, T)$.

Enhanced regularity and improved total energy inequality under Hypothesis (V). If in addition Hyp. (V) holds, in view of Lemma $\left[5.1 \vartheta\right.$ is in $\mathrm{BV}\left([0, T] ; W^{2, d+\epsilon}(\Omega)^{*}\right)$ for every $\epsilon>0$, and the enhanced convergences (5.15) and (5.16) hold. The latter pointwise convergence allows us to pass to the limit on the left-hand side of (4.61) for all $t \in[0, T]$. This ends the proof.

We conclude this section with the Proof of Theorem 2 2 in the case $\mu=0$. Let $\left(\tau_{k}\right)_{k}$ be a vanishing sequence of time-steps, and $\left(\bar{\vartheta}_{\tau_{k}}, \vartheta_{\tau_{k}}, \overline{\mathbf{u}}_{\tau_{k}}, \underline{\mathbf{u}}_{\tau_{k}}, \mathbf{u}_{\tau_{k}}, \widehat{\mathbf{u}}_{\tau_{k}}, \bar{\chi}_{\tau_{k}}, \underline{\chi}_{\tau_{k}}, \chi_{\tau_{k}}\right)_{k}$ be a sequence of approximate solutions; let $\left(\bar{\xi}_{\tau_{k}}\right)_{k}$ be a sequence of selections in $\beta\left(\bar{\chi}_{\tau_{k}}\right)$, such that $\left(\bar{\chi}_{\tau_{k}}, \bar{\xi}_{\tau_{k}}\right)$ satisfy for all $k \in \mathbb{N}$ the approximate equation (4.57).

In the case $\mu=0$, in addition to convergences (5.1) -(5.16), estimates (4.76i) yield, up to a subsequence, the further convergences

$$
\bar{\chi}_{\tau_{k}} \rightarrow \chi \quad \text { in } L^{2}\left(0, T ; W^{1+\sigma, p}(\Omega)\right) \text { for all } 1 \leq \sigma<\frac{1}{p}, \quad \bar{\chi}_{\tau_{k}} \rightarrow \chi \quad \text { in } L^{q}\left(0, T ; W^{1, p}(\Omega)\right) \text { for all } 1 \leq q<\infty .
$$

Furthermore, there exists $\xi \in L^{2}\left(0, T ; L^{2}(\Omega)\right)$ such that

$$
\bar{\xi}_{\tau_{k}} \rightarrow \xi \text { in } L^{2}\left(0, T ; L^{2}(\Omega)\right) .
$$

The strong convergence (5.32) and the strong-weak closedness of $\beta$ (as a maximal monotone operator from $L^{2}\left(0, T ; L^{2}(\Omega)\right)$ to $\left.L^{2}\left(0, T ; L^{2}(\Omega)\right)\right)$ immediately yield that $\xi \in \beta(\chi)$ a.e. in $\Omega \times(0, T)$.

Therefore, also exploiting convergences (5.1)-(5.9) we pass to the limit in the discrete equation for $\chi$ (4.57) and immediately conclude that the quadruple $(\vartheta, \mathbf{u}, \chi, \xi)$ fulfills the pointwise formulation (2.53)-(2.54) of the internal parameter equation (1.3).

The proof of the entropy inequality, of the total energy inequality, and of the momentum equation is clearly the same as for Theorem 1.

Under the additional Hypothesis (V), as previously seen $\vartheta$ is in $\operatorname{BV}\left([0, T] ; W^{2, d+\varepsilon}(\Omega)^{*}\right)$. We prove the weak form (2.57) of the heat equation by passing to the limit as $\tau_{k} \downarrow 0$ in the approximate heat equation (4.55), tested by an arbitrary $\varphi \in \mathrm{C}^{0}\left([0, T] ; W^{2, d+\epsilon}(\Omega)\right) \cap H^{1}\left(0, T ; L^{6 / 5}(\Omega)\right)$. The passage to the limit in the first three terms on the left-hand side, and on the first two terms on the right-hand side, results from convergences (4.53), (4.54) for $\left(\bar{g}_{\tau_{k}}\right)_{k}$ and $\left(\bar{h}_{\tau_{k}}\right)_{k}$, and from (5.1)-(5.2), (5.5)-(5.9): in particular, we exploit that $\varepsilon\left(\partial_{t} \mathbf{u}_{\tau_{k}}\right) \mathbb{E} \varepsilon\left(\partial_{t} \mathbf{u}_{\tau_{k}}\right) \rightarrow \varepsilon\left(\mathbf{u}_{t}\right) \mathbb{E} \varepsilon\left(\mathbf{u}_{t}\right)$ strongly in $L^{1}(Q)$ thanks to the strong convergence (5.5).

In order to pass to the limit with the fourth term on the left-hand side of (4.55), we need to derive a finer estimate for $\left(\mathrm{K}\left(\bar{\vartheta}_{\tau_{k}}\right) \nabla \bar{\vartheta}_{\tau_{k}}\right)_{k}$. Arguing as for (3.33) we use that

$$
\left|\mathrm{K}\left(\bar{\vartheta}_{\tau_{k}}\right) \nabla \bar{\vartheta}_{\tau_{k}}\right| \leq C\left|\bar{\vartheta}_{\tau_{k}}\right|^{(\kappa-\alpha+2) / 2}\left|\bar{\vartheta}_{\tau_{k}}\right|^{(\kappa+\alpha-2) / 2}\left|\nabla \bar{\vartheta}_{\tau_{k}}\right|+C\left|\nabla \bar{\vartheta}_{\tau_{k}}\right| .
$$

Now, $\left(\bar{\vartheta}_{\tau_{k}}\right)^{(\kappa+\alpha-2) / 2} \nabla \bar{\vartheta}_{\tau_{k}}$ is bounded in $L^{2}\left(0, T ; L^{2}\left(\Omega ; \mathbb{R}^{d}\right)\right.$ ) (thanks to (4.79) ). On the other hand, $\left(\bar{\vartheta}_{\tau_{k}}\right)_{k}$ is bounded in $L^{p}(Q)$ for all $1 \leq p<8 / 3$, in the case $d=3$ (to which we confine this discussion). Therefore, choosing $\alpha \in(1 / 2,1)$ such that $\alpha>\kappa-\frac{2}{3}$ (this can be done since $\kappa<5 / 3$ by assumption), we conclude that $\left(\left(\bar{\vartheta}_{\tau_{k}}\right)^{(\kappa-\alpha+2) / 2}\right)_{k}$ is bounded in $L^{2+\delta}(Q)$ for some $\delta>0$. Ultimately, in view of (5.34) we conclude that $\left(\mathrm{K}\left(\bar{\vartheta}_{\tau_{k}}\right) \nabla \bar{\vartheta}_{\tau_{k}}\right)_{k}$ is bounded in $L^{1+\bar{\delta}}\left(0, T ; L^{1+\bar{\delta}}(\Omega)\right)$ for some $\bar{\delta}>0$, hence

$$
\exists \eta \in L^{1+\bar{\delta}}\left(0, T ; L^{1+\bar{\delta}}(\Omega)\right): \quad \mathrm{K}\left(\bar{\vartheta}_{\tau_{k}}\right) \nabla \bar{\vartheta}_{\tau_{k}} \rightarrow \eta \text { in } L^{1+\bar{\delta}}\left(0, T ; L^{1+\bar{\delta}}(\Omega)\right) .
$$


In order to identify the weak limit $\eta$, it is sufficient to observe that (cf. [35]) $\mathrm{K}\left(\bar{\vartheta}_{\tau_{k}}\right) \nabla \bar{\vartheta}_{\tau_{k}}=\nabla \widehat{\mathrm{K}}\left(\bar{\vartheta}_{\tau_{k}}\right)$ a.e. in $\Omega \times(0, T)$. Combining the growth property (2.17) of $\mathrm{K}$ (where $1 \leq \kappa<5 / 3$ ), with the strong convergence (5.13) of $\bar{\vartheta}_{\tau_{k}}$ in $L^{p}(Q)$ for all $1 \leq p<8 / 3$, we ultimately conclude that $\left(\widehat{\mathrm{K}}\left(\bar{\vartheta}_{\tau_{k}}\right)\right)_{k}$ strongly converges to $\widehat{\mathrm{K}}(\vartheta)$ in $L^{1+\tilde{\delta}}(Q)$ for some $\tilde{\delta}>0$. A standard argument then yields

$$
\eta=\nabla \widehat{\mathrm{K}}(\vartheta)=\mathrm{K}(\vartheta) \nabla \vartheta \quad \text { a.e. in } \Omega \times(0, T) .
$$

Combining (5.35) and (5.36) leads to

$$
\int_{0}^{T} \int_{\Omega} \mathrm{K}\left(\bar{\vartheta}_{\tau_{k}}\right) \nabla \bar{\vartheta}_{\tau_{k}} \cdot \nabla \varphi \mathrm{d} x \mathrm{~d} t \rightarrow \int_{0}^{T} \int_{\Omega} \mathrm{K}(\vartheta) \nabla \vartheta \cdot \nabla \varphi \mathrm{d} x \mathrm{~d} t
$$

for every test function $\varphi \in \mathrm{C}^{0}\left([0, T] ; W^{2, d+\epsilon}(\Omega)\right)$.

To complete the passage to the limit on the right-hand side of (4.55), it remains to show that

$$
\partial_{t} \chi_{\tau_{k}} \rightarrow \chi_{t} \quad \text { in } L^{2}\left(0, T ; L^{2}(\Omega)\right) .
$$

This follows from testing the discrete equation for $\chi$ (4.57) by $\partial_{t} \chi_{\tau_{k}}$, integrating in time, and passing to the limit as $k \rightarrow \infty$. Indeed, exploiting convergences (5.2) and (5.6) $-(5.9)$ we deduce that

$$
\limsup _{k \rightarrow \infty} \int_{0}^{T} \int_{\Omega}\left|\partial_{t} \chi_{\tau_{k}}\right|^{2} \mathrm{~d} x \mathrm{~d} t \leq \int_{0}^{T} \int_{\Omega}\left|\chi_{t}\right|^{2} \mathrm{~d} x \mathrm{~d} t,
$$

whence (5.37).

In this way, we conclude that the limit triple $(\vartheta, \mathbf{u}, \chi)$ fulfills for all $t \in[0, T]$

$$
\begin{aligned}
& \langle\vartheta(t), \varphi(t)\rangle_{W^{2, d+\epsilon}(\Omega)}-\int_{0}^{t} \int_{\Omega} \vartheta \varphi_{t} \mathrm{~d} x \mathrm{~d} s+\int_{0}^{t} \int_{\Omega} \chi_{t} \vartheta \varphi \mathrm{d} x \mathrm{~d} s+\rho \int_{0}^{t} \int_{\Omega} \operatorname{div}\left(\mathbf{u}_{t}\right) \vartheta \varphi \mathrm{d} x \mathrm{~d} s \\
& \quad+\int_{0}^{t} \int_{\Omega} \mathrm{K}(\vartheta) \nabla \vartheta \nabla \varphi \mathrm{d} x \mathrm{~d} s=\int_{0}^{t} \int_{\Omega}\left(g+\frac{\varepsilon\left(\mathbf{u}_{t}\right) \mathbb{V} \varepsilon\left(\mathbf{u}_{t}\right)}{2}+\left|\chi_{t}\right|^{2}\right) \varphi \mathrm{d} x \mathrm{~d} s+\int_{0}^{t} \int_{\partial \Omega} h \varphi \mathrm{d} S \mathrm{~d} s+\int_{\Omega} \vartheta_{0} \varphi(0) \mathrm{d} x \\
& \quad \text { for all } \varphi \in C^{0}\left([0, T] ; W^{2, d+\epsilon}(\Omega)\right) \cap H^{1}\left(0, T ; L^{6 / 5}(\Omega)\right) \text { for some } \epsilon>0,
\end{aligned}
$$

whence for every $\bar{\varphi} \in W^{2, d+\epsilon}(\Omega)$ and for every $0 \leq s \leq t \leq T$

$$
\begin{aligned}
\langle\vartheta(t)-\vartheta(s), \bar{\varphi}\rangle_{W^{2, d+\epsilon}(\Omega)}= & -\int_{s}^{t} \int_{\Omega} \chi_{t} \vartheta \bar{\varphi} \mathrm{d} x \mathrm{~d} r-\rho \int_{s}^{t} \int_{\Omega} \operatorname{div}\left(\mathbf{u}_{t}\right) \vartheta \bar{\varphi} \mathrm{d} x \mathrm{~d} r-\int_{s}^{t} \int_{\Omega} \mathrm{K}(\vartheta) \nabla \vartheta \nabla \bar{\varphi} \mathrm{d} x \mathrm{~d} r \\
& +\int_{s}^{t} \int_{\Omega}\left(g+\frac{\varepsilon\left(\mathbf{u}_{t}\right) \mathbb{V} \varepsilon\left(\mathbf{u}_{t}\right)}{2}+\left|\chi_{t}\right|^{2}\right) \bar{\varphi} \mathrm{d} x \mathrm{~d} r+\int_{s}^{t} \int_{\partial \Omega} h \bar{\varphi} \mathrm{d} S \mathrm{~d} r .
\end{aligned}
$$

Then, we deduce from (5.39) that $\vartheta$ is absolutely continuous with values in $W^{2, d+\epsilon}(\Omega)^{*}$. Thus, we recover the improved regularity (2.56), and the improved formulation of the heat equation

$$
\begin{aligned}
& \int_{0}^{t}\left\langle\partial_{t} \vartheta, \varphi\right\rangle_{W^{2, d+\epsilon}(\Omega)} \mathrm{d} s+\int_{0}^{t} \int_{\Omega} \chi_{t} \vartheta \varphi \mathrm{d} x \mathrm{~d} s+\rho \int_{0}^{t} \int_{\Omega} \operatorname{div}\left(\mathbf{u}_{t}\right) \vartheta \varphi \mathrm{d} x \mathrm{~d} s \\
& \quad+\int_{0}^{t} \int_{\Omega} \mathrm{K}(\vartheta) \nabla \vartheta \nabla \varphi \mathrm{d} x \mathrm{~d} s=\int_{0}^{t} \int_{\Omega}\left(g+\frac{\varepsilon\left(\mathbf{u}_{t}\right) \mathbb{V} \varepsilon\left(\mathbf{u}_{t}\right)}{2}+\left|\chi_{t}\right|^{2}\right) \varphi \mathrm{d} x \mathrm{~d} s+\int_{0}^{t} \int_{\partial \Omega} h \varphi \mathrm{d} S \mathrm{~d} s
\end{aligned}
$$

for all $\varphi \in C^{0}\left([0, T] ; W^{2, d+\epsilon}(\Omega)\right)$ for some $\epsilon>0$ and for all $t \in[0, T]$.

Clearly, from (5.40) we obtain (2.57) by differentiating in time.

The total energy equality (2.58), holding for every $0 \leq s \leq t \leq T$, ensues from testing (2.57) by $\varphi=1$, the momentum balance (2.42) by $\mathbf{u}_{t}$, and the (pointwise) $\chi$-equation (2.53) by $\chi_{t}$, adding the resulting relations, and integrating in time. 


\section{From the $p$-Laplacian to the Laplacian}

In this Section we prove a global-in-time existence result for a suitable entropic formulation of the initialboundary value problem for system (1.1)-(1.3), in the case the $p$-Laplacian operator $-\operatorname{div}\left(|\nabla \chi|^{p-1} \nabla \chi\right)$ is replaced by the Laplacian $-\Delta \chi$, i.e. for $p=2$, keeping the evolution unidirectional (i.e., $\mu=1$ ). Hence, (1.3) rewrites as

$$
\chi_{t}+\partial I_{(-\infty, 0]}\left(\chi_{t}\right)-\Delta \chi+W^{\prime}(\chi) \ni-b^{\prime}(\chi) \frac{\varepsilon(\mathbf{u}) \mathbb{E} \varepsilon(\mathbf{u})}{2}+\vartheta \quad \text { in } \Omega \times(0, T) .
$$

We restrict, apparently for technical reasons (which however we cannot bypass), to the irreversible case $\mu=1$. The main idea of the technique consists in passing to the limit as $\delta \searrow 0$ in the following approximation of (6.1)

$$
\chi_{t}+\partial I_{(-\infty, 0]}\left(\chi_{t}\right)-\Delta \chi-\delta \operatorname{div}\left(|\nabla \chi|^{p-1} \nabla \chi\right)+W^{\prime}(\chi) \ni-b^{\prime}(\chi) \frac{\varepsilon(\mathbf{u}) \mathbb{E} \varepsilon(\mathbf{u})}{2}+\vartheta \quad \text { in } \Omega \times(0, T) .
$$

Indeed, under suitable conditions the existence result in Thm. 1 applies to the initial-boundary value problem for system (1.1)-(1.2), (6.2), with $p>d$ (supplemented with the boundary conditions (1.4)), yielding the existence of global-in-time entropic solutions for fixed $\delta>0$. In this entropic formulation we will then pass to the limit as $\delta \searrow 0$, recovering an existence result for the case $p=2$. In what follows, we will in fact work under a set of assumptions suited to the limit passage as $\delta \searrow 0$, but slightly weaker than the ones necessary to apply the existence Theorem 1 cf. e.g. Remark 6.3

Let us now state the notion of entropic solution for the limit system as $\delta \rightarrow 0$. We mention in advance that the solution concept introduced below is weaker than the one we have obtained in the case $p>d$ (cf. Definition 2.5). In fact, the total energy inequality holds true only on $(0, t)$ (cf. (6.7) below), and not on a generic interval $(s, t)$, and so does the energy-dissipation energy inequality in the weak formulation of the equation for $\chi$. Moreover, the momentum equation is no longer formulated pointwise a.e. in $\Omega \times(0, T)$, but in $H^{-1}\left(\Omega ; \mathbb{R}^{d}\right)$, a.e. in time, only. Let us also anticipate that we will confine to initial data $\chi_{0} \in H^{1}(\Omega)$ such that $\chi_{0} \geq 0$ a.e. in $\Omega$ (which gives $\widehat{\beta}\left(\chi_{0}\right) \in L^{1}(\Omega)$ as in $(2.28)$ ) and, at the same time, $\chi_{0} \leq 1$ a.e. in $\Omega$. This and the irreversible character of the evolution will ensure that $\chi \in[0,1]$ a.e. in $\Omega \times(0, T)$, in accord with the physical meaning of $\chi$.

Definition 6.1 (Entropic solutions to the irreversible system with $p=2)$. Given initial data $\left(\vartheta_{0}, \mathbf{u}_{0}, \mathbf{v}_{0}, \chi_{0}\right)$ such that $\vartheta_{0}$ fulfills $(\underline{2.26}),\left(\mathbf{u}_{0}, \mathbf{v}_{0}\right) \in H_{0}^{1}\left(\Omega ; \mathbb{R}^{d}\right) \times L^{2}\left(\Omega ; \mathbb{R}^{d}\right)$, and $\chi_{0}$ such that

$$
\chi_{0} \in H^{1}(\Omega), \quad 0 \leq \chi_{0} \leq 1 \text { a.e. in } \Omega,
$$

we call a triple $(\vartheta, \mathbf{u}, \chi)$ an entropic solution to the Cauchy problem for system (1.1) -(1.2), (6.1) with the boundary conditions (1.4), if

$$
\begin{aligned}
& \vartheta \in L^{2}\left(0, T ; H^{1}(\Omega)\right) \cap L^{\infty}\left(0, T ; L^{1}(\Omega)\right), \\
& \mathbf{u} \in H^{1}\left(0, T ; H_{0}^{1}\left(\Omega ; \mathbb{R}^{d}\right)\right) \cap W^{1, \infty}\left(0, T ; L^{2}\left(\Omega ; \mathbb{R}^{d}\right)\right) \cap H^{2}\left(0, T ; H^{-1}\left(\Omega ; \mathbb{R}^{d}\right)\right), \\
& \chi \in L^{\infty}\left(0, T ; H^{1}(\Omega)\right) \cap H^{1}\left(0, T ; L^{2}(\Omega)\right),
\end{aligned}
$$

$(\vartheta, \mathbf{u}, \chi)$ complies with the initial conditions (2.37)-(2.38), and with the entropic formulation of (1.1)-(1.2), (6.1) consisting of

- the entropy inequality (2.39);

- the total energy inequality for almost all $t \in(0, T]$ :

$$
\mathscr{E}\left(\vartheta(t), \mathbf{u}(t), \mathbf{u}_{t}(t), \chi(t)\right) \leq \mathscr{E}\left(\vartheta_{0}, \mathbf{u}_{0}, \mathbf{v}_{0}, \chi_{0}\right)+\int_{0}^{t} \int_{\Omega} g \mathrm{~d} x \mathrm{~d} r+\int_{0}^{t} \int_{\partial \Omega} h \mathrm{~d} S \mathrm{~d} r+\int_{0}^{t} \int_{\Omega} \mathbf{f} \cdot \mathbf{u}_{t} \mathrm{~d} x \mathrm{~d} r
$$

where

$$
\mathscr{E}\left(\vartheta, \mathbf{u}, \mathbf{u}_{t}, \chi\right):=\int_{\Omega} \vartheta \mathrm{d} x+\frac{1}{2} \int_{\Omega}\left|\mathbf{u}_{t}\right|^{2} \mathrm{~d} x+\frac{1}{2} \mathrm{e}(b(\chi(t)) \mathbf{u}(t), \mathbf{u}(t))+\frac{1}{2} \int_{\Omega}|\nabla \chi|^{2} \mathrm{~d} x+\int_{\Omega} W(\chi) \mathrm{d} x
$$


- the momentum equation

$$
\mathbf{u}_{t t}+\mathcal{V}\left(a(\chi) \mathbf{u}_{t}\right)+\mathcal{E}(b(\chi) \mathbf{u})+\mathcal{C}_{\rho}(\vartheta)=\mathbf{f} \quad \text { in } H^{-1}\left(\Omega ; \mathbb{R}^{d}\right) \quad \text { a.e. in }(0, T),
$$

- the weak formulation of (6.1), viz.

$$
\begin{aligned}
& \chi_{t}(x, t) \leq 0 \quad \text { for a.a. }(x, t) \in \Omega \times(0, T), \\
& \int_{\Omega}\left(\chi_{t}(t) \psi+\nabla \chi(t) \cdot \nabla \psi+\xi(t) \psi+\gamma(\chi(t)) \psi+b^{\prime}(\chi(t)) \frac{\varepsilon(\mathbf{u}(t)) \mathbb{E} \varepsilon(\mathbf{u}(t))}{2} \psi-\vartheta(t) \psi\right) \mathrm{d} x \geq 0 \\
& \quad \text { for all } \psi \in W_{-}^{1,2}(\Omega) \cap L^{\infty}(\Omega), \quad \text { for a.a.t } \in(0, T),
\end{aligned}
$$

where $\xi \in \partial I_{[0,+\infty)}(\chi)$ in the sense that

$\xi \in L^{1}\left(0, T ; L^{1}(\Omega)\right) \quad$ and $\quad\langle\xi(t), \psi-\chi(t)\rangle_{W^{1,2}(\Omega)} \leq 0 \quad \forall \psi \in W_{+}^{1,2}(\Omega) \cap L^{\infty}(\Omega)$, for a.a.t $\in(0, T)$,

as well as the energy inequality for all $t \in(0, T]$ :

$$
\begin{aligned}
\int_{0}^{t} \int_{\Omega}\left|\chi_{t}\right|^{2} \mathrm{~d} x \mathrm{~d} r & +\int_{\Omega}\left(\frac{1}{2}|\nabla \chi(t)|^{2}+W(\chi(t))\right) \mathrm{d} x \\
& \leq \int_{\Omega}\left(\frac{1}{2}\left|\nabla \chi_{0}\right|^{2}+W\left(\chi_{0}\right)\right) \mathrm{d} x+\int_{0}^{t} \int_{\Omega} \chi_{t}\left(-b^{\prime}(\chi) \frac{\varepsilon(\mathbf{u}) \mathbb{E} \varepsilon(\mathbf{u})}{2}+\vartheta\right) \mathrm{d} x \mathrm{~d} r .
\end{aligned}
$$

We are in the position now to state the main existence result of this section.

Theorem 6.2 (Existence of entropic solutions, $\mu=1$ and $p=2$ ). Let $\Omega$ be a bounded connected domain with Lipschitz boundary. Assume Hypotheses (I)-(III) with

$$
b^{\prime}(x) \geq 0 \text { for all } x \in \mathbb{R},
$$

and, in addition, Hypothesis (IV) (i.e., $\widehat{\beta}=I_{[0,+\infty)}$ ), as well as conditions (2.23) -(2.27) on the data $\mathbf{f}, g$, $h$, $\vartheta_{0}, \mathbf{u}_{0}, \mathbf{v}_{0}$, and (6.3) on $\chi_{0}$. Then, there exists an entropic solution (in the sense of Definition 6.1) $(\vartheta, \mathbf{u}, \chi)$ to the initial-boundary value problem for system (1.1)-(1.2), (6.1), such that $\log (\vartheta)$ complies with (2.48), $\xi$ in (6.12) is given by (2.49) and $\vartheta$ satisfies the strict positivity property (2.50).

Remark 6.3. Let us note that in Thm. 6.2 we are able to deal with the case of a Lipschitz domain $\Omega$ and we do not need $\mathrm{C}^{2}$-regularity of $\Omega(2.15)$. The latter condition was exploited in the previous sections in order to perform the elliptic regularity estimate on $\mathbf{u}$ (cf. the Fifth estimate (3.26) ), which is not carried out here. Indeed the regularity requirement (6.5) on $\mathbf{u}$ we ask for in Definition 6.1, and prove in Theorem 6.2, is weaker than the one prescribed in Section 2 (cf., e.g., (2.35) ). Moreover, for the same reason, in this case we could also consider more general boundary conditions on $\mathbf{u}$ than the homogeneous Dirichlet (1.4): for example mixed Dirichlet-Neumann conditions could be taken into account, without any restriction on the geometry of the domain.

Proof. Let $\left(\vartheta_{\delta}, \mathbf{u}_{\delta}, \chi_{\delta}\right)$ be a suitable family of entropic solutions to the initial-boundary value problem for (1.1) -(1.2), supplemented with initial data $\left(\vartheta_{0}, \mathbf{u}_{0}, \mathbf{v}_{0}\right)$ fulfilling (2.26)-(2.27), and with a sequence of data $\left(\chi_{0}^{\delta}\right)_{\delta}$ such that

$$
\left(\chi_{0}^{\delta}\right)_{\delta} \subset W^{1, p}(\Omega), \quad 0 \leq \chi_{0}^{\delta}(x) \leq 1 \text { for all } x \in \Omega \text { for all } \delta>0, \quad \chi_{0}^{\delta} \rightarrow \chi_{0} \text { in } H^{1}(\Omega) .
$$

Observe that we cannot rigorously perform on the entropic formulation of (1.1)-(1.2) the a priori estimates in Section 3. Therefore we need to confine the discussion only to the entropic solutions which arise from the time-discretization scheme set up in Sec. 4. In the present framework (i.e. with $p=2$ and $\mu=1$, and no upper bound on $\kappa$, cf. Hypothesis $(\mathrm{V})$ ), the a priori estimates for the time-discrete solutions in Prop. 4.10 are inherited in the time-continuous limit by the entropic solutions, with the exception of those corresponding to the Fifth, the Seventh, and the Eighth a priori estimates in Sec. 3, cf. also Remark 3.1. Concerning the Sixth estimate, as pointed out in Sec.4.3 we are only able to render a surrogate of it (i.e. (4.74)) on the time-discrete 
level. Still, this provides sufficient information to pass to the limit, cf. Lemma 5.1. We shall exploit this also within the present proof.

The convergences from Lemma 5.1 combined with lower semicontinuity arguments indeed ensure that the strict positivity of $\vartheta_{\delta}$ (cf. (3.2)), as well as estimates (3.5), (3.14), (3.17), (3.19), (3.20), (3.32), hold with constants uniform w.r.t. $\delta$. Moreover, combining the fact that $\widehat{\beta}=I_{[0,+\infty)}$ with the unidirectional character of the evolution and with the fact that $\chi_{\delta}(0)=\chi_{0}^{\delta} \in[0,1]$ on $\Omega$, we infer that

$$
\exists C>0 \quad \forall \delta>0: \quad\left\|\chi_{\delta}\right\|_{L^{\infty}(Q)} \leq C .
$$

Therefore, repeating the compactness arguments in the proof of Lemma 5.1, based on the compactness results in 51] (cf. also Theorem A.5 in the Appendix), for every vanishing sequence $\delta_{k} \downarrow 0$ as $k \rightarrow \infty$ there exist a not relabeled subsequence and a triple $(\vartheta, \mathbf{u}, \chi)$, along which there holds as $k \rightarrow \infty$ :

$$
\begin{aligned}
& \vartheta_{\delta_{k}}-^{*} \vartheta \quad \text { in } L^{2}\left(0, T ; H^{1}(\Omega)\right), \\
& \mathbf{u}_{\delta_{k}} \boldsymbol{P}^{*} \mathbf{u} \text { in } H^{2}\left(0, T ; H^{-1}\left(\Omega ; \mathbb{R}^{d}\right)\right) \cap W^{1, \infty}\left(0, T ; L^{2}\left(\Omega ; \mathbb{R}^{d}\right)\right) \cap H^{1}\left(0, T ; H^{1}\left(\Omega ; \mathbb{R}^{d}\right)\right), \\
& \partial_{t} \mathbf{u}_{\delta_{k}} \rightarrow \partial_{t} \mathbf{u} \quad \text { in } L^{2}\left(0, T ; L^{2}\left(\Omega ; \mathbb{R}^{d}\right)\right), \\
& \chi_{\delta_{k}}-^{*} \chi \quad \text { in } H^{1}\left(0, T ; L^{2}(\Omega)\right) \cap L^{\infty}\left(0, T ; H^{1}(\Omega)\right), \\
& \chi_{\delta_{k}} \rightarrow \chi \quad \text { in } L^{h}(\Omega \times(0, T)) \text { for all } h \in[1,+\infty), \\
& \log \left(\vartheta_{\delta_{k}}\right) \rightarrow \log (\vartheta) \text { in } L^{2}\left(0, T ; L^{s}(\Omega)\right) \text { for all } s \in(1,6) \text { for } d=3 \text { and for all } s \in(1,+\infty) \text { for } d=2, \\
& \vartheta_{\delta_{k}} \rightarrow \vartheta \quad \text { in } L^{h}(\Omega \times(0, T)), \text { for every } h \in[1,8 / 3) \text { for } d=3 \text { and } h \in[1,3) \text { if } d=2,
\end{aligned}
$$

and in addition $\vartheta \in L^{\infty}\left(0, T ; L^{1}(\Omega)\right)$.

Now, in order to pass to the limit as $\delta \searrow 0$ we need to prove, in addition, that $\partial_{t} \mathbf{u}_{\delta_{k}} \rightarrow \partial_{t} \mathbf{u}$ strongly in $L^{2}\left(0, T ; H^{1}\left(\Omega ; \mathbb{R}^{d}\right)\right)$. Observe that, in the case of the $p$-Laplacian regularization for $\chi$, we were able to prove an additional the strong convergence for (the sequence approximating) $\partial_{t} \mathbf{u}$ in $L^{2}\left(0, T ; H^{1}\left(\Omega ; \mathbb{R}^{d}\right)\right.$ ). Our argument resulted from compactness arguments, relying on the Fifth a priori estimate (i.e. the elliptic regularity estimate on $\mathbf{u}$ ). The latter is no longer at our disposal, now. The argument we will develop in the following lines is instead direct, and strongly based on the irreversible character of our system.

Strong convergence of $\partial_{t} \mathbf{u}_{\delta_{k}}$ in $L^{2}\left(0, T ; H^{1}\left(\Omega ; \mathbb{R}^{d}\right)\right)$. Let us test the weak formulation (2.42) of momentum equation fulfilled by the approximate solutions $\left(\vartheta_{\delta_{k}}, \mathbf{u}_{\delta_{k}}, \chi_{\delta_{k}}\right)_{k}$, by $\partial_{t}\left(\mathbf{u}_{\delta_{k}}-\mathbf{u}\right)$, where $\mathbf{u}$ is the limit of $\left(\mathbf{u}_{\delta_{k}}\right)_{k}$ as in (6.18)-(6.19). We get

$$
\begin{aligned}
& 0=\int_{0}^{t} \int_{\Omega} \partial_{t t}^{2} \mathbf{u}_{\delta_{k}} \partial_{t}\left(\mathbf{u}_{\delta_{k}}-\mathbf{u}\right) \mathrm{d} x \mathrm{~d} s+\int_{0}^{t} \mathbf{v}\left(a\left(\chi_{\delta_{k}}\right) \partial_{t} \mathbf{u}_{\delta_{k}}, \partial_{t}\left(\mathbf{u}_{\delta_{k}}-\mathbf{u}\right)\right) \mathrm{d} s \\
& +\int_{0}^{t} \mathrm{e}\left(b\left(\chi_{\delta_{k}}\right) \mathbf{u}_{\delta_{k}}, \partial_{t}\left(\mathbf{u}_{\delta_{k}}-\mathbf{u}\right)\right) \mathrm{d} s-\rho \int_{0}^{t} \int_{\Omega} \vartheta_{\delta_{k}} \operatorname{div}\left(\partial_{t}\left(\mathbf{u}_{\delta_{k}}-\mathbf{u}\right)\right) \mathrm{d} x \mathrm{~d} s-\int_{0}^{t} \int_{\Omega} \mathbf{f} \partial_{t}\left(\mathbf{u}_{\delta_{k}}-\mathbf{u}\right) \mathrm{d} x \mathrm{~d} s=: \sum_{i=1}^{5} I_{i} .
\end{aligned}
$$

Let us now deal separately with the single integrals $I_{1}, \ldots, I_{5}$ :

$$
\begin{aligned}
I_{1}:= & \int_{0}^{t} \int_{\Omega} \partial_{t t}^{2} \mathbf{u}_{\delta_{k}} \partial_{t}\left(\mathbf{u}_{\delta_{k}}-\mathbf{u}\right) \mathrm{d} x \mathrm{~d} s=\int_{0}^{t} \int_{\Omega} \partial_{t t}^{2}\left(\mathbf{u}_{\delta_{k}}-\mathbf{u}\right) \partial_{t}\left(\mathbf{u}_{\delta_{k}}-\mathbf{u}\right) \mathrm{d} x \mathrm{~d} s \\
& +\int_{0}^{t}\left\langle\partial_{t t}^{2} \mathbf{u}, \partial_{t}\left(\mathbf{u}_{\delta_{k}}-\mathbf{u}\right)\right\rangle_{H^{1}\left(\Omega ; \mathbb{R}^{d}\right)} \mathrm{d} s \\
= & \frac{1}{2}\left\|\partial_{t}\left(\mathbf{u}_{\delta_{k}}-\mathbf{u}\right)(t)\right\|_{L^{2}\left(\Omega ; \mathbb{R}^{d}\right)}^{2}-\frac{1}{2}\left\|\partial_{t}\left(\mathbf{u}_{\delta_{k}}-\mathbf{u}\right)(0)\right\|_{L^{2}\left(\Omega ; \mathbb{R}^{d}\right)}^{2}+\int_{0}^{t}\left\langle\partial_{t t}^{2} \mathbf{u}, \partial_{t}\left(\mathbf{u}_{\delta_{k}}-\mathbf{u}\right)\right\rangle_{H^{1}\left(\Omega ; \mathbb{R}^{d}\right)} \mathrm{d} s
\end{aligned}
$$


and the third integral tends to 0 when $\delta_{k} \searrow 0$ due to (6.18). Moreover,

$$
\begin{aligned}
I_{2}: & =\int_{0}^{t} \mathbf{v}\left(a\left(\chi_{\delta_{k}}\right) \partial_{t} \mathbf{u}_{\delta_{k}}, \partial_{t}\left(\mathbf{u}_{\delta_{k}}-\mathbf{u}\right)\right) \mathrm{d} s \\
& =\int_{0}^{t} \mathbf{v}\left(a\left(\chi_{\delta_{k}}\right) \partial_{t}\left(\mathbf{u}_{\delta_{k}}-\mathbf{u}\right), \partial_{t}\left(\mathbf{u}_{\delta_{k}}-\mathbf{u}\right)\right) \mathrm{d} s+\int_{0}^{t} \mathrm{v}\left(a\left(\chi_{\delta_{k}}\right) \partial_{t} \mathbf{u}, \partial_{t}\left(\mathbf{u}_{\delta_{k}}-\mathbf{u}\right)\right) \mathrm{d} s .
\end{aligned}
$$

Now, observe that

$$
a\left(\chi_{\delta_{k}}\right) \partial_{t} \mathbf{u} \rightarrow a(\chi) \partial_{t} \mathbf{u} \quad \text { in } L^{2}\left(0, T ; H^{1}\left(\Omega ; \mathbb{R}^{d}\right)\right)
$$

This follows from the fact that $a\left(\chi_{\delta_{k}}\right) \mathbf{u}_{t} \rightarrow a(\chi) \mathbf{u}_{t}$ and $a\left(\chi_{\delta_{k}}\right) \varepsilon\left(\mathbf{u}_{t}\right) \rightarrow a(\chi) \varepsilon\left(\mathbf{u}_{t}\right)$ a.e. in $\Omega \times(0, T)$, in view of convergence (6.21) and of the continuity of $a$. Moreover, also due to (6.16), we have that $\left\|a\left(\chi_{\delta_{k}}\right) \mathbf{u}_{t}\right\|_{H^{1}\left(\Omega ; \mathbb{R}^{d}\right)} \leq$ $C\left\|\mathbf{u}_{t}\right\|_{H^{1}\left(\Omega ; \mathbb{R}^{d}\right)}$ for a constant independent of $k \in \mathbb{N}$. Therefore, using the Lebesgue theorem the desired convergence (6.24) ensues. This implies that $\int_{0}^{t} \mathbf{v}\left(a\left(\chi_{\delta_{k}}\right) \partial_{t} \mathbf{u}, \partial_{t}\left(\mathbf{u}_{\delta_{k}}-\mathbf{u}\right)\right) \mathrm{d} s$ tends to 0 when $\delta_{k} \searrow 0$, due to (6.18). Integrating by parts in time, we get

$$
\begin{aligned}
I_{3}:= & \int_{0}^{t} \mathrm{e}\left(b\left(\chi_{\delta_{k}}\right) \mathbf{u}_{\delta_{k}}, \partial_{t}\left(\mathbf{u}_{\delta_{k}}-\mathbf{u}\right)\right) \mathrm{d} s \\
= & \int_{0}^{t} \mathrm{e}\left(b\left(\chi_{\delta_{k}}\right)\left(\mathbf{u}_{\delta_{k}}-\mathbf{u}\right), \partial_{t}\left(\mathbf{u}_{\delta_{k}}-\mathbf{u}\right)\right) \mathrm{d} s+\int_{0}^{t} \mathrm{e}\left(b\left(\chi_{\delta_{k}}\right) \mathbf{u}, \partial_{t}\left(\mathbf{u}_{\delta_{k}}-\mathbf{u}\right)\right) \mathrm{d} s \\
= & -\int_{0}^{t} \int_{\Omega} b^{\prime}\left(\chi_{\delta_{k}}\right) \partial_{t} \chi_{\delta_{k}} \frac{\varepsilon\left(\mathbf{u}_{\delta_{k}}-\mathbf{u}\right) \mathbb{E} \varepsilon\left(\mathbf{u}_{\delta_{k}}-\mathbf{u}\right)}{2} \mathrm{~d} x \mathrm{~d} s+\frac{1}{2} \mathrm{e}\left(b\left(\chi_{\delta_{k}}(t)\left(\mathbf{u}_{\delta_{k}}-\mathbf{u}\right)(t),\left(\mathbf{u}_{\delta_{k}}-\mathbf{u}\right)(t)\right)\right. \\
& -\frac{1}{2} \mathrm{e}\left(b\left(\chi_{\delta_{k}}(0)\right)\left(\mathbf{u}_{\delta_{k}}-\mathbf{u}\right)(0),\left(\mathbf{u}_{\delta_{k}}-\mathbf{u}\right)(0)\right)+\int_{0}^{t} \mathrm{e}\left(b\left(\chi_{\delta_{k}}\right) \mathbf{u}, \partial_{t}\left(\mathbf{u}_{\delta_{k}}-\mathbf{u}\right)\right) \mathrm{d} s
\end{aligned}
$$

where the last integral tends to 0 (this can be shown arguing in the same way as for the last term contributing to $I_{2}$ ), while the first integral is non-negative due to the fact that $\partial_{t} \chi_{\delta_{k}} \leq 0$ a.e. on $\Omega \times(0, T)$ and that $b^{\prime} \geq 0$. This is the point where we exploit the unidirectional character of the system (i.e. $\mu=1$ ). Finally,

$$
I_{4}:=-\int_{0}^{t} \int_{\Omega} \vartheta_{\delta_{k}} \varepsilon\left(\partial_{t}\left(\mathbf{u}_{\delta_{k}}-\mathbf{u}\right)\right) \mathrm{d} x \mathrm{~d} s \rightarrow 0, \quad I_{5}:=-\int_{0}^{t} \int_{\Omega} \mathbf{f} \partial_{t}\left(\mathbf{u}_{\delta_{k}}-\mathbf{u}\right) \mathrm{d} x \mathrm{~d} s \rightarrow 0,
$$

as $\delta_{k} \searrow 0$, due to the convergences (6.18), (6.23), as well as assumption (2.23) on $\mathbf{f}$. Ultimately, we get

$$
\left\|\partial_{t}\left(\mathbf{u}_{\delta_{k}}-\mathbf{u}\right)(t)\right\|_{L^{2}\left(\Omega ; \mathbb{R}^{d}\right)}^{2}+\int_{0}^{t} \mathrm{v}\left(a\left(\chi_{\delta_{k}}\right) \partial_{t}\left(\mathbf{u}_{\delta_{k}}-\mathbf{u}\right), \partial_{t}\left(\mathbf{u}_{\delta_{k}}-\mathbf{u}\right)\right) \mathrm{d} s+\mathrm{e}\left(b\left(\chi_{\delta_{k}}(t)\left(\mathbf{u}_{\delta_{k}}-\mathbf{u}\right)(t),\left(\mathbf{u}_{\delta_{k}}-\mathbf{u}\right)(t)\right) \rightarrow 0\right.
$$

as $\delta_{k} \searrow 0$, which entails

$$
\mathbf{u}_{\delta_{k}} \rightarrow \mathbf{u} \quad \text { strongly in } W^{1, \infty}\left(0, T ; L^{2}\left(\Omega ; \mathbb{R}^{d}\right)\right) \cap H^{1}\left(0, T ; H^{1}\left(\Omega ; \mathbb{R}^{d}\right)\right) .
$$

Conclusion of the proof. Using this strong convergence, we can now pass to the limit as $k \rightarrow \infty$ in the energy-dissipation inequality (2.44) featuring in the weak formulation of the equation for $\chi_{\delta_{k}}$ as follows. We have to identify the weak limit of

$$
\xi_{\delta_{k}}(x, t)=-\mathcal{J}_{\left\{\chi_{\delta_{k}}=0\right\}}(x, t)\left(\gamma\left(\chi_{\delta_{k}}(x, t)\right)+b^{\prime}\left(\chi_{\delta_{k}}(x, t)\right) \frac{\varepsilon\left(\mathbf{u}_{\delta_{k}}(x, t)\right) \mathbb{E}(x) \varepsilon\left(\mathbf{u}_{\delta_{k}}(x, t)\right)}{2}-\vartheta_{\delta_{k}}(x, t)\right)^{+} .
$$

First of all note that $\left(\mathcal{J}_{\left\{\chi_{\delta_{k}}=0\right\}}\right)_{k}$ is bounded in $L^{\infty}(Q)$ independently of $k \in \mathbb{N}$. Hence, we can select a subsequence $\left(\mathcal{J}_{\left\{\chi_{\delta_{k}}=0\right\}}\right)_{k}$ weakly star converging in $L^{\infty}(Q)$ to some $\mathcal{J}$. Observe that we cannot establish that $\mathcal{J}=\mathcal{J}_{\{\chi=0\}}$. On the other hand, it follows from the previously proved convergences that $\left(\gamma\left(\chi_{\delta_{k}}\right)+\right.$ $\left.b^{\prime}\left(\chi_{\delta_{k}}\right) \frac{\varepsilon\left(\mathbf{u}_{\delta_{k}}\right) \mathbb{E} \varepsilon\left(\mathbf{u}_{\delta_{k}}\right)}{2}-\vartheta_{\delta_{k}}\right)^{+}$strongly converges in $L^{1}(Q)$ to $\left(\gamma(\chi)+b^{\prime}(\chi) \frac{\varepsilon(\mathbf{u}) \mathbb{E} \varepsilon(\mathbf{u})}{2}-\vartheta\right)^{+}$. Hence we identify

$$
\xi=-\mathcal{J}(x, t)\left(\gamma(\chi(x, t))+b^{\prime}(\chi(x, t)) \frac{\varepsilon(\mathbf{u}(x, t)) \mathbb{E} \varepsilon(\mathbf{u}(x, t))}{2}-\vartheta(x, t)\right)^{+}
$$


and observe that $\xi_{\delta_{k}} \rightarrow \xi$ in $L^{1}(Q)$. Then, integrating (2.44) $\delta_{k}$ from 0 to $T$ and passing to the limit as $k \rightarrow \infty$, using the fact that or all $\psi \in L^{p}\left(0, T ; W_{-}^{1, p}(\Omega)\right) \cap L^{\infty}(Q)$

$$
\left.\left|\int_{0}^{T} \int_{\Omega} \delta_{k}\right| \nabla \chi_{\delta_{k}}\right|^{p-2} \nabla \chi_{\delta_{k}} \cdot \nabla \psi \mathrm{d} x \mathrm{~d} t \mid \leq \delta_{k}\left\|\nabla \chi_{\delta_{k}}\right\|_{L^{p-1}\left(Q ; \mathbb{R}^{d}\right)}^{p-1}\|\nabla \psi\|_{L^{p}\left(Q ; \mathbb{R}^{d}\right)} \rightarrow 0
$$

we get

$$
\int_{0}^{T} \int_{\Omega}\left(\chi_{t}(t) \psi+\nabla \chi(t) \cdot \nabla \psi+\gamma(\chi(t)) \psi+b^{\prime}(\chi(t)) \frac{\varepsilon(\mathbf{u}(t)) \mathbb{E} \varepsilon(\mathbf{u}(t))}{2} \psi-\vartheta(t) \psi\right) \mathrm{d} x \mathrm{~d} t \geq-\int_{0}^{T} \int_{\Omega} \xi(t) \psi \mathrm{d} x \mathrm{~d} t
$$

for all $\psi \in L^{p}\left(0, T ; W_{-}^{1, p}(\Omega)\right) \cap L^{\infty}(Q)$, where $\xi$ is defined in (6.27). From (6.28), we get (6.11).

It remains to show that $\chi$ complies with the variational inequality (6.12). To do so, we have to pass to the limit in (2.45) $\delta_{k}$, whence we have

$$
\int_{0}^{T}\left(\int_{\Omega} \xi_{\delta_{k}}\left(\psi-\chi_{\delta_{k}}(t)\right) \mathrm{d} x\right) \zeta(t) \mathrm{d} t \geq 0 \quad \text { for all } \psi \in W_{+}^{1, p}(\Omega) \text { and all } \zeta \in L^{\infty}(0, T) \text { with } \psi, \zeta \geq 0 .
$$

Observe that the two weak convergences $\chi_{\delta_{k}} \rightarrow^{*} \chi$ in $L^{\infty}(Q)$ and $\xi_{\delta_{k}} \rightarrow \xi$ in $L^{1}(Q)$ do not allow for a direct limit passage in the term $\iint_{Q} \xi_{\delta_{k}} \chi_{\delta_{k}} \zeta \mathrm{d} x \mathrm{~d} t$, which equals zero for all $k \in \mathbb{N}$ due to (6.26). Indeed, we need to argue in a more refined way. It follows from (6.21) that $\chi_{\delta_{k}}$ converges almost uniformly to $\chi$ in $Q$, i.e. for every $\epsilon>0$ there exists $Q_{\epsilon} \subset Q$ such that $\left|Q \backslash Q_{\epsilon}\right|<\epsilon$ and $\chi_{\delta_{k}} \rightarrow \chi$ uniformly on $Q_{\epsilon}$. The latter property implies that

$$
\mathcal{J} \equiv 0 \text { on } Q_{\epsilon} \cap\left\{\mathcal{J}_{\{\chi=0\}} \equiv 0\right\}
$$

Indeed, $\mathcal{J}_{\{\chi=0\}}(x, t)=0$ implies $\chi(x, t) \neq 0$. Since $\chi_{\delta_{k}}$ converges to $\chi$ uniformly on $Q_{\epsilon}$, there exists an index $\bar{k}$, independent of $(x, t)$, such that for all $k \geq \bar{k}, \chi_{\delta_{k}}(x, t) \neq 0$, hence $\mathcal{J}_{\left\{\chi_{\delta_{k}}=0\right\}}(x, t)=0$. With this argument we conclude that $\mathcal{J}_{\left\{\chi_{\delta_{k}}=0\right\}} \equiv 0$ on $Q_{\epsilon} \cap\left\{\mathcal{J}_{\left\{\chi_{=0\}}\right.} \equiv 0\right\}$, whence (6.29). It follows from (6.29) and (6.27) that

$$
\xi(x, t) \chi(x, t)=0 \quad \text { for a.a. }(x, t) \in Q_{\epsilon}, \text { whence } \iint_{Q_{\epsilon}} \xi(x, t) \chi(x, t) \zeta(t) \mathrm{d} x \mathrm{~d} t=0 .
$$

On the other hand, using the properties of the Lebesgue integral we have that

$$
\forall \eta>0 \quad \exists \epsilon=\epsilon_{\eta}>0:\left|Q \backslash Q_{\epsilon}\right|<\epsilon \Rightarrow \iint_{Q \backslash Q_{\epsilon}}|\xi(x, t) \chi(x, t) \zeta(t)| \mathrm{d} x \mathrm{~d} t<\eta .
$$

Therefore we conclude that

$$
\forall \eta>0 \quad\left|\iint_{Q} \xi(x, t) \chi(x, t) \zeta(t) \mathrm{d} x \mathrm{~d} t\right|<\eta
$$

i.e.

$$
\iint_{Q} \xi(x, t) \chi(x, t) \zeta(t) \mathrm{d} x \mathrm{~d} t=0=\lim _{k \rightarrow \infty} \iint_{Q} \xi_{\delta_{k}} \chi_{\delta_{k}} \zeta \mathrm{d} x \mathrm{~d} t
$$

Hence

$$
0 \leq \iint_{Q} \xi_{\delta_{k}}\left(\psi-\chi_{\delta_{k}}\right) \zeta \mathrm{d} x \mathrm{~d} t \rightarrow \iint_{Q} \xi(\psi-\chi) \zeta \mathrm{d} x \mathrm{~d} t=\int_{0}^{T}\left(\int_{\Omega} \xi(\psi-\chi(t)) \mathrm{d} x\right) \zeta(t) \mathrm{d} t
$$

which implies

$$
\int_{\Omega} \xi(t)(\psi-\chi(t)) \mathrm{d} x \geq 0 \quad \text { for a.e. } t \in(0, T) \quad \text { for all } \psi \in W_{+}^{1, p}(\Omega) .
$$

With a density argument we get (6.12) for all $\psi \in W_{+}^{1,2}(\Omega) \cap L^{\infty}(\Omega)$.

Convergences (6.17)-(6.23) also guarantee the passage to the limit in the momentum equation, whence (6.9).

Finally, we pass to the limit in the entropy inequality (2.39) and in the total energy inequality (2.40) by the very same compactness/lower semicontinuity arguments as in the proof of Theorem 1) thus deducing (2.39) and the total energy inequality (6.7) on the generic interval $(0, t)$. 
Remark 6.4. Notice that, we have been able to obtain the energy inequalities (6.13) and (6.8) only on intervals of the type $(0, t)$, and not on the generic interval $(s, t) \subset(0, T)$, due to the weak convergence of $\left(\nabla \chi_{\delta_{k}}\right)$ in $L^{2}\left(Q ; \mathbb{R}^{d}\right)$, which does not yield the pointwise-in-time convergence required to take the limit of the right-hand sides of (2.46) and (2.40). It is an open problem to improve the convergence of $\left(\nabla \chi_{\delta_{k}}\right)$ to a strong one.

This limit passage also reveals that the notion of entropic solution enjoys stability properties. It seems to be the right one in the present framework, and, apparently, the entropy inequality cannot be improved to a suitable variational formulation of the heat equation like in the case of Theorem 2, at least with these techniques.

\section{Appendix A. Auxiliary compactness Results}

The main compactness result of this Appendix, Theorem A.5 below, hinges on a compactness argument drawn from the theory of parameterized (or Young) measures with values in an infinite-dimensional space.

Hence, for the reader's convenience, we preliminarily collect here the definition of Young measure with values in a reflexive Banach space $X$. We then recall the Young measure compactness result from [36, which was proved in [44] in the case when $X$ is a Hilbert space, extending to the frame of the weak topology classical results within Young measure theory (see e.g. [3, Thm. 1], 4] [52, Thm. 16]).

We start by fixing some

Notation A.1. Given an interval $I \subset \mathbb{R}$, we denote by $\mathscr{L}_{I}$ the $\sigma$-algebra of the Lebesgue measurable subsets of $I$ and, given a reflexive Banach space $X$, by $\mathscr{B}(X)$ its Borel $\sigma$-algebra.

Definition A.2 ((Time-dependent) Young measures). A Young measure in the space $X$ is a family $\boldsymbol{\mu}:=\left\{\mu_{t}\right\}_{t \in(0, T)}$ of Borel probability measures on $X$ such that the map on $(0, T)$

$$
t \mapsto \mu_{t}(A) \quad \text { is } \quad \mathscr{L}_{(0, T)} \text {-measurable for all } A \in \mathscr{B}(X) .
$$

We denote by $\mathscr{Y}(0, T ; X)$ the set of all Young measures in $X$.

The following result subsumes part of the statements of [36. Theorems A.2, A.3]: its crucial finding for our purposes concerns the characterization of the limit points in the weak topology of $L^{p}(0, T ; X), p \in(1,+\infty]$, of a bounded sequence $\left(\ell_{n}\right)_{n} \subset L^{p}(0, T ; X)$. Every limit point arises as the barycenter of the limiting Young measure $\boldsymbol{\mu}=\left(\mu_{t}\right)_{t \in(0, T)}$ associated with (a suitable subsequence $\left(\ell_{n_{k}}\right)_{k}$ of) $\left(\ell_{n}\right)_{n}$. In turn, for almost all $t \in(0, T)$ the support of the measure $\mu_{t}$ is concentrated in the set of limit points of $\left(\ell_{n_{k}}(t)\right)_{k}$ with respect to the weak topology of $X$. This information will play a crucial role in the proof of Theorem A.5 ahead.

Theorem A.3. [36, Theorems A.2, A.3] Let $p>1$ and let $\left(w_{n}\right)_{n} \subset L^{p}(0, T ; X)$ be a bounded sequence. Then, there exist a subsequence $\left(w_{n_{k}}\right)_{k}$ and a Young measure $\boldsymbol{\mu}=\left\{\mu_{t}\right\}_{t \in(0, T)}$ such that for a.a. $t \in(0, T)$

$$
\mu_{t} \text { is concentrated on the set } \bigcap_{p=1}^{\infty}{\overline{\left\{w_{n_{k}}(t): k \geq p\right\}}}^{\text {weak- } X}
$$

of the limit points of the sequence $\left(w_{n_{k}}(t)\right)$ with respect to the weak topology of $X$ and, setting

$$
w(t):=\int_{X} \omega \mathrm{d} \mu_{t}(\omega) \quad \text { for a.a.t } \in(0, T),
$$

there holds

$$
w_{n_{k}} \rightarrow w \quad \text { in } L^{p}(0, T ; X) \quad \text { as } k \rightarrow \infty
$$

with $\rightarrow$ replaced by $\rightarrow^{*}$ if $p=\infty$.

The statement of Theorem A.5 ahead features two reflexive Banach spaces $V$ and $Y$. Further, we will use the following 
Notation A.4. We denote by $\bar{B}_{1, Y}(0)$ the closed unitary ball in $Y$, and we will work with the space

$$
\mathrm{B}\left([0, T] ; Y^{*}\right):=\left\{\ell:[0, T] \rightarrow Y^{*}: \text { measurable, such that } \ell(t) \text { is defined at every } t \in[0, T]\right\} .
$$

Moreover, for given $\ell \in \mathrm{B}\left([0, T] ; Y^{*}\right), \varphi \in Y$, and $[a, b] \subset[0, T]$, we set

$$
\operatorname{Var}\left(\langle\ell, \varphi\rangle_{Y} ;[a, b]\right):=\sup \left\{\sum_{i=1}^{J}\left|\left\langle\ell\left(\sigma_{i}\right), \varphi\right\rangle_{Y}-\left\langle\ell\left(\sigma_{i-1}\right), \varphi\right\rangle_{Y}\right|: a=\sigma_{0}<\sigma_{1}<\ldots<\sigma_{J}=b\right\} .
$$

We are now in the position to state and prove the main result of this section, combining Thm. A.3 with ideas from [39, Thm. 6.1].

Theorem A.5. Let $V$ and $Y$ be two (separable) reflexive Banach spaces such that $V \subset Y^{*}$ continuously. Let $\left(\ell_{n}\right)_{n} \subset L^{p}(0, T ; V) \cap \mathrm{B}\left([0, T] ; Y^{*}\right)$ be bounded in $L^{p}(0, T ; V)$ and suppose in addition that

$$
\begin{aligned}
& \left(\ell_{n}(0)\right)_{n} \subset Y^{*} \text { is bounded, } \\
& \exists C>0 \quad \forall \varphi \in \bar{B}_{1, Y}(0) \quad \forall n \in \mathbb{N}: \quad \operatorname{Var}\left(\left\langle\ell_{n}, \varphi\right\rangle_{Y} ;[0, T]\right) \leq C .
\end{aligned}
$$

Then, there exists a subsequence $\left(\ell_{n_{k}}\right)_{k}$ of $\left(\ell_{n}\right)_{n}$ and a function $\ell \in L^{p}(0, T ; V) \cap L^{\infty}\left(0, T ; Y^{*}\right)$ such that as $k \rightarrow \infty$

$$
\begin{aligned}
& \ell_{n_{k}}-^{*} \ell \quad \text { in } L^{p}(0, T ; V) \cap L^{\infty}\left(0, T ; Y^{*}\right), \\
& \ell_{n_{k}}(t) \rightarrow \ell(t) \quad \text { in } V \quad \text { for a.a.t } \in(0, T) .
\end{aligned}
$$

Proof. We split the proof in two claims. For the first one, we closely follow the arguments from the proof of [39, Thm. 6.1].

Claim 1: Let $\mathcal{F} \subset \bar{B}_{1, Y}(0)$ be countable and dense in $\bar{B}_{1, Y}(0)$. There exist a subsequence $\left(\ell_{n_{k}}\right)_{k}$ of $\left(\ell_{n}\right)_{n}$, and for every $\varphi \in \mathcal{F}$ a function $\mathscr{L}_{\varphi}:[0, T] \rightarrow \mathbb{R}$ such that for every $\varphi \in \mathcal{F}$

$$
\left\langle\ell_{n_{k}}(t), \varphi\right\rangle_{Y} \rightarrow \mathscr{L}_{\varphi}(t) \quad \text { as } k \rightarrow \infty \quad \text { for every } t \in[0, T]
$$

With every $\varphi \in \bar{B}_{1, Y}(0)$ we may associate the monotone functions $\mathcal{V}_{n}^{\varphi}:[0, T] \rightarrow[0,+\infty)$ defined by $\mathcal{V}_{n}^{\varphi}(t):=$ $\operatorname{Var}\left(\left\langle\ell_{n}, \varphi\right\rangle_{Y} ;[0, t]\right)$ for every $t \in[0, T]$. Let now $\mathcal{F} \subset \bar{B}_{1, Y}(0)$ be countable and dense and let us consider the

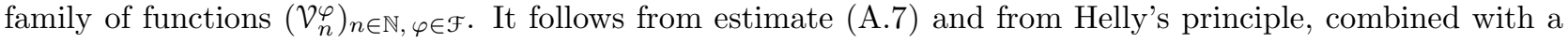
diagonalization procedure based on the countability of $\mathcal{F}$, that there exist a sequence of indexes $\left(n_{k}\right)_{k}$ and for every $\varphi \in \mathcal{F}$ a monotone function $\mathcal{V}_{\infty}^{\varphi}:[0, T] \rightarrow[0,+\infty)$ such that

$$
\mathcal{V}_{n_{k}}^{\varphi}(t) \rightarrow \mathcal{V}_{\infty}^{\varphi}(t) \quad \text { as } k \rightarrow \infty \text { for every } t \in[0, T] .
$$

Since $\mathcal{V}_{\infty}^{\varphi}$ is monotone, it has an at most countable jump set $J_{\varphi}$. The set $J:=\cup_{\varphi \in \mathcal{F}} J_{\varphi}$ is still countable, and therefore we may choose a sequence $\mathcal{T}:=\left(t_{m}\right)_{m} \subset[0, T]$, dense in $[0, T]$, such that $J \subset \mathcal{T}$.

Observe that (A.6) and (A.7) yield that

$$
\exists C>0 \quad \forall n \in \mathbb{N} \forall t \in[0, T]: \quad\left\|\ell_{n}(t)\right\|_{Y^{*}} \leq C .
$$

Since $Y^{*}$ is reflexive, with a diagonal argument we may extract a further, not relabeled, subsequence such that for every $t \in \mathcal{T}=\left(t_{m}\right)_{m}$

$$
\ell_{n_{k}}(t) \rightarrow \tilde{\ell}(t) \quad \text { in } Y^{*} \text { as } k \rightarrow \infty
$$

for some $\tilde{\ell}(t) \in Y^{*}$. We now show that for every $\varphi \in \mathcal{F}$ the map $t \mapsto\langle\tilde{\ell}(t), \varphi\rangle_{Y}$ is weakly continuous at every point $t \in \mathcal{T} \backslash J$. Indeed, for every $t_{1}, t_{2} \in \mathcal{T}$ with $t_{1} \leq t_{2}$ and for every $\varphi \in \mathcal{F}$ one has

$$
\begin{aligned}
& \left|\left\langle\tilde{\ell}\left(t_{1}\right)-\tilde{\ell}\left(t_{2}\right), \varphi\right\rangle_{Y}\right|=\lim _{k \rightarrow \infty}\left|\left\langle\ell_{n_{k}}\left(t_{1}\right)-\ell_{n_{k}}\left(t_{2}\right), \varphi\right\rangle_{Y}\right| \\
& \quad \leq \lim _{k \rightarrow \infty} \operatorname{Var}\left(\left\langle\ell_{n_{k}}, \varphi\right\rangle_{Y} ;\left[t_{1}, t_{2}\right]\right)=\lim _{k \rightarrow \infty} \mathcal{V}_{n_{k}}^{\varphi}\left(t_{2}\right)-\lim _{k \rightarrow \infty} \mathcal{V}_{n_{k}}^{\varphi}\left(t_{1}\right)=\mathcal{V}_{\infty}^{\varphi}\left(t_{2}\right)-\mathcal{V}_{\infty}^{\varphi}\left(t_{1}\right) .
\end{aligned}
$$


Therefore, for every $\varphi \in \mathcal{F}$ one extends the map $t \mapsto\langle\tilde{\ell}(t), \varphi\rangle_{Y}$ to all of $[0, T]$ by continuity and thus obtains a function $\mathscr{L}_{\varphi}:[0, T] \rightarrow \mathbb{R}$ such that

$$
\mathscr{L}_{\varphi}(t)=\langle\tilde{\ell}(t), \varphi\rangle_{Y} \quad \text { for every } t \in \mathcal{T} .
$$

Let us now prove that

$$
\left\langle\ell_{n_{k}}(t), \varphi\right\rangle_{Y} \rightarrow \mathscr{L}_{\varphi}(t) \quad \text { as } k \rightarrow \infty \quad \text { for every } t \in[0, T] \text { and } \varphi \in \mathcal{F} .
$$

In view of (A.13), we may assume $t \in[0, T] \backslash J$. Then, we have

$$
\begin{aligned}
& \left|\left\langle\ell_{n_{k}}(t), \varphi\right\rangle_{Y}-\mathscr{L}_{\varphi}(t)\right| \\
& \leq\left|\left\langle\ell_{n_{k}}(t), \varphi\right\rangle_{Y}-\left\langle\ell_{n_{k}}\left(t_{m}\right), \varphi\right\rangle_{Y}\right|+\left|\left\langle\ell_{n_{k}}\left(t_{m}\right), \varphi\right\rangle_{Y}-\left\langle\tilde{\ell}\left(t_{m}\right), \varphi\right\rangle_{Y}\right|+\left|\left\langle\tilde{\ell}\left(t_{m}\right), \varphi\right\rangle_{Y}-\mathscr{L}_{\varphi}(t)\right| \\
& \doteq \Delta_{1}+\Delta_{2}+\Delta_{3}
\end{aligned}
$$

with $t_{m}$ in the dense set $\mathcal{T}$ suitably chosen. Now, on account of (A.11) for every fixed $\varepsilon>0$ there exists $k_{\varepsilon}^{1} \in \mathbb{N}$ such that for $k \geq k_{\varepsilon}^{1}$ one has

$$
\Delta_{1} \leq\left|\mathcal{V}_{n_{k}}^{\varphi}(t)-\mathcal{V}_{n_{k}}^{\varphi}\left(t_{m}\right)\right| \leq\left|\mathcal{V}_{\infty}^{\varphi}(t)-\mathcal{V}_{\infty}^{\varphi}\left(t_{m}\right)\right|+\frac{\varepsilon}{4} .
$$

Using that $\mathscr{L}_{\varphi}(t)=\lim _{t_{j} \rightarrow t}\left\langle\tilde{\ell}\left(t_{j}\right), \varphi\right\rangle_{Y}$ for some $\left(t_{j}\right)_{j} \subset \mathcal{T}$ with $t_{j} \rightarrow t$, we also have

$$
\begin{aligned}
& \Delta_{3}=\left|\left\langle\tilde{\ell}\left(t_{m}\right), \varphi\right\rangle_{Y}-\lim _{j \rightarrow \infty}\left\langle\tilde{\ell}\left(t_{j}\right), \varphi\right\rangle_{Y}\right| \stackrel{(1)}{\leq} \lim _{j \rightarrow \infty}\left|\mathcal{V}_{\infty}^{\varphi}\left(t_{m}\right)-\mathcal{V}_{\infty}^{\varphi}\left(t_{j}\right)\right| \\
& \stackrel{(2)}{=}\left|\mathcal{V}_{\infty}^{\varphi}\left(t_{m}\right)-\mathcal{V}_{\infty}^{\varphi}(t)\right|,
\end{aligned}
$$

where (1) follows from (A.14) and (2) from the fact that $t \in[0, T] \backslash J$ is a is a continuity point of $\mathcal{V}_{\infty}^{\varphi}$. In view of the latter fact, we may choose $t_{m}$ sufficiently close to $t$ such that $\left|\mathcal{V}_{\infty}^{\varphi}(t)-\mathcal{V}_{\infty}^{\varphi}\left(t_{m}\right)\right| \leq \frac{\varepsilon}{4}$. Finally, in view of (A.13), there exists $k_{\varepsilon}^{2} \in \mathbb{N}$ such that $\Delta_{2} \leq \frac{\varepsilon}{4}$ for $k \geq k_{\varepsilon}^{2}$. All in all, we conclude that for $k \geq \max \left\{k_{\varepsilon}^{1}, k_{\varepsilon}^{2}\right\}$ we have $\left|\left\langle\ell_{n_{k}}(t), \varphi\right\rangle_{Y}-\mathscr{L}_{\varphi}(t)\right| \leq \varepsilon$, which yields (A.16).

Claim 2: Let $\left(\ell_{n_{k}}\right)_{k}$ be a (not relabeled) subsequence of the sequence from Claim 1, with which a limiting Young measure $\boldsymbol{\mu}=\left\{\mu_{t}\right\}_{t \in(0, T)}$ is associated according to Theorem A.3. Then, for almost all $t \in(0, T)$ the probability measure $\mu_{t}$ is a Dirac mass $\delta_{\ell(t)}$, and (A.9) holds as $k \rightarrow \infty$.

In order to show that $\mu_{t}$ is a Dirac mass, we are going to prove that the set of the limit points of $\left(\ell_{n_{k}}(t)\right)_{k}$ in the weak topology of $V$ is a singleton. To this aim, let us pick two points $\ell_{\infty}^{1}, \ell_{\infty}^{2} \in \bigcap_{p=1}^{\infty}{\overline{\left\{\ell_{n_{k}}(t): k \geq p\right\}}}^{\text {weak-V}}$, and two subsequences $\left(\ell_{n_{k}^{1}}(t)\right)_{k},\left(\ell_{n_{k}^{2}}(t)\right)_{k}$, possibly depending on $t$, such that $\ell_{n_{k}^{i}}(t) \rightarrow \ell_{\infty}^{i}$ in $V$ as $k \rightarrow \infty$ for $i=1,2$. Then $\ell_{n_{k}^{i}}(t) \rightarrow \ell_{\infty}^{i}$ in $Y^{*}$. In view of (A.10) conclude that

$$
\left\langle\ell_{\infty}^{1}(t), \varphi\right\rangle_{Y}=\mathscr{L}_{\varphi}(t)=\left\langle\ell_{\infty}^{2}(t), \varphi\right\rangle_{Y} \quad \text { for every } \varphi \in \mathcal{F} .
$$

Since $\mathcal{F}$ is dense in $\bar{B}_{1, Y}(0)$, we deduce that $\ell_{\infty}^{1}$ and $\ell_{\infty}^{2}$ coincide on all the elements in $\bar{B}_{1, Y}(0)$. But then by linearity we have that $\ell_{\infty}^{1}=\ell_{\infty}^{2}$ in $Y^{*}$, hence in $V$. Therefore for almost all $t \in(0, T)$ the set $\bigcap_{p=1}^{\infty}{\overline{\left\{\ell_{n_{k}}(t): k \geq p\right\}}}^{\text {weak } V}$ is a singleton $\{\ell(t)\}$, and (A.9) ensues. Observe that the functions $\ell$ and $\tilde{\ell}$ from Claim 1 need not coincide, as (A.15) only holds on the set $\mathcal{T}$ with zero Lebesgue measure.

Finally, (A.8) results from (A.3) taking into account that $\ell(t)=\int_{V} l \mathrm{~d} \delta_{\ell(t)}(l)$ and that the sequence $\left(\ell_{n}\right)$ is also bounded in $L^{\infty}\left(0, T ; Y^{*}\right)$, cf. (A.12).

Acknowledgments. The work of Elisabetta Rocca was supported by the FP7-IDEAS-ERC-StG Grant \#256872 (EntroPhase) and by GNAMPA (Gruppo Nazionale per l'Analisi Matematica, la Probabilità e le loro Applicazioni) of INdAM (Istituto Nazionale di Alta Matematica). Riccarda Rossi was partially supported by a MIUR-PRIN'10-'11 grant for the project "Calculus of Variations", and by GNAMPA (Gruppo Nazionale per l'Analisi Matematica, la Probabilità e le loro Applicazioni) of INdAM (Istituto Nazionale di Alta Matematica). The authors thank the anonymous referees for their careful reading of the manuscript, which greatly improved the quality of the paper. 


\section{REFERENCES}

[1] H. Abels: Existence of weak solutions for a diffuse interface model for viscous, incompressible fluids with general densities, Comm. Math. Phys. 289 (2009), 45-73.

[2] G. Akagi: Maximal monotonicity for the sum of two subdifferential operators in $L^{p}$-spaces, Nonlinear Anal. 74 (2011), $1664-1671$.

[3] E.J. Balder: A general approach to lower semicontinuity and lower closure in optimal control theory, SIAM J. Control Optim. 22 (1984), 570-598.

[4] J.M. Ball: A version of the fundamental theorem for Young measures, in PDEs and continuum models of phase transitions (Nice, 1988), vol. 344 of Lecture Notes in Phys, pp. 207-215. Springer, Berlin (1989).

[5] E. Bonetti, G. Bonfanti: Well-posedness results for a model of damage in thermoviscoelastic materials, Ann. Inst. H. Poincaré Anal. Non Linéaire 25 (2008), 1187-1208.

[6] E. Bonetti, G. Schimperna: Local existence to Frémond's model for damaging in elastic materials, Contin. Mech. Thermodyn. 16 (2004), 319-335.

[7] G. Bouchitté, A. Mielke, T. Roubíček: A complete-damage problem at small strains, Z. Angew. Math. Phys. 60 (2009), $205-236$.

[8] M. Bulícek, E. Feireisl, J. Málek: A Navier-Stokes-Fourier system for incompressible fluids with temperature dependent material coefficients, Nonlinear Analysis: Real World Applications 10 (2009), 992-1015.

[9] H. Brezis: Opérateurs Maximaux Monotones et Sémi-groupes de Contractions dans les Espaces de Hilbert, North-Holland Mathematics Studies, no. 5., North-Holland Publishing Co., Amsterdam-London; American Elsevier Publishing Co., Inc., New York, 1973.

[10] P. Ciarlet: Mathematical elasticity. Vol. I. Three-dimensional elasticity, Studies in Mathematics and its Applications, 20, North-Holland Publishing Co., Amsterdam, 1988.

[11] P. Colli, A. Visintin: On a class of doubly nonlinear evolution equations, Comm. Partial Differential Equations 15 (1990), $737-756$

[12] R.E. Edwards, Functional Analysis. Theory and Applications. Holt, Rinehart and Winston, New York, 1965.

[13] M. Eleuteri, E. Rocca, G. Schimperna: On a non-isothermal diffuse interface model for two-phase flows of incompressible fluids, preprint arXiv:1401.3244 (2014), 1-26, Discrete Contin. Dyn. Syst., to appear (2014).

[14] E. Feireisl: Mathematical theory of compressible, viscous, and heat conducting fluids, Comput. Math. Appl. 53 (2007), 461-490.

[15] E. Feireisl, M. Frémond, E. Rocca, G. Schimperna: A new approach to non-isothermal models for nematic liquid crystals, Arch. Rational Mech. Anal. 205 (2012), 651-672.

[16] E. Feireisl, H. Petzeltová, E. Rocca: Existence of solutions to a phase transition model with microscopic movements, Math. Methods Appl. Sci. 32 (2009), 1345-1369.

[17] E. Feireisl, E. Rocca, G. Schimperna, A. Zarnescu, Evolution of non-isothermal Landau-de Gennes nematic liquid crystals flows with singular potential, Comm. Math. Sci., 12 (2014), 317-343.

[18] E. Feireisl, E. Rocca, G. Schimperna, A. Zarnescu, Nonisothermal nematic liquid crystal flows with the Ball-Majumdar free energy, preprint arXiv:1310.8474 (2013), 1-27, WIAS Preprint No. 1865, (2013), Annali di Matematica Pura ed Applicata, doi: 10.1007/s10231-014-0419-1, to appear (2014).

[19] M. Frémond: Non-smooth thermomechanics, Springer-Verlag, Berlin, 2002.

[20] M. Frémond: Phase Change in Mechanics, Lecture Notes of the Unione Matematica Italiana 13 Springer-Verlag, Berlin, 2012.

[21] M. Frémond, B. Nedjar: Damage, gradient of damage and principle of virtual power, Internat. J. Solids Structures 33 (1996), 1083-1103.

[22] P. Germain: Cours de méchanique des milieux continus, Tome I: Théorie générale. Masson et Cie, Éditeurs, Paris, 1973.

[23] M. Grasselli, A. Miranville, R. Rossi, G. Schimperna, Analysis of the Cahn-Hilliard equation with a chemical potential dependent mobility, Comm. Partial Differential Equations 36 (2011), 1193-1238.

[24] C. Heinemann, C. Kraus: Existence results of weak solutions for Cahn-Hilliard systems coupled with elasticity and damage: Adv. Math. Sci. Appl. 21 (2011), 321-359.

[25] C. Heinemann, C. Kraus: Existence results for diffuse interface models describing phase separation and damage: European J. Appl. Math. 24 (2013), 179-211.

[26] C. Heinemann, E. Rocca: Damage processes in thermoviscoelastic materials with damage-dependent thermal expansion coefficients, preprint arXiv:1402.4177, (2014), Math. Methods Appl. Sci., to appear (2014).

[27] J. E. Marsden, T. J. R. Hughes: Mathematical foundations of elasticity, Dover Publications, Inc., New York, 1994.

[28] A.D. Ioffe: On lower semicontinuity of integral functionals, SIAM J. Control Optimization 15 (1977), 521-538.

[29] R. Klein: Laser Welding of Plastics, WILEY-VCH, 2012.

[30] D. Knees, R. Rossi, C. Zanini: A quasilinear differential inclusion for viscous and rate-independent damage systems in non-smooth domains, WIAS preprint No. 1867, (2013). 
[31] P. Krejčí, E. Rocca: Well-posedness of an extended model for water-ice phase transitions, Discrete Contin. Dyn. Syst. Ser. S 6 (2013), 439-460.

[32] P. Krejčí, E. Rocca, J. Sprekels: Liquid-solid phase transitions in a deformable container, contribution to the book "Continuous Media with Microstructure" on the occasion of Krzysztof Wilmanski's 70th birthday, Springer (2010), 285-300.

[33] J.-L. Lions, E. Magenes: Non-homogeneous boundary value problems and applications, Vol. I., Springer-Verlag, New YorkHeidelberg, 1972.

[34] E. Lorentz, S. Andrieux: Analysis of non-local models through energetic formulations, International Journal of Solids and Structures 40 (2003), 2905-2936.

[35] M. Marcus, V. Mizel: Every superposition operator mapping one Sobolev space into another is continuous, J. Funct. Anal. 33 (1979), 217-229.

[36] A. Mielke, R. Rossi, G. Savaré: Nonsmooth analysis of doubly nonlinear evolution equations, Calc. Var. Partial Differential Equations 46 (2013), 253-310.

[37] A. Mielke, T. Roubíček: Rate-independent damage processes in nonlinear elasticity Math. Models Methods Appl. Sci. 16 (2006), 177-209.

[38] A. Mielke, T. Roubíček, J. Zeman: Complete damage in elastic and viscoelastic media and its energetics, Comput. Methods Appl. Mech. Engrg. 199 (2010), 1242-1253.

[39] A. Mielke, F. Theil: On rate-independent hysteris models, Nonlinear Diff. Eq. Appl. 11 (2004), 151-189.

[40] A. Mielke, M. Thomas: Damage of nonlinearly elastic materials at small strain-existence and regularity results, ZAMM Z. Angew. Math. Mech. 90 (2010), 88-112.

[41] J. Nečas.: Les méthodes directes en théorie des équations elliptiques. Masson et Cie, Éditeurs, Paris, 1967.

[42] L. Nirenberg: On elliptic partial differential equations, Ann. Scuola Norm. Sup. Pisa (3) 13 (1959), $115-162$.

[43] R.H. Nochetto, G. Savaré, C. Verdi: A posteriori error estimates for variable timestep discretizations of nonlinear evolution equations, Comm. Pure Appl. Math. 53 (2000), 525-589.

[44] R. Rossi, G. Savaré: Gradient flows of non convex functionals in Hilbert spaces and applications, ESAIM Control Optim. Calc. Var. 12 (2006), 564-614.

[45] E. Rocca, R. Rossi: Analysis of a nonlinear degenerating PDE system for phase transitions in thermoviscoelastic materials, J. Differential Equations 245 (2008), 3327-3375.

[46] E. Rocca, R. Rossi: Global existence of strong solutions to the one-dimensional full model for phase transitions in thermoviscoelastic materials, Appl. Math. 53 (2008), 485-520.

[47] E. Rocca, R. Rossi, A degenerating PDE system for phase transitions and damage, Math. Models Methods Appl. Sci., 24 (2014), 1265-1341.

[48] T. Roubíček: Nonlinear Partial Differential Equations with Applications, Birkhäuser, Basel, 2005.

[49] T. Roubíček: Thermodynamics of rate independent processes in viscous solids at small strains, SIAM J. Math. Anal. 40 (2010), 256-297.

[50] G. Savaré: Regularity results for elliptic equations in Lipschitz domains, J. Funct. Anal. 152 (1998), $176-201$.

[51] J. Simon: Compact sets in the space $L^{p}(0, T ; B)$, Ann. Mat. Pura Appl. 146 (1987), 65-96.

[52] M. Valadier: Young measures. In: Methods of Nonconvex Analysis (Varenna, 1989), pp. 152-188. Springer, Berlin (1990).

[53] J.B. Zeld́ovich, Y.P. Raizer: Physics of shock waves and high-temperature hydrodynamic phenomena, Academic Press, New York, 1966.

[54] J. Zimmer: Global existence for a nonlinear system in thermoviscoelasticity with nonconvex energy, J. Math. Anal. Appl. 292 (2004), 589-604.

Elisabetta Rocca, Weierstrass Institute for Applied Analysis and Stochastics, Mohrenstr. 39, D-10117 Berlin, Germany, and, Dipartimento di Matematica, Università di Milano, Via Saldini 50, I-20133 Milano, Italy

E-mail address: rocca@wias-berlin.de and elisabetta.rocca@unimi.it

Riccarda Rossi, DiCATAM - Sezione di Matematica, Università di Brescia, Via Valotti 9, I-25133 Brescia, Italy

E-mail address: riccarda.rossi@unibs.it 